\title{
PERFORMANCE ANALYSIS OF VSF-OFCDM FEMTOCELLS
}

by

\section{Fatima Hussain \\ MSc. Electrical Eng. University of Engineering and Technology, Lahore, Pakistan, 2006.}

\author{
A Thesis \\ Presented to the School of Graduate Studies at \\ Ryerson University \\ in partial fulfilment of the \\ requirements for the degree of \\ Master of Applied Science \\ in the Program of Electrical and Computer Engineering
}

Toronto, Ontario, Canada, May 2012

(C)Fatima Hussain 2012 


\section{Author's Declaration}

I hereby declare that I am the sole author of this thesis.

I authorize Ryerson University to lend this thesis to other institutions or individuals for the purpose of scholarly research.

Author's Signature:

I further authorize Ryerson University to reproduce this thesis by photocopying or other means, in total or in part, at the request of other institutions or individuals for the purpose of scholarly research.

Author's Signature: 


\title{
Abstract \\ Performance Analysis of VSF-OFCDM Femtocells
}

\author{
(C)Fatima Hussain, 2012 \\ Master of Applied Science \\ Electrical and Computer Engineering \\ Ryerson University
}

In this thesis, we analyze the performance of a variable spreading factor (VSF) OFCDM employed in femtocells, with OFDM used in macrocells in a hybrid heterogenous network. Orthogonal subcarriers are assigned to macro users and for femtocell users, non-contiguous subcarrier grouping is employed. We derive the analytic expression of the BER for uplink VSF-OFCDM femto and OFDM macro users for the case of maximal ratio combining receiver. We evaluate the performance of femto/macro users in VSF-OFCDM system through numerical and Monte Carlo simulation studies. Improvement in BER of the femtocell users is also noted. The relationship between the femto spreading factor and femto/macro BER is analyzed. We present, the relationship between the channel load and optimum spreading factor employed by femtocell users for the energy efficient performance of macro users. Femto wall penetration loss, that is the important parameter to evaluate the femto performance, is also taken into account. Also, effect of femto wall penetration on macro BER is evaluated for various spreading factors.

Following our study, we find that interference-limited system favors increased time spreading especially when number of subcarriers is limited and noise-limited system favors increased frequency domain spreading. When large number of subcarriers are available, optimum spreading (from macro perspective) favors increased frequency domain spreading regardless of the femto-macro loads, or whether operating environment is noise or interference limited. Once the optimum spreading factor is determined, increase or decrease in the femto Eb/No does not matter. Also, femto wall penetration factor not only effects the femto BER directly, but also reduce the potential interference faced by macro user equipment (UEs). As a result macro BER is improved, but the choice of optimal spreading factor for macro UEs remain unaffected with the variation in femto wall penetration loss. 


\section{Acknowledgement}

I would like to express my sincere gratitude to my supervisor, Prof. Alagan Anpalagan for his continuous encouragement and support. He was always generous with his invaluable time and always motivating me. It was a great privilege to work with him. I look forward to continue working with him in future.

I can never find the words to thank my husband, Javaid Talib, who was amazingly patient and understanding and who helped me through the most difficult times of my life. He believed in me more than I do. He was always supportive and offered me his valuable help whenever I needed it.

My special thanks goes to my mother, may she rests in peace, who is always a great source of love and motivation, I could never thank her enough for everything she has done for me. I would also like to thank my father in-law for his prayers and encouragement.

Last but not the least, I would like to thank my son, Hamza, who is very understanding child and cheers me up when I am down. He always gives me a motivation to be a super-mom, that he thinks I am. 


\section{Contents}

1 Introduction $\quad 1$

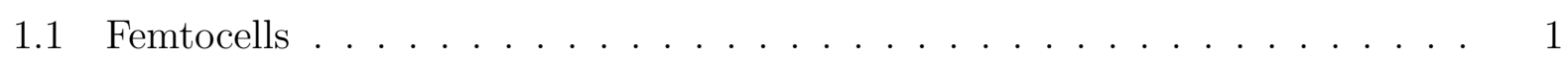

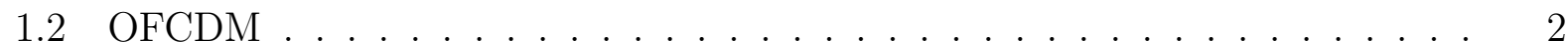

1.3 Thesis Contribution . . . . . . . . . . . . . . . . . . 4

1.4 Thesis Organization . . . . . . . . . . . . . . . . 5

2 Basics of Femtocells and OFCDM $\quad 6$

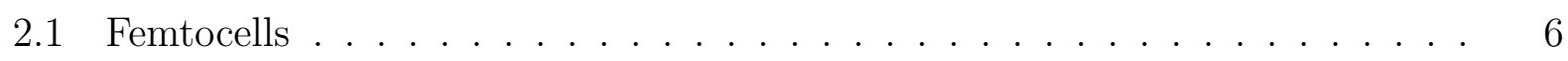

2.2 Multicarrier CDMA Systems . . . . . . . . . . . . . . . . . . . . . 10

2.2.1 Orthogonal Frequency Division Multiplexing (OFDM) . . . . . . . 10

2.2.2 Code Division Multiple Access (CDMA) . . . . . . . . . . . . 11

2.2.3 Orthogonal Frequency Code Division Multiplexing (OFCDM) . . . . 12

2.3 OFCDM and Femtocells . . . . . . . . . . . . . . . . . . 13

3 Literature Survey $\quad 16$

3.1 Technical Challenges . . . . . . . . . . . . . . . . 16

3.1.1 Resource Allocation and Management . . . . . . . . . . . . . . . . 17

3.1.2 Multiple Access Method . . . . . . . . . . . . . . . . . . . 18

3.1.3 Interference Avoidance and Management . . . . . . . . . . . . . . . 19

3.2 Multicarrier Femtocell System . . . . . . . . . . . . . . . . . . . . . . . . 20

3.2 .1 OFDMA Femtocells . . . . . . . . . . . . . . . . . . . 21 
3.2 .2 CDMA and MC-CDMA Femtocells . . . . . . . . . . . . . . . 21

3.2.3 Two Dimentional (2D) Spreading . . . . . . . . . . . . . 23

3.3 Comparison of Multicarrier Femtocell Systems . . . . . . . . . . . . . . . 24

4 Transceiver Model for OFDM Macro and VSF-OFCDM Femto Cells 27

4.1 OFDM System Model for Macro Users . . . . . . . . . . . . . . . . 27

4.1.1 Orthogonal Channel Assignment . . . . . . . . . . . . . . . . . 29

4.1 .2 OFDM Transmitter . . . . . . . . . . . . . . . . . . 29

4.1 .3 Outdoor Channel Model . . . . . . . . . . . . . . . . 30

4.1 .4 OFDM Receiver . . . . . . . . . . . . . . . . . 31

4.2 VSF-OFCDM System Model for Femto Users . . . . . . . . . . . . . . . . 31

4.2.1 Channel Grouping and Assignment . . . . . . . . . . . . . . . 32

4.2 .2 VSF-OFCDM Transmitter . . . . . . . . . . . . . . . . 33

4.2 .3 Indoor Channel Model . . . . . . . . . . . . . . . . . . 34

4.2 .4 VSF-OFCDM Receiver . . . . . . . . . . . . . 35

5 BER Performance Analysis $\quad 38$

5.1 Hybrid Network Scenario . . . . . . . . . . . . . . . . 38

5.2 System Parameters . . . . . . . . . . . . . . . . . . 40

5.3 Channel Models . . . . . . . . . . . . . . . . . . . 41

5.4 Uplink Interference Analysis . . . . . . . . . . . . . . . . . . . 42

5.5 BER Calculation . . . . . . . . . . . . . . . . . . . 44

5.5.1 SINR of Femto Users . . . . . . . . . . . . . . . . 44

5.5 .2 SINR of Macro Users . . . . . . . . . . . . . . . . . 47

6 Results and Discussion $\quad 49$

6.1 Simulation Setup and Parameters . . . . . . . . . . . . . . . . 49

6.2 Femto BER $\sim$ Femto Eb/No . . . . . . . . . . . . . . 50

6.2.1 Interference-Limited Case . . . . . . . . . . . . . 50 
6.2 .2 Noise-Limited Case . . . . . . . . . . . . . . . . . . . . . . . . 52

6.3 Macro BER $\sim$ Femto Eb/No . . . . . . . . . . . . . . . 53

6.3.1 Interference-Limited Case . . . . . . . . . . . . . . . 53

6.3 .2 Noise-Limited Case . . . . . . . . . . . . . . . . . . . . 54

6.4 Mcaro BER $\sim$ Macro Eb/No . . . . . . . . . . . . . . . . . 54

6.4.1 Interference-Limited Case . . . . . . . . . . . . . . . . 55

6.4.2 Noise-Limited Case . . . . . . . . . . . . . . . . . . . . 56

6.5 Optimal Spreading Factor . . . . . . . . . . . . . . . 58

6.5.1 Interference-Limited Case . . . . . . . . . . . . . . . . . . . 59

6.5.2 Noise-Limited Case . . . . . . . . . . . . . . . . . . . . . . . . . 59

6.5.3 Moderate Users Case . . . . . . . . . . . . . . . . . 62

6.6 Femto Wall Penetration . . . . . . . . . . . . . . . . . . . 64

6.6 .1 Femto BER ........................ 65

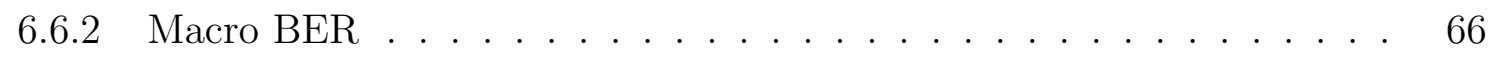

6.7 Optimal Spreading at Various Channel Loads . . . . . . . . . . . . . . . . . 70

7 Conclusions and Future Work $\quad 73$

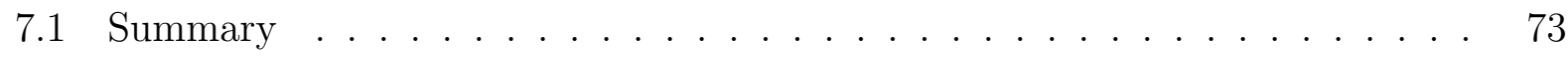

7.2 Future Research . . . . . . . . . . . . . . . . . . . . 75 


\section{List of Figures}

2.1 Femtocells Deployment Scenarios $[1] \ldots \ldots \ldots$

2.2 Cellular Zones $[1] \ldots \ldots \ldots \ldots$

2.3 Transmitter/Receiver of OFCDM System . . . . . . . . . . . . . . . . 14

4.1 Transmitter/Receiver of OFDM System . . . . . . . . . . . . . . 28

4.2 Subcarrier grouping with 3 subcarrier groups. . . . . . . . . . . . . . . . 32

4.3 Transmitter of OFCDM System . . . . . . . . . . . . . . . . . . 34

4.4 Receiver of OFCDM System . . . . . . . . . . . . . . . . 36

5.1 Femto/Macro Network Scenario . . . . . . . . . . . . . . . . . . . 39

5.2 Coverage area of Macro and Femto Cells, $R_{f} \ll R_{m} \ldots \ldots \ldots$. . . . . . 40

5.3 Interferences among Femto and Macro Users. . . . . . . . . . . . . . . . . . . 43

6.1 Femto BER in Interference Limited Case $(64 \mathrm{~F}-64 \mathrm{M}) \ldots \ldots$. . . . . . . . . . 51

6.2 Femto BER in Noise Limited Case $(16 \mathrm{~F}-16 \mathrm{M}) \ldots \ldots \ldots$

6.3 Macro BER vs. Femto Eb/No $(64 \mathrm{M}-64 \mathrm{~F}) \ldots \ldots \ldots \ldots \ldots$

6.4 Macro BER vs. Femto Eb/No $(16 \mathrm{M}-16 \mathrm{~F}) \ldots \ldots \ldots \ldots$

6.5 Macro BER in Interference Limited Case $(64 \mathrm{~F}-64 \mathrm{M}) \ldots \ldots$. . . . . . . . . 56

6.6 Macro BER in Noise Limited Case $(16 \mathrm{~F}-16 \mathrm{M}) \ldots \ldots$. . . . . . . . . 57

6.7 Macro BER vs. Femto Spreading in Interference Limited Case (64F-64M) . . 58

6.8 Macro BER vs. Femto Spreading in Noise Limited Case (16F-16M) . . . . . 60

6.9 Macro BER vs. Femto Spreading $(64 \mathrm{~F}-16 \mathrm{M}) \ldots \ldots \ldots$. . . . . . . . . 61 
6.10 Macro BER vs. Femto Spreading $(32 \mathrm{~F}-16 \mathrm{M}) \ldots \ldots \ldots$

6.11 Macro BER vs. Femto Spreading $(32 \mathrm{~F}-64 \mathrm{M}) \ldots \ldots . \ldots . \ldots . \ldots$

6.12 Femto BER in Interference Limited Case (64F-64M) for Various $W_{f} \ldots \ldots$

6.13 Femto BER in Noise Limited Case $(16 \mathrm{~F}-16 \mathrm{M})$ for Various $W_{f} \ldots \ldots$. . . . 65

6.14 Macro BER vs. Femto Spreading in Interference Limited Case (64F-64M) for

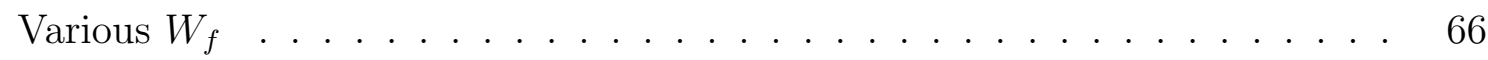

6.15 Macro BER vs. Femto Spreading in Noise Limited Case (16F-16M) for Various

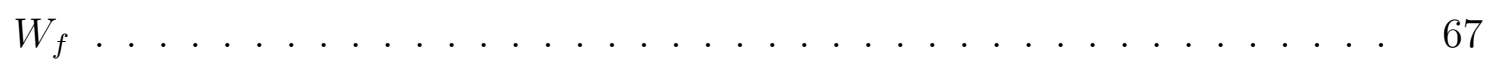

6.16 Macro BER vs. Femto Spreading $(32 \mathrm{~F}-64 \mathrm{M})$ for Various $W_{f} \ldots \ldots$

6.17 Macro BER vs. Femto Spreading $(64 \mathrm{~F}-16 \mathrm{M})$ for Various $W_{f} \ldots \ldots$. . . . . 69 


\section{List of Tables}

2.1 Comparison Among Different Indoor Coverage Techniques [2]. . . . . . . . . 9

3.1 Comparison of Various Multicarrier Techniques for Femtocells . . . . . . . . 25

6.1 Simulation Parameters . . . . . . . . . . . . . . . . . . 50

6.2 Optimum Spreading Factor for Various Channel Loads for 64 Subcarriers . . 70

6.3 Optimum Spreading Factor for Various Channel Loads for 128 Subcarriers . 70

6.4 Optimum Spreading Factor for Various Channel Loads for 256 Subcarriers . 71 


\title{
List of Abbreviations
}

\author{
4G Fourth Generation \\ AWGN Additive White Gaussian Noise \\ B3G Beyond Third Generation \\ BER Bit Error Rate \\ BTS Base Transceiver Station \\ BPSK Binary Phase Shift Keying \\ CDMA Code Division Multiple Access \\ CP Cyclic Prefix \\ DSL Digital Subscriber Line \\ DFT Discrete Fourier Transform \\ FDMA Frequency Division Multiple Access \\ FEC Forward Error Correction \\ FFT Fast Fourier Transform \\ FAP Femtocell Access Point \\ FCC Federal Communications Commission \\ GPRS General Packet Radio Service \\ GPS Global Positioning System \\ GSM Global System for Mobile communication \\ HBS Home Base Station \\ HCS Hierarchical Cell Structure \\ HeNB Home eNodeB \\ HLR Home Location Register \\ HNB Home NodeB \\ ICI Intercarrier Interference \\ IFFT Inverse Fast Fourier Transform \\ i.i.d independent identically distributed




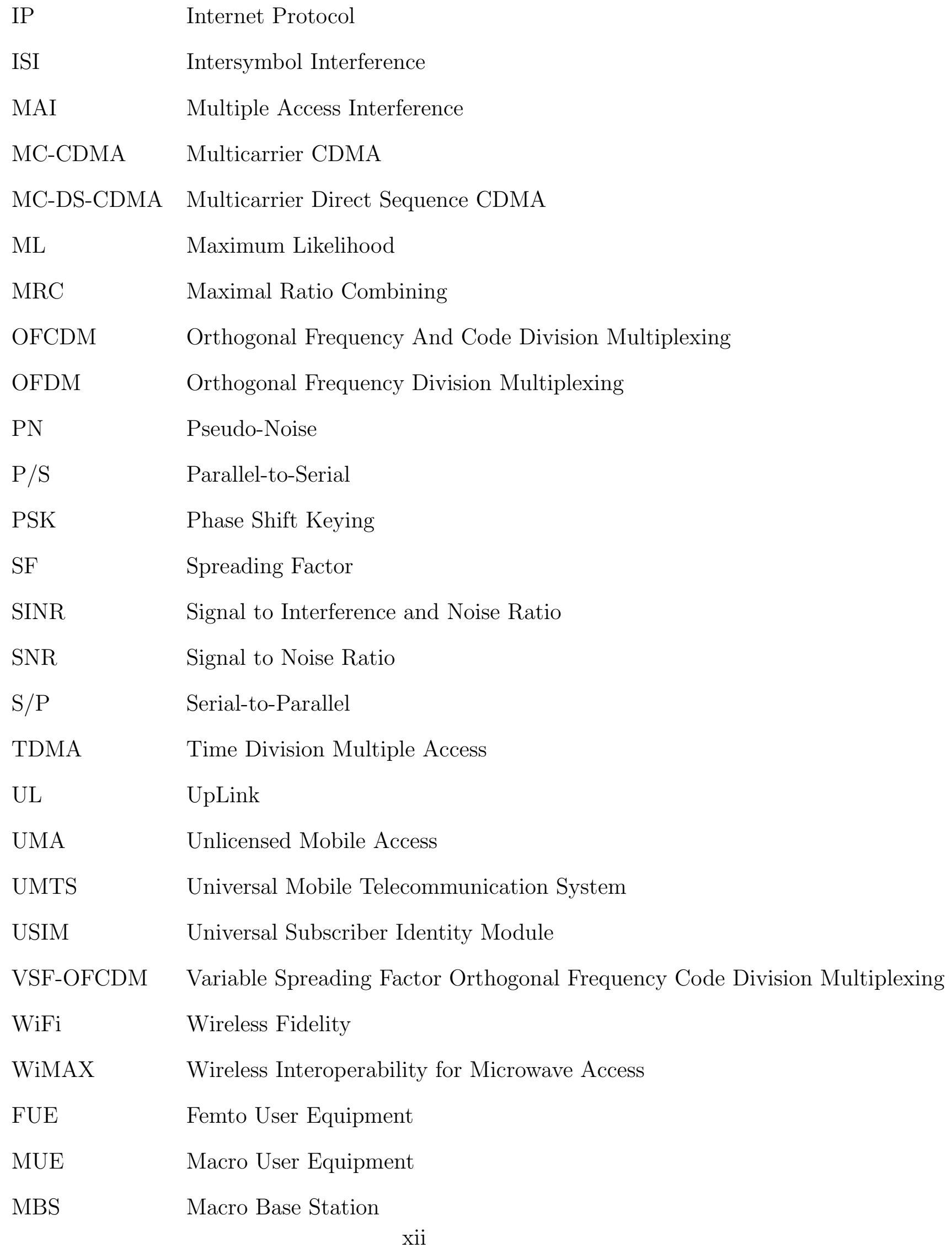




\section{Chapter 1}

\section{Introduction}

In cellular wireless networks, it is estimated that $2 / 3$ of voice calls and over 90 percent of data services occur indoors. Hence, it is extremely important for cellular operators to provide good indoor coverage for not only voice but also for video and high speed data services, which are becoming increasingly important [1]. Indoor solutions such as picocells become an attractive and viable business proposition in hotspots such as large business centers, office buildings and shopping malls. These indoor systems are deployed by the operators. The indoor solutions will improve in-building coverage, offload traffic from outdoor macrocells, enhance service quality and facilitate high data rate services due to the improved performance of shorter and perhaps direct radio links. Even though the above mentioned indoor solutions are more cost effective than using outdoor macrocells to provide indoor coverage for voice and high speed data services, such solutions are still too expensive. Recently, the development of femtocells provides a good opportunity for low cost indoor solutions; unlike picocells, femtocells are deployed by end users.

\section{$1.1 \quad$ Femtocells}

Femtocells, also known as "HBS" (home base stations), are cellular network access points that connect standard mobile devices to a mobile operator network using residential DSL, 
cable broadband connections, optical fibres or wireless link [2].

The concept of home base station was first studied by Bell Labs of Alcatel-Lucent in 1999. In 2002, Motorola announced the first "3G-based" home base station product. However, it was not until 2005 that the home base station concept started to gain a wider acceptance. In 2006, femtocell as a term was coined. In July 2007, the Femto Forum was founded to promote femtocell standardization and deployment worldwide. As of December 2008, the forum includes over 100 telecom hardware and software vendors, mobile operators, content providers and start-ups [1].

The femtocell unit incorporates the functionality of a typical base station and looks like a WiFi access point. However, it also contains RNC (Radio Network Controller) and all the core network elements. Thus, it does not require a cellular core network, requiring only a data connection to the DSL or cable to the Internet, through which it is then connected to the mobile operators core network. In this thesis, we use femtocell access point (FAP) to stand for the femtocell unit that contains base station and core network functionalities, and use femtocell to refer to the service area covered by the FAP. The main drive of FAP is to provide high data rate services for the indoor users. These are self-deployed by users rather than the operators. FAP can automatically configure itself and this is key to the successful deployment of femtocells. We will analyze the performance of OFCDM femtocell users utilizing the shared spectrum with macrocell users that employ OFDM technology.

\subsection{OFCDM}

The Fourth Generation (4G) of wireless communications systems have sparked much interest in the recent years. Higher data rates, an increase of capacity in the radio link and seamless mobility across heterogeneous access networks are some of the most recognized traits of $4 \mathrm{G}$ systems [3]. One of the strongest aspects of $4 \mathrm{G}$ systems involves the merger of cellular and wireless technologies, rather than being an entirely new standard [4]. To achieve 4G 
performance, new technologies related to multiple access, adaptive modulation and coding must be developed to be used in conjunction with the previous technologies.

$4 \mathrm{G}$ systems are likely to employ improved multiple access schemes to achieve the expected throughput of $100 \mathrm{Mbps}$ and $20 \mathrm{Mbps}$ in the downlink and uplink of a cellular system, respectively [5]. 4G heavily relies on orthogonal frequency division multiplexing (OFDM) based techniques [6]. OFDM uses parallel transmission of data symbols on orthogonal subcarriers to reduce the transmission rate and increase the symbol duration, thus enabling data transmission with very low inter-symbol interference (ISI). Some variations of OFDM that employ spread spectrum technology allowing multiple user access are Multicarrier Direct Sequence CDMA (MC-DS-CDMA) [7], Multicarrier CDMA (MC-CDMA) and Orthogonal Frequency and Code Division Multiplexing (OFCDM) [8].

OFCDM is based on MC-CDMA and has been studied as the best candidate for 4G [9]. In OFCDM, data streams are segmented into multiple substreams and spread over successive symbols in time domain and over successive subcarriers in frequency domain, thus combining time and frequency domain spreading. The main advantage of OFCDM is the diversity gained as symbols are replicated in time and across several frequency subcarriers.

The demand for high data rates in future wireless communications has made it essential to investigate methods of achieving high spectral efficiency since the spectrum is considered a valuable commodity. We will be utilizing OFCDM for the spectrum assignment in femtocells due to its robustness to channel variations and provision of variable spreading factor. Femtocells will be sharing the spectrum with the existing macrocell users under various spreading factors and different channel loads. For traceability of analysis, we assume that OFCDM is employed by femtocell users and OFDM is employed by macrocell users, in uplink communication.

Many researchers in [10-13] have investigated the usefulness of multicarrier schemes in macro/femto hybrid network. CDMA, OFDMA and MC-CDMA have been utilized for the analysis of the relationship between femto and macro networks for various channel conditions, 
channel loads and transmit power. However, the authors do not take into account the consideration for the relationship between optimum/unique spreading factor and femto/macro user density. Also, to the best of author's knowledge, this is the first study where macro users are utilizing OFDM and femto users are using $\mathrm{OFCDM}^{1}$ for their spectrum allocation. In previous research, both the systems were utilizing the same technology such as, CDMA or OFDM for their spectrum allocation. Our research is more realistic in a sense that OFDM system is widely used in cellular network thus providing an ease of deployment of femto/macro hybrid network.

\subsection{Thesis Contribution}

The key contributions of this thesis can be summarized in the following points. We

- derive a closed form expression for the total SINR (and BER) for femtocell and macrocell users in a VSF-OFCDM system in the presence of the different types of interferences, spreading factors and load conditions. We also evaluate the variation in BER degradation of a macrocell users under these conditions.

- propose to use a subcarrier grouping strategy, that was earlier proposed for macrocell networks, to maximize gains from frequency diversity in the hybrid macro/femto network.

- evaluate the performance of femtocell and macrocell users in terms of the spectral efficiency in the VSF-OFCDM system through numerical and Monte Carlo simulation studies. Improvement in spectral efficiency of the femtocell users is noted.

- present the relationship, in the form of a table, between the channel load and optimum spreading factor employed by femtocell users for the energy efficient performance of

\footnotetext{
${ }^{1}$ Note that one could employ OFCDM in macrocells as well in general; however, it will mask the effect of OFCDM in femtocells, and lead to un-traceability in the analysis. Hence, we assumed OFCDM for femtocells.
} 
macro users.

- analyze the relationship between the femto wall penetration loss and femto BER at various channel loads. Also, effect of femto wall penetration on macro BER is evaluated for various spreading factors.

\subsection{Thesis Organization}

This thesis is organized as follows:

In chapter 2, detail introduction of femtocells is given. We discuss the need, importance and benefits of this new technology. This also includes a description of two dimensionally spread OFCDM system along with its usage in femtocells.

In chapter 3, various technical challenges faced in efficient deployment of femtocells are discussed. Also contributions of different researchers to address these issues are presented. Related work in the area of multicarrier femtocells so far done, is also presented.

In chapter 4, we outline in details the hybrid system model with femto and macro cells used in this research. We also discuss the subcarrier grouping strategy being used in our work. This includes a description of the transmitter and receiver of an OFCDM system. Afterwards, the system model for the VSF-OFCDM femtocell is also presented.

In chapter 5, we analyze the BER performance of co-channel deployment of two tier femto/macro network under different spreading factors and different channel loads. We also, derive the SINR expression for femto and macro users. Afterwards, simulation parameters for both types of users are given.

In chapter 6, simulation results and its theoretical explanation are presented. Various plots and their results in the form of table are provided to support the argument.

In chapter 7, we conclude the thesis with the interpretation of important results. Also, possible future work is presented in this chapter. 


\section{Chapter 2}

\section{Basics of Femtocells and OFCDM}

The growing number of cellular network users raises issues about coverage extension in some areas such as rural zones, indoors or underground locations. Mobile network operators now offer femtocell service plans for both voice and data to residential and commercial customers. FAPs become popular with every passing day. In this chapter, we will discuss femtocells and its applications, and provide a comparison among various indoor coverage techniques. Afterwards, a brief introduction of OFDM and CDMA systems is given. OFCDM and its benefits along with its utility in femtocells is presented at the end.

\section{$2.1 \quad$ Femtocells}

Femtocells are smaller scale cellular access points typically for indoor usage and require very little upfront cost. This cellular gateway communicates with the cellular network over a broadband connection such as digital subscriber line (DSL), cable modem, or a separate radio frequency $(\mathrm{RF})$ backhaul channel. Conventional approaches require dual-mode handsets to deliver both in-home and mobile services, but in-home femtocell deployment promises fixed mobile convergence with existing handsets [2]. It proves to be a valuable solution for locations where conventional cellular tower are not installed or locations covered with weak wireless signal. 


\section{Salient Features}

Some of the key features of femtocells include:

- Femtocells can provide significant power saving to UE. The path loss to indoor FAPs is much smaller than that to the outdoor MBS, and so is the required transmitting power from UE to the FAP. As switching of FAP is only on demand, its use is greener than macrocells [14]. Also traditional base station consumes far more power than that is used for transmitting and receiving signals [15]. This is caused by a number of factors: first, the efficiency of the amplifiers is very low in linear region and techniques used in 3G and beyond systems require linear amplification; second, a base station requires an air-conditioning system in order to keep running at atmospheric temperature; third, a backup system is also needed to account for loss of power supply. This base station power consumption problem leads to a high demand on the so-called green communications systems or "green radio".

- Femtocells operate in licensed spectrum and reuse the technologies used for wide area cellular networks. These are compatible with UEs designed for macrocells.

- Growing demand for higher and higher data rates makes it difficult for macrocells to fulfil the requirements alone due to its high penetration losses. Therefore, requirement of high data rate services is the key driver for femtocells [16].

Figure 2.1 shows various femtocells deployment scenarios [1]. In this figure, we can see various FAPs deployed within the coverage area of several MBSs. It depicts a single FAP in home, networked FAPs in an apartment building and mobile femto in a moving vehicle. In our work we have assumed the in-home FAPs uniformly deployed in the coverage area of an MBS. 


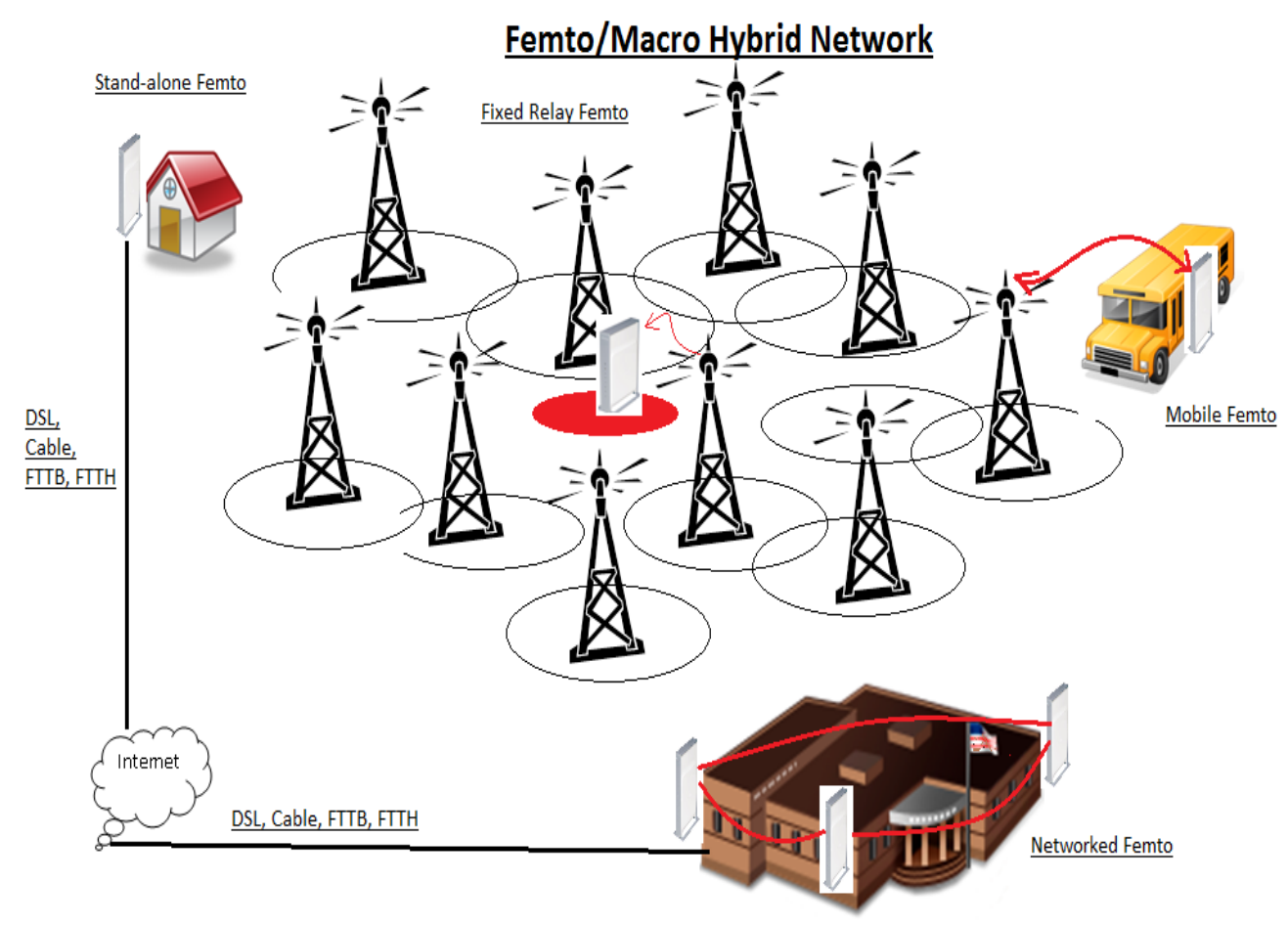

Figure 2.1: Femtocells Deployment Scenarios [1]

\section{Applications}

Femtocells could be deployed in different kinds of scenarios. The first market is in the home where multiple mobile phone users want to access the Internet. The second market is office environments, in which, buildings are covered by a set of femtocells. In rural environments, due to insufficient macrocell coverage, femtocells can be a suitable approach. In urban environments, femtocell is a good approach for covering dense building areas, where the customers, apartments are rarely in direct view of a macrocell, and where the losses due to the other buildings cause poor indoor coverage [17]. As for femtocells the indoor coverage will be maximum, some new data intensive services can be experienced by the customers. As described in [2], the new services can be divided into two kinds of applications: Femtozone Services and Connected Home Services. Femtozone services are web/voice automatic services that are activated when the customer enters/leaves the range of their femtocells. In Connected Home services the phone accesses the LAN via the femtocell and acts as controller 
to manage all connected equipment inside the house, for example, controlling a TV, HiFi or uploading music to PC from mobile phone.

These services are thus made possible via evolving new technologies, and there are currently few propositions in this area [17]. However, it is important to note that the success of femtocell technology depends mainly on these new kinds of applications. Such services could attract more customers and also help to diversify the role of the mobile, thus making more revenue for the operators.

\section{Comparison of Indoor Coverage Techniques}

The indoor coverage can be extended with different methods, such as increasing the outdoor signal power, or adding more cells, or re-transmitting the outdoor signal inside the building by using repeaters. However, these solutions are not desired because they do not really optimize the indoor coverage, but only extend the effects of outdoor coverage inside the buildings.

To directly increase the indoor coverage, there are some efficient solutions such as installing base stations directly inside the buildings. Also, radiating cable is a good approach for long narrow environments such as tunnels, but is very challenging and expensive inside the large buildings [1]. While inside home, small base stations such as picocells and femtocells are efficient solutions as shown in Figure 2.2. If their positions are judiciously chosen, the indoor signal power can be efficiently improved and will increase the potential capacity of the system. In Table 2.1, a summary of the main ideas of the previously described indoor coverage techniques is presented [2].

\begin{tabular}{|c|c|c|c|c|c|}
\hline Factors & Macro/Micro & Repeater & DAS & Cable & Pico/Femto \\
\hline \hline Price & Expensive & Convenient & Convenient & Convenient & Cheap \\
\hline Installation & Expensive & Difficult & Easy & Difficult & Very Easy \\
\hline Power Used & High & Low & Low & Low & Very Low \\
\hline Indoor Coverage & Bad & Acceptable & Good & Good & Good \\
\hline
\end{tabular}

Table 2.1: Comparison Among Different Indoor Coverage Techniques [2]. 


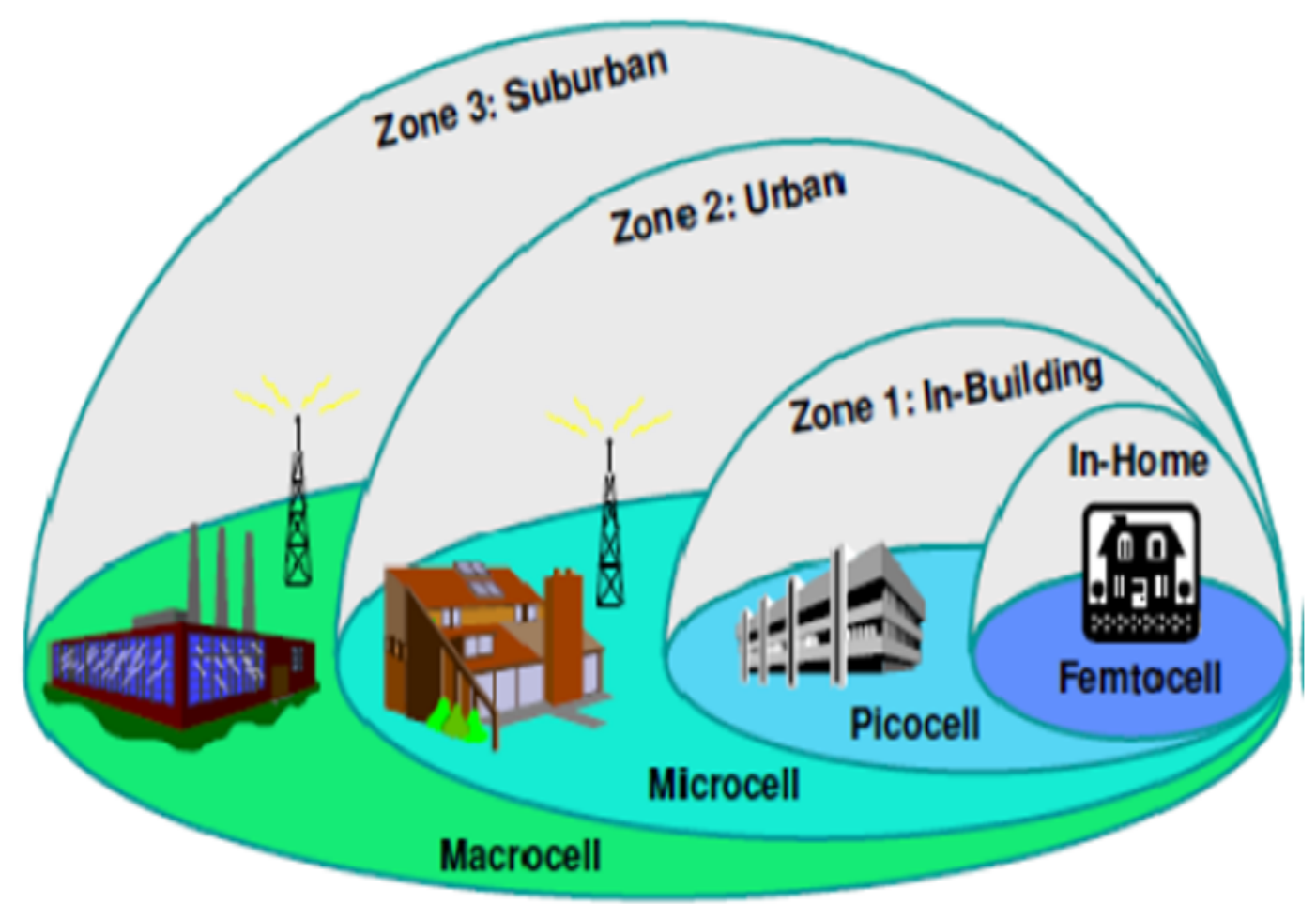

Figure 2.2: Cellular Zones [1]

\subsection{Multicarrier CDMA Systems}

In this section, we will first discuss OFDM, CDMA and multi-carrier CDMA. Afterwards, OFCDM, its benefits and its utility in femtocells are presented.

\subsubsection{Orthogonal Frequency Division Multiplexing (OFDM)}

OFDM is a special form of multicarrier transmission. The basic principle of OFDM is to split a high-rate data stream into a number of lower rate streams that are modulated and transmitted simultaneously over different subcarriers centered at respective bands. The use of parallel transmission effectively increases the symbol duration and considerably reduces ISI [18]. By choosing the subcarrier's bandwidth to be less than the coherence bandwidth of the channel, it experiences almost flat fading which makes equalization very simple. Orthogonal 
subcarriers are used to ensure proper retrieval of data at the receiver end and to minimize interference. The orthogonality of the subcarriers allows the spectra of individual subcarriers to overlap with each other without any significant interference which allows for more efficient use of the bandwidth. Also, OFDM is very robust to narrow band interferences because such interference can only affect a small percentage of the subcarriers [19].

Main advantages of multicarrier OFDM can be sumarized as:

- Robustness against multipath

- Efficient implementation through FFT blocks

- Utilization of frequency diversity for multiple user access

- High spectral efficiency

- Robustness against narrowband interference from nearby terminals

\subsubsection{Code Division Multiple Access (CDMA)}

Code division multiple access is a spread spectrum (SS) communication technique for the transmission of digital information. At the transmitter, the message signal is spread over a large bandwidth using a PseudoNoise $(\mathrm{PN})$ code. At the receiver, the identical PN code is utilized to restore the original message signal. In SS systems, the PN sequence is only known by the intended user, and the SS signal appears as wideband noise to un-intended receivers. As a result, privacy is inherent in SS systems. Also, spread spectrum systems benefit from a low power spectral density, which makes these systems very difficult to detect by an unintended user. In addition, the spectral properties of the PN codes make signals very resistant to intentional jammers [20].

In early 90's these systems were introduced into the market by Qualcomm in the form of Direct-Sequence Code Divion Multiple Access (DS-CDMA) [21]. It provides multiple access 
by assigning PN codes with low cross correlation to users. These systems have proved their significance in cellular networks.

CDMA has been considered as a strong candidate to support multimedia mobile services because it has the ability to cope up with the asynchronous nature of the multimedia traffic and can provide higher capacity as opposed to the conventional access schemes such as, Time Division Multiple Access (TDMA) or Frequency Division Multiple Access (FDMA). By employing Rake receivers, CDMA systems can coherently combine the multipath components due to the hostile frequency selective channel. The processing gain due to spreading provides robustness to the multiuser interference. The use of conventional CDMA does not seem to be realistic when the data rates become very high due to severe ISI and the difficulty in synchronizing a fast sequence. Techniques for reducing the symbol and chip rate are essential in this case. Combining multicarrier OFDM transmissions with CDMA spreading allows us to use the available spectrum in an efficient way and retains the many advantages of a CDMA system as explained in the following. The use of multicarrier CDMA not only mitigates the effect of ISI but also exploits the multipath effect [22].

\subsubsection{Orthogonal Frequency Code Division Multiplexing (OFCDM)}

Combination of OFDM-CDMA is a useful technique for $4 \mathrm{G}$ systems featuring variable data rates and reliable communication systems. Prasad and Hara [22] compared various methods of combining the two techniques, based on spreading operation that takes place either in time or frequency domain. The OFDM with spreading in time or frequency domain only is known as Multicarrier DS-CDMA (MC-DS-CDMA) and Multicarrier CDMA (MC-CDMA), respectively. In MC-DS-CDMA, the data symbols are serial-to-parallel converted and spread using a spreading sequence in the time domain which provides flexible data rates and reduces the multiple access interference (MAI). In MC-CDMA the individual data symbols are spread using a spreading code in the frequency domain. The main advantage of MC-CDMA is

the frequency diversity gained by transmitting over subchannels experiencing independent 
fading.

The multicarrier system with two dimensional spreading was proposed in $[8,23]$ for cellular systems, in which spreading is employed in both time and frequency domains simultaneously. This is known as Orthogonal Frequency and Code Division Multiplexing (OFCDM). In OFCDM, data streams are segmented into multiple substreams and spread by the spreading sequences, which can be the combination of an orthogonal short channelization code, short Walsh-Hadamard codes and long PN codes.

Each chip of the resultant sequence is allocated to the successive OFCDM symbols in the time domain (called time domain spreading) and to the successive subchannels in the frequency domain (called frequency domain spreading).

Figure 2.3 is the block diagram of OFCDM and defines each block used for the signal generation and reception.

\section{Variable Spreading Factor OFCDM}

The Variable Spreading Factor (VSF)-OFCDM, which changes the spreading factor both in time and frequency domain, was proposed in $[9,24,25]$ to study the impact of spreading variations on the spectral efficiency and BER improvement. In OFCDM, the total spreading factor $S F$, is expressed as $S F=S F_{\text {time }} \times S F_{\text {freq }}$, where $S F_{\text {time }}$ and $S F_{\text {freq }}$ represent the spreading factors in the time and frequency domain respectively. The time and frequency domain spreading factors can be changed flexibly to provide variable spreading factor in order for the system to work adaptively in different cell environments and channel conditions [21].

\section{$2.3 \quad$ OFCDM and Femtocells}

Numerous researchers have presented results to examine spreading factors that are favorable under different operating conditions for OFCDM systems [26-28]. Their findings reveal that a high frequency domain spreading factor is superior under low channel loads. This occurs 


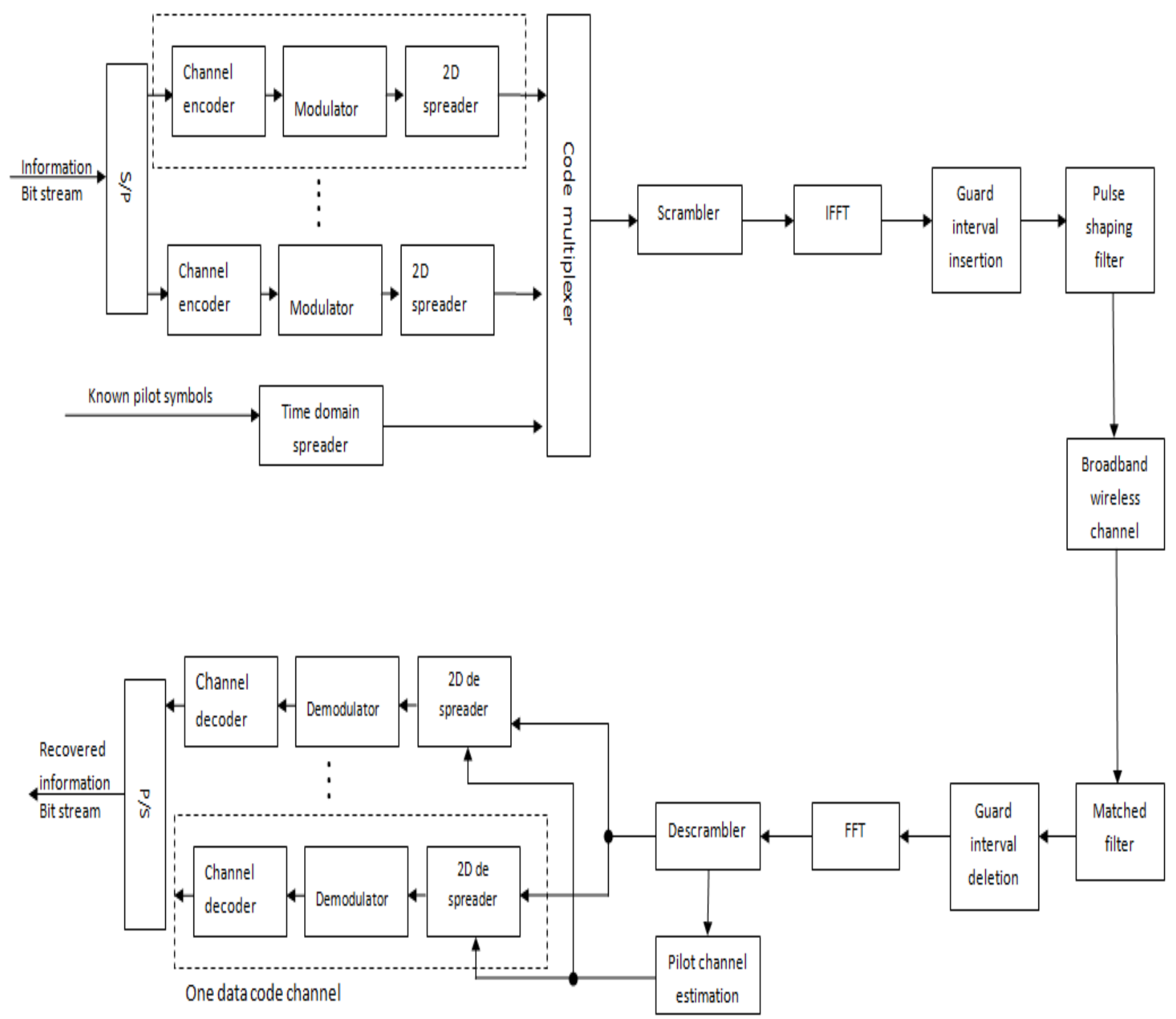

Figure 2.3: Transmitter/Receiver of OFCDM System

because frequency diversity is achieved, while multiple access interference (MAI) remains small because only few users access the channel simultaneously. If there is a requirement to support a large capacity of users, however, time domain spreading should be prioritized to minimize MAI. For specific channel conditions and users density, particular time and frequency domain spreading factors that are most favorable, are required. 
Keeping in mind the benefits of OFCDM, we are utilizing it in the spectrum assignment of femtocells. It will incorporate the characteristics of spreading in both the dimensions for the co-channel existence of both femto/macro users. Spreading in frequency will enhance the overall spectrum efficeincy and femto users will be sharing the same licenced spectrum without disturbing the functionality of existing macro users. Spreading in time will also enhance the overall system capacity along with reduction in MAI among the femto and macro users. Moreover, the provision of varaible spreading factor both in time and frequency, facilitates the adaptability of spreading according to the cell structure variations, femto/macro hybrid system load and radio link conditions such as delay spread.

In the next chapter, we will discuss various challenges faced by developers and system providers in the efficient deployment of femtocells. The advantages of multicarrier femtocell system and the comparison of its variations are also presented. This includes a description of OFDMA femtocell systems, CDMA femtocell systems and OFCDM femtocell systems. 


\section{Chapter 3}

\section{Literature Survey}

A large amount of phone calls, approximately 90\%, are placed indoor [2] and macrocells are not very efficient for complete indoor coverage due to high penetration losses. To fill in coverage holes, femtocells in various forms are emerging as a promising technology. Femtocells will enhance indoor coverage, deliver high bandwidths, offer new services, and offload traffic from existing networks [2]. However, the deployment of a new femtocell layer may have an undesired impact on the performance of the existing macrocell layer. The allocation of spectrum resources and the avoidance of electromagnetic interference are some of the important challenges that operators face before femtocells become widely deployed. In this chapter, an overview of technical challenges it's facing is given. Afterwards, use of multicarrier techniques is presented, as a solution, to combat the interference issues.

\subsection{Technical Challenges}

Many researchers have highlighted various technical challenges such as resource allocation, timing/synchronization, interference management, access management, mobility management and above all securely bridging the femtocell with the operator's network. The most notable areas of challenge from the interference point of view are discussed next: 


\subsubsection{Resource Allocation and Management}

Radio resource management is an efficient tool used in cellular systems to mitigate interference. Broadly speaking, two types of channel assignment are possible:

\section{Co-Channel Assignment}

Co-channel assignment can be either centralized or distributed. In a centralized approach, a central entity is in-charge of intelligently controlling each cell which subchannel is to use. This entity collects information from the femtocells and their users, and use them to find an optimal or good solution within a short period of time. But since the number of femtocells is large, this makes resource optimization problem too complex. While in a distributed approach, each cell manages its own subchannels thus, it does more of self-organization. It can be either co-operative or non-cooperative distributive assignment [2]:

- In a non-cooperative approach, access to the subchannels is opportunistic. Each FAP plans its subchannels in a way to maximize the throughput and quality of service (QoS) for its users. Furthermore, it is independent of the effects its allocation might cause to the neighboring cells.

- In a co-operative approach, each FAP gathers information about neighboring femtocells and performs its allocation taking into account the effects it would cause to neighbors. It is very beneficial both in terms of resource management and interference reduction but has the disadvantage of requiring additional overhead of sensing mechanism to gather information about neighboring femto and macro cells.

\section{Orthogonal Channel Assignment}

Orthogonal channel assignment divides the licensed spectrum into two parts, a fraction of the subchannels is used by the macrocell layer, while another fraction is used by the femtocells as in WiMAX femtocells [29]. Spectrum allocation can be either static or dynamic, it can be 
static, depending on the geographic area, or can be made dynamic depending on the traffic load and user mobility. It can eliminate cross-layer interference leaving behind only co-layer interference. This approach is inefficient in terms of spectrum reuse, though it is optimal from a cross-layer interference standpoint [16].

Frequency reuse, as a simple way of spectrum sharing among cells, has been extensively studied to reduce the inter-cell interference for wireless systems. The traditional frequency reuse strategy was presented in [30] on cellular clustering. A larger cluster size leads to greater reduction of the inter-cell interference as well as the spectrum efficiency. An adaptive fractional frequency reuse (AFFR) scheme in [16], allocated spectrum bandwidth proportional to the traffic load of each cell in the multi-cell environment. A dynamic frequency reuse scheme was proposed in [31], in which each femtocell has been allocated equal spectral bandwidth to improve the cell-edge coverage quality. Graph-based resource allocation method for multi-cell environments was introduced in [32], which formulated the orthogonal resource allocation procedure as a NP hard vertex coloring problem of the adjacent cells. In [33], the impact of scheduling information exchange for clustered femtocells is investigated; in which macrocells operate in FDD (Frequency Division Duplex) mode and femtocells operate in the macro uplink band in TDD (Time Division Duplex) mode with the purpose of interference avoidance.

We will be using distributed, non-cooperative co-channel assignment for femtocell users in our work.

\subsubsection{Multiple Access Method}

Access control mechanisms have a direct effect on the interference of a network and plays an important role in mitigating cross-tier interference and handover attempts, that is why they have to be carefully chosen depending on the customer profile. Femtocells can be configured in three ways to allow or restrict their usage by certain users [29], [34]:

- Open access: where all the users can access all the femtocells of a given operator. 
- Closed access: where only the subscriber of the femtocell and a list of invited users can access a given femtocell.

- Hybrid access: where a limited amount of the femtocell resources are available to all users.

Wrong selection of an access method can result in network efficiency deterioration giving rise to severe interference among two-tier users. Therefore, an optimum choice of an access method is required to reduce both cross-tier and co-tier interferences. In [35], two types of access methods, closed and open access for OFDMA femtocells are presented and compared. While in [36], hybrid access method is presented and can be seen as a trade off between open and closed approaches, and reach a compromise between the impact on performance of subscribers and the level of access that is granted to non-subscribers. We will be using closed access method for the femtocell users to get connected to their home FAP.

\subsubsection{Interference Avoidance and Management}

Femtocell not only provides coverage at the customer premises, but also radiates toward neighboring houses as well as outdoors, introducing interference. Due to this and given that femtocells are deployed within the coverage area of existing macrocells, they can cause strong degradation of the macrocells performance. Furthermore, the deployment of new femtocells could also disturb the normal functioning of already existing femtocells. The opportunistic location of femtocells suggests that randomness must be taken into account when analyzing femtocell related issues.

Intra-system and inter-system interferences among femtocells and, between macrocells and femtocells respectively, is a challenging problem. Especially when femtocells are densely deployed and their positions are random rather being planned. Therefore, in order to reduce the appearance of "dead-zones" (coverage holes) within the macrocells and to successfully deploy a femtocell network, interference avoidance, randomization, or cancellation techniques 
must be applied.

The use of sectorized antennas at FAPs has been suggested in [37] as a means of reducing interference by decreasing the number of interferers. Similarly, a dynamic selection of predefined antenna patterns has been used in [38] to reduce the power leakage outdoors. These patterns are employed to adapt the femtocell coverage in different scenarios, thus avoiding cross-tier interference towards passing users. However, hardware-based approaches usually imply an increase in the femto BS cost [39]. On the other hand, strategies based on interference avoidance also represent efficient alternatives, e.g., power and subchannel management. Power control algorithms and radio resources management are tools often used in cellular systems to mitigate interference. If they are not applied, users located far from a base station will be jammed by users in much closer positions [17]. We use the orthogonal channel assignment among one FAP's users and also for all macrocell users, as an interference avoidance technique.

\subsection{Multicarrier Femtocell System}

In recent years, there have been tremendous efforts in research activities related to multicarrier transmission techniques, which has been proven to increase the application bit rates of wireless communications. The idea of multicarrier transmission is used for combating the hostility of wireless channels. It provides improved performance in bad channel conditions, high data rates and efficient bandwidth utilization. Avoidance of multipath propagation problem by using simple equalization at the receiver and computation efficiency, i.e., more efficient than single carrier transmission are the main advantages of multicarrier transmission [40]. In the following sections, we will be discussing various multicarrier techniques and their usefulness in femto/macro networks from the literature. 


\subsubsection{OFDMA Femtocells}

One of the main impairments of wireless channels is frequency selective fading. It is especially intense in multipath environments, where the behavior of the channel differs between different frequencies, and this is particularly true in indoor and urban environments. OFDMA radio access offers robustness against multipath fading and high spectral flexibility. Due to these benefits, OFDMA femtocells have proven to be highly versatile when dealing with various co-channel interferences in the deployment of two-tier femto/macro networks [11]. The available spectrum is divided into orthogonal subchannels, then grouped into subcarriers; multi-access is possible by allocating different users to different groups of orthogonal subchannels. Moreover, OFDMA femtocells can exploit channel variations in time domain. The intra-cell interference is avoided since users are assigned with unique frequency resource within a cell. Only the inter-cell interference should be taken into account in this case.

In order to increase the spectral efficiency of outer users in OFDMA femtocell system, fractional frequency reuse (FFR) has been proposed and extensively studied in [30], [41] and [42] for the local area scenarios in the LTE-Advanced system. Moreover, to minimize interference in femtocell deployments, use of sophisticated self-organization and selfoptimization techniques are needed. In [10] and [11], self-organizations and self optimization of OFDMA femtocells are discussed. The authors purposed the exchange of information between the femtocells and measurement reports sent by users. Effects of reduction in transmission power on the inter-cell interference in OFDMA femtocells were studied in [43] and [44].

\subsubsection{CDMA and MC-CDMA Femtocells}

As discussed earlier CDMA is a spread spectrum system, where the transmitted signal is spread over a wide frequency band, much wider than the bandwidth of the information being sent. It has been shown to be spectrally efficient through the flexible frequency reuse and multiple access technique. CDMA networks (without femtocells) employ fast power control 
to compensate for path-loss, shadowing and fading, and to provide uniform coverage. When femtocells are added, this interference-limited system will be greatly affected by the presence of femto/macro users and require efficient interference avoidance techniques such as timehopping or power control. "Dead-zones" are created due to inaccurate power control leading to non-uniform coverage [35]. Also "loud neighbour" problem is reported on the uplink, as a cell edge macrocell user transmitting at maximum power causes unacceptable interference to nearby femtocells. Consequently, cell edge femtocells experience significantly higher interference compared to interior femtocells. On the forward link, at the cell edge where femtocells are most needed, macrocell users are disrupted by nearby femtocell transmissions, since they suffer higher path-loss compared to cell interior users.

Several researchers are addressing these issues and working on possible feasible deployment of CDMA femtocells. Such as, macrocell and femtocell outage probabilities were derived, and load balancing for an open access CDMA network showing significant capacity improvement was discussed in [45]. Joint power and admission control solution for distributed interference management in two-tier CDMA-based networks was discussed in [46]. While the comparison between open and closed access CDMA femtocells was made in [35], it was concluded that open access CDMA femtocell is favorable by reducing the near-far problem experienced by the femtocell.

Another major issue regarding CDMA femtocells is the user mobility in terms of seamless handsoff between macrocells and femtocells. Soft handsoff (HO) is not possible, due to lack of common base station controller and also heavy reuse of PN codes, among femtocells causing ambiguity in choosing the handsoff target. The ambiguity in performing an active $\mathrm{HO}$ is solved by the uplink sensing at femtocells as has been proposed in [47].

MC-CDMA combines orthogonal frequency-division multiplexing (OFDM) and spread spectrum techniques and it inherits several competitive properties, e.g., robustness against the frequency selective fading channel, flexibility in system design, low detection complexity, lower peak-to-average power ratio and good probability of error performance in frequency 
selective fading channels. Due to above mentioned benefits, it is utilized in macro/femto network. Evaluation of system performance for various OFDM-CDMA systems operating over two-tier femtocells has been investigated in [12] and [13]. It was reported that, MCCDMA experiences performance degradation due to MAI.

\subsubsection{Two Dimentional (2D) Spreading}

Spreading the information symbols to be transmitted with the aid of orthogonal codes is the basis of supporting multiple access capabilities of multicarrier CDMA systems [48]. Instead of transmitting each complex symbol delivered by the modulator separately on a specific subchannel as in the OFDM modems, its influence is spread over several subcarriers with the aid of orthogonal multichip spreading codes.

Two dimensional spreading in two-tier femtocell networks has been investigated to some extent in the prior research. For example, impact of time-hopping on the uplink outage probability has been characterized for a macrocell/femtocell coexistence scenario in [45]. Capacity analysis of two neighboring femtocell networks (one of them being under-utilized, and the other being fully-utilized) with different spreading factors has been investigated in [49]. It has been shown that the spreading always improves the capacity of an underutilized femtocell, while it may or may not improve the capacity of a neighboring fully-utilized femtocell depending on the severity of the interference.

In our analysis, we utilize the two dimensional spreading for the femtocell users. Data streams from users are spread in time as well as in frequency, by modulating small and non-contiguous subcarrier groups. Spreading in time is done by using time domain $N_{t}$ and frequency domain $N_{f}$ chips. This two dimensional spreading does not only enhance efficient spectrum allocation but also avoids MAI and provides frequency diversity. For the first time, in our analysis the optimal spreading factor of an femto users is investigated from macro BER perspective. 


\subsection{Comparison of Multicarrier Femtocell Systems}

As discussed in section 3.2.2, CDMA networks are interference-limited and their performance has a fragile dependence on power control. Without accurate centralized power control, the near-far effect causes nearby users to overwhelm the received power of farther users, since they use the same band. With femtocells, such centralized power control is nearly impossible to accomplish because the received power levels cannot be simultaneously equalized at numerous points in space. For example, an uplink macrocell mobile user may transmit at a power level that effectively disables many nearby femtocells in that band. Therefore, adding even a small number of CDMA femtocells can have a profound impact [40].

While in OFDMA as discussed in section 3.2.1, large quantity of dynamically allocated time and frequency slots are present thus increasing the flexibility of resource allocation. This is beneficial as femtocells can be allocated orthogonal resources to nearby macrocells, the possibility for fine-tuned interference management exists, whereas it did not in CDMA. Potentially, a complex network-wide optimization and large amount of coordination among femto and macro users is necessary to avoid using those time and frequency slots used by the other network users. A popular compromise is fractional frequency reuse [31], whereby frequency or time resources can be semi-statically allocated to interior, edge, or small cell users, with power control on top to lower the throughput disparities experienced in each of these scenarios.

In Table 3.1, various multicarrier techniques are listed and their pros and cons are presented [40], [50]. It is obvious from the table that MC-CDMA can significantly outperform DS-CDMA with respect to BER performance and bandwidth efficiency in the synchronous downlink. It avoids ISI and intercarrier interference (ICI), allowing an efficient and simple user signal separation. It requires powerful receivers with low complexity due to the avoidance of ISI and ICI in the detection process [40]. Moreover, the spreading code length needs not to be necessarily equal to the number of subcarriers, which enables a flexible system design and can further reduce the complexity of the receiver. 


\begin{tabular}{|c|c|c|c|}
\hline Features & MC-CDMA & DS-CDMA & OFCDM \\
\hline Spreading & $\begin{array}{l}\text { Frequency } \\
\text { domain }\end{array}$ & Time domain & $\begin{array}{l}\text { Frequency } \\
\text { and time } \\
\text { both }\end{array}$ \\
\hline $\begin{array}{l}\text { BER re- } \\
\text { quirement } \\
\text { at given } \\
\text { Eb/No }\end{array}$ & Moderate & Higher & Lower \\
\hline $\begin{array}{l}\text { BW effi- } \\
\text { ciency }\end{array}$ & $\begin{array}{l}\text { Spreading in } \\
\text { frequency do } \\
\text { not require } \\
\text { extra BW }\end{array}$ & $\begin{array}{l}\text { Require extra } \\
\text { BW }\end{array}$ & $\begin{array}{lr}\text { Does } & \text { not } \\
\text { require } & \text { extra } \\
\text { BW } & \end{array}$ \\
\hline $\begin{array}{l}\text { MMSE } \\
\text { Equaliztion }\end{array}$ & Moderate & Moderate & Better \\
\hline $\begin{array}{l}\text { Receiver } \\
\text { design }\end{array}$ & Simple & Complex & Simple \\
\hline ICI and ISI & Avoid & Present & Avoid \\
\hline Interference & $\begin{array}{l}\text { Sensitive } \\
\text { to intracell } \\
\text { interference }\end{array}$ & Less sensitive & Less sensitive \\
\hline $\begin{array}{l}\text { Received } \\
\text { energy } \\
\text { utilization }\end{array}$ & $\begin{array}{l}\text { Employ all } \\
\text { the received } \\
\text { signal energy } \\
\text { scattered in } \\
\text { the frequency } \\
\text { domain. }\end{array}$ & $\begin{array}{l}\text { Difficiilt to } \\
\text { utilize full re- } \\
\text { ceived signal } \\
\text { energy scat- } \\
\text { tered in the } \\
\text { time domain }\end{array}$ & $\begin{array}{lr}\text { Can } & \text { fully } \\
\text { utilize } & \text { the } \\
\text { received } & \\
\text { energyan } & \end{array}$ \\
\hline Benefit & $\begin{array}{l}\text { Frequency di- } \\
\text { versity }\end{array}$ & No MAI & $\begin{array}{l}\text { Frequency } \\
\text { diversity and } \\
\text { robustness to } \\
\text { MPI and less } \\
\text { severe MAI }\end{array}$ \\
\hline Drawback & $\begin{array}{l}\text { MAI in fre- } \\
\text { quency selec- } \\
\text { tive channels }\end{array}$ & $\begin{array}{l}\text { Severe MPI } \\
\text { and MCI in } \\
\text { frequency } \\
\text { selective } \\
\text { channel }\end{array}$ & $\begin{array}{l}\text { MAI present } \\
\text { when or- } \\
\text { thogonality } \\
\text { among sub- } \\
\text { carrier is } \\
\text { lost }\end{array}$ \\
\hline
\end{tabular}

Table 3.1: Comparison of Various Multicarrier Techniques for Femtocells 
By comparing MC-CDMA and OFCDM, we note that the latter outperforms the former due to couple of reasons. The performance of MC-CDMA systems degrades with high channel loads due to the excessive MAI when transmitting through frequency channels [24]. This is not seen in OFCDM as it inherits all the advantages of OFDM, and also additional benefits by means of $2 \mathrm{D}$ spreading. Since OFCDM employs time and frequency domain spreading simultaneously, it experiences the favorable qualities of both MC-CDMA and MC-DS-CDMA systems. For example, frequency diversity gain can be achieved through frequency domain despreading due to the different fading experienced by subchannels in a broadband channel. Furthermore, with the introduction of time domain spreading, the system can provide flexible transmission rates and multiple access with low interference. OFCDM provides the best utilization of physical layer resources by accommodating a greater number of users. Another significant revelation is that an OFCDM symbol can be downgraded to either an CDMA symbol by using $S F_{\text {time }}=1$ and even to an OFDM symbol by using $S F_{\text {freq }}=1$ [50]. As a result of these benefits, OFCDM has been shown to provide more favorable BER performance than MC-CDMA and MC-DS-CDMA systems [8,28].

With the above mentioned benefits, OFCDM appears to be the best multicarrier technique. By inspecting the review from aforementioned research in the literature, it is noted that to analytically evaluate the system performance of an OFCDM with two-tier femtocell network, is sparse. It motivates us to further research a scenario of two layer OFCDM femtocells and OFDM macrocells users in this thesis.

In the next chapter, we will discuss in detail the system model for the OFDM and VSFOFCDM systems used in our research. It also includes the resource allocation strategy utilized in our analysis. 


\section{Chapter 4}

\section{Transceiver Model for OFDM Macro and VSF-OFCDM Femto Cells}

In this chapter, we outline in detail the system model used by the femto and macro UEs. First, a brief description of OFDM system model adopted by macro UEs is given. Following this, we provide the system model for the VSF-OFCDM system adopted by femto UEs. This includes description of the transmitter and receiver and channel model used. We also discuss the subcarrier grouping strategy that was earlier purposed in [26] is used to maximize the frequency diversity and minimize MAI in OFCDM system.

\subsection{OFDM System Model for Macro Users}

In this section, the system model for the OFDM system is presented. Figure 4.1 shows the block diagram of transmitter and receiver of an OFDM system. We have assumed that macro UEs are utilizing OFDM for their spectrum allocation and the system operates in the uplink, therefore the OFDM transmitter is located at the macro UE, and the receiver is at the MBS. 


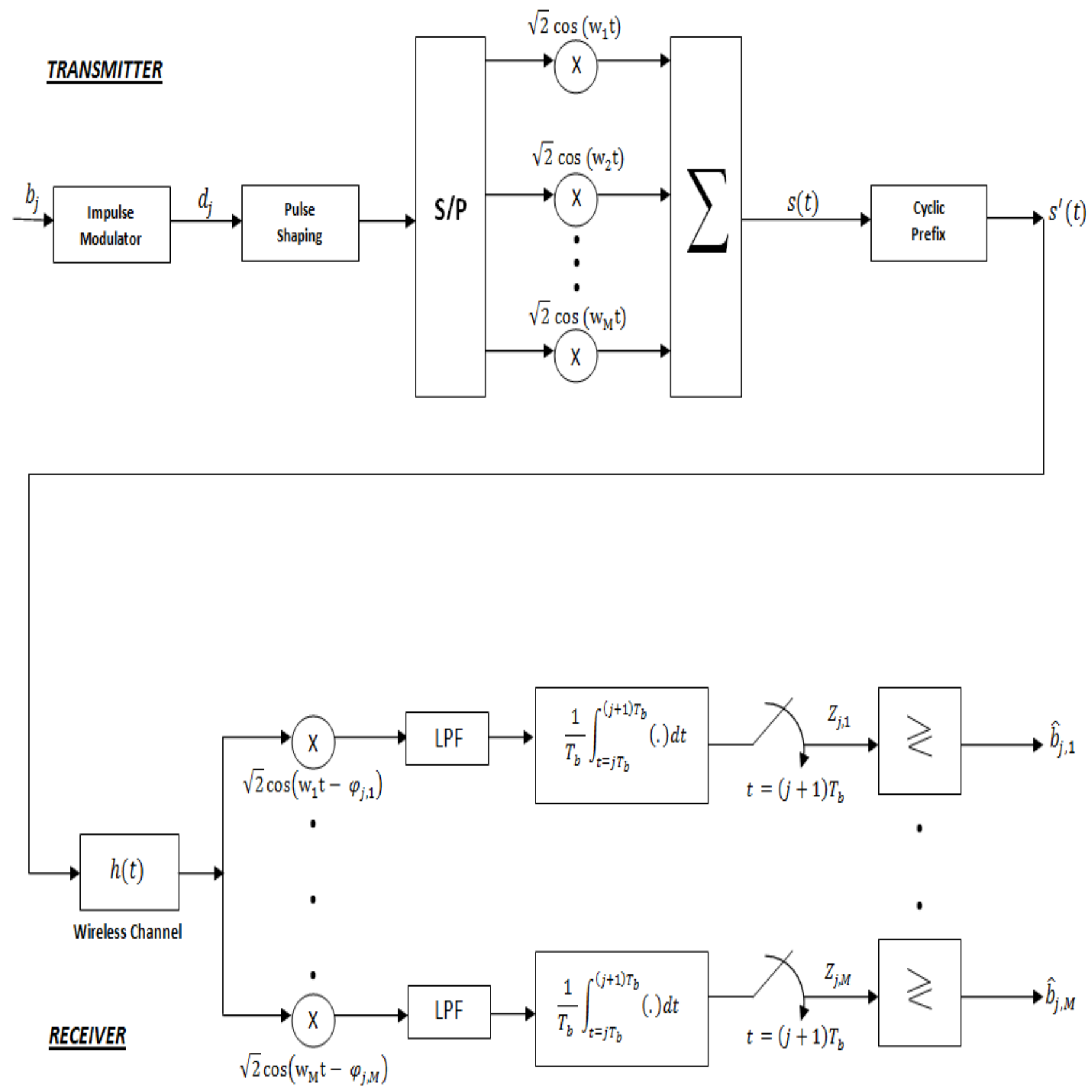

Figure 4.1: Transmitter/Receiver of OFDM System 


\subsubsection{Orthogonal Channel Assignment}

Since the macro users are using OFDM, orthogonal channels are assigned to them. The total number of subcarriers is distributed evenly among all the macro users. Users pick up the subcarrier from the total subcarriers assigned to them, on the basis of best channel gain criteria.

\subsubsection{OFDM Transmitter}

An OFDM signal is a sum of subcarriers that are individually modulated. Initially, incoming binary data stream from the user $l$ is impulse modulated producing sequence of complex modulated symbols having unique amplitude and phase. Then these complex modulated symbols are passed through the pulse shaping filter and also converted into parallel format. Afterwards each symbol is modulated by multiplying it, by a cosine function at the intended center frequency, to produce sinusoidal subcarriers at frequencies $\omega_{m}$, where $m \epsilon\{1,2, \ldots, M\}$ is the subcarrier index and $M$ is the total number of subcarriers. These orthogonal subcarriers are then summed to produce the OFDM signal for the $l^{\text {th }}$ user:

$$
\begin{aligned}
s^{(l)}(t) & =\sqrt{2 \varepsilon_{b}} \sum_{j} \sum_{m=1}^{M} d_{j, m}^{(l)} p\left(t-j T_{b}\right) \cos \left(\omega_{m} t\right) \\
& =\sqrt{2 \varepsilon_{b}} \sum_{j} \sum_{m=1}^{M}\left|d_{j, m}^{(l)}\right| p\left(t-j T_{b}\right) \cos \left(\omega_{m} t+\angle d_{j, m}\right),
\end{aligned}
$$

where $\varepsilon_{b}$ is the energy per bit, $T_{b}$ is the OFDM symbol duration, $d_{j, m}^{(l)}$ represents the $j^{\text {th }}$ complex symbol on the $m^{t h}$ subcarrier and $p\left(t-j T_{b}\right)$ is the symbol pulse shape. If BPSK modulation is utilized on the subcarriers than it simplifies the transmitted signal to:

$$
s^{(l)}(t)=\sqrt{2 \varepsilon_{b}} \sum_{j} \sum_{m=1}^{M} b_{j, m}^{(l)} p\left(t-j T_{b}\right) \cos \left(\omega_{m} t\right),
$$

where $b_{j, m}^{(l)}$ is the $j^{t h}$ bit on the $m^{t h}$ subcarrier. Afterwards a cyclic extension is added with $s(t)$ to avoid the intersymbol interference. Since the duration of the cyclic prefix is at least equal 
to the channel's memory length, all ISI occur during the cyclic prefix, leaving the OFDM symbol completely unaffected by ISI [19]. Also, the cyclic prefix aids in synchronization at the receiver because it can be utilized to identify the beginning of an OFDM symbol.

\subsubsection{Outdoor Channel Model}

After the OFDM modulation and the cyclic extension is added, $s^{\prime}(t)$ is the transmitted signal through the wireless channel. If $f_{c}$ is the carrier frequency (in $\mathrm{Hz}$ ) then the resultant transmitted signal is given as:

$$
s_{R F}^{(l)}(t)=s^{\prime}(t) \sqrt{2} \cos \left(2 \pi f_{c} t\right),
$$

We assume that the coherence bandwidth of the channel is equivalent to the subcarrier bandwidth $\left(1 / T_{b}=B_{c}\right)$. Based on this assumption, the channel model for the $m^{\text {th }}$ subcarrier during the $j^{\text {th }}$ transmitted bit is flat and the frequency domain response for an $l^{\text {th }}$ user is given as [21]:

$$
H_{j, m}^{(l)}=\alpha_{j, m}^{(l)} e^{i \varphi_{j, m}^{(l)}}
$$

where $\alpha_{j, m}^{(l)}$ is the fading gain, and $\varphi_{j, m}^{(l)}$ is the phase shift of the $m^{\text {th }}$ subcarrier during the $j^{\text {th }}$ transmitted bit. It is assumed that additive white gaussian noise (AWGN) having power spectral density (PSD) of $N_{o} / 2$ is present in the channel. We represent this noise signal as $\eta(t)$ and let the total impulse response over the $M$ subcarriers is denoted by $h(t)$. Thus the received signal is given as:

$$
r_{R F}^{(l)}(t)=s_{R F}(t) * h(t)+\eta(t)
$$

Then signal is down-converted and low pass filtered to remove the image signal (due to demodulation). Afterwards removal of cyclic prefix is done and the recovered signal is given as:

$$
r^{(l)}(t)=\sqrt{2 \varepsilon_{b}} \sum_{j} \sum_{m=1}^{M} \alpha_{j, m}^{(l)} b_{j, m}^{(l)} p\left(t-j T_{b}\right) \cos \left(\omega_{m} t+\varphi_{j, m}^{(l)}\right)+\eta(t) .
$$




\subsubsection{OFDM Receiver}

The received OFDM signal $r(t)$, is first converted into parallel format, and restored to the baseband by multiplying with cosine functions matched to the frequency $\left(\omega_{m}\right)$ and phase $\left(\varphi_{j, m}^{(l)}\right)$. The signal is then integrated over the bit period and sampled to generate the test statistic for the $j^{\text {th }}$ bit on the $m^{t h}$ subcarrier for the $l^{\text {th }}$ macro user.

$$
\begin{aligned}
Z_{j, m}^{(l)} & =\frac{1}{T_{b}} \int_{t=j T_{b}}^{(j+1) T_{b}} r(t) \sqrt{2} \cos \left(\omega_{m} t-\varphi_{j, m}^{(l)}\right) d t \\
& =\sqrt{\varepsilon_{b}} \alpha_{j, m}^{(l)} b_{j, m}^{(l)}+\eta_{j, m}
\end{aligned}
$$

where $\eta_{j, m}^{(l)}$ is the noise component during the $j^{t h}$ transmitted bit on the $m^{t h}$ subcarrier. An estimate of the received bit, $\hat{b}_{j, m}^{(l)}$, is based on the test statistic, $Z_{j, m}^{(l)}$, as follows:

$$
\hat{b}_{j, m}^{(l)}= \begin{cases}+1, & \text { if } Z_{j, m} \geq 0 \\ -1, & \text { if } Z_{j, m}<0\end{cases}
$$

From (4.7) and (4.8) the signal power at the output of the integrator is $\varepsilon_{b}\left(\alpha_{j, m}^{(l)}\right)^{2}$, and the noise power is $N_{o}$. Thus SNR $\gamma_{j, m}^{(l)}$, of an $l^{\text {th }}$ user for the $j^{\text {th }}$ bit (or symbol) on the $m^{\text {th }}$ subcarrier is given as:

$$
\gamma_{j, m}^{(l)}=\frac{\varepsilon_{b}\left(\alpha_{j, m}^{(l)}\right)^{2}}{N_{o}} .
$$

By substituting this value into the BER equation, the resulting probability of bit error during the $j^{\text {th }}$ signal element on the $m^{t h}$ subcarrier is given as [26]:

$$
P_{b_{j, m}}^{(l)}=Q\left(\alpha_{j, m}^{(l)} \sqrt{\frac{2 \varepsilon_{b}}{N_{o}}}\right) .
$$

\subsection{VSF-OFCDM System Model for Femto Users}

In this section, the system model for the VSF-OFCDM system is presented. Figures 4.3 and 4.4 show transmitter and receiver of an OFCDM system, respectively. We have assumed 
that femto users are utilizing VSF-OFCDM for their spectrum allocation and the system operates in the uplink, so the OFCDM transmitter is located at femto UE, and the receiver is at the FAP.

\subsubsection{Channel Grouping and Assignment}

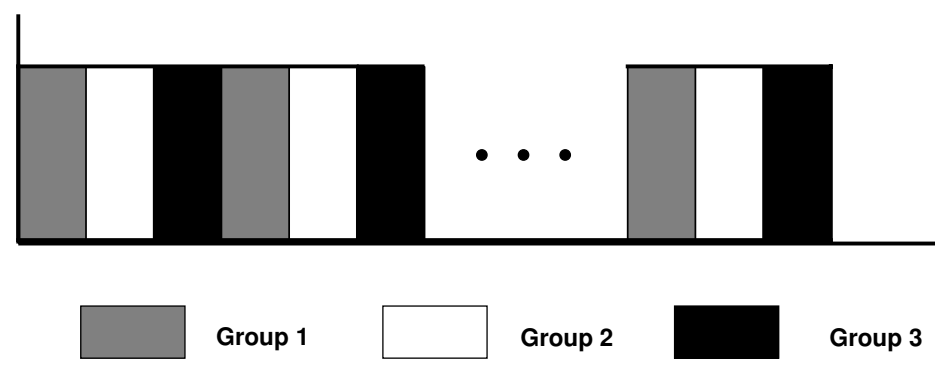

Figure 4.2: Subcarrier grouping with 3 subcarrier groups.

In [26], the authors proposed the use of subcarrier grouping to improve the performance of the OFCDM system. Using this strategy in our research for the spectrum allocation of femto users, the total number of subcarriers is divided into several small, non-contiguous groups. In particular, the total spectrum is divided into $G_{y}$ groups; each group $y$ has $M$ non-contiguous subcarriers that are equally spaced throughout the spectrum, the spectral separation of the subcarriers in the same group is identical to the total number of groups. The user groups, denoted $G_{y}, y \epsilon\{1,2, \ldots, Y\}$, contain the following subcarriers:

$$
\begin{gathered}
G_{1}=\left\{m_{1}, m_{1+\mu}, m_{1+2 \mu}, \ldots, m_{1+M_{1} \mu}\right\}, \\
G_{2}=\left\{m_{2}, m_{2+\mu}, m_{2+2 \mu}, \ldots, m_{2+M_{2} \mu}\right\}, \\
\vdots \\
G_{Y}=\left\{m_{Y}, m_{Y+\mu}, m_{Y+2 \mu}, \ldots, m_{Y+M_{Y} \mu}\right\},
\end{gathered}
$$

where there are $M_{y}$ subcarriers in group $y, Y$ is the total number of groups, and $\mu$ is the spectral separation between subcarriers. Following the subcarrier grouping strategy in [21] and [26], three subcarrier groups are formed as shown in Figure 4.2. 
Since the subcarriers in each group are separated equally throughout the bandwidth, the frequency spacing is likely to be larger than the coherence bandwidth of the channel. This ensures that the fading on all subcarriers in the same group is uncorrelated, allowing the system to achieve the maximum benefit from frequency diversity. Also, since the number of subcarriers in each group is small, the amount of MAI caused by frequency domain spreading is minimized [26].

\subsubsection{VSF-OFCDM Transmitter}

An OFCDM system with $K$ simultaneous users is considered. Each data symbol is spread in time domain with $N$ chips, where $N$ is equal to $N_{t}$ and in frequency domain with $N_{f}$ chips which are equal to $M$ subcarriers in each group. At the transmitter, bit stream for user $k$, during the $j^{\text {th }}$ signal element is encoded and modulated and then processed by symbol interleaver. A symbol interleaver is employed to randomize the deep faded symbols due to frequency selective fading. Therefore, the frequency diversity can be achieved in frequency domain to improve the performance [21]. Interleaved data symbols are then S/P converted. After $\mathrm{S} / \mathrm{P}$ conversion, 2D spreading is performed, where each data symbol is first spread into chips in time domain with the spreading code $C^{T(k)}$ and then the time domain spread signal is duplicated into $M$ copies and each copy is multiplied by a chip of the frequency domain spreading code $C^{F(k)}$. The spread signal from different signals is added and up-converted into selected subcarriers and finally a complete OFCDM signal is obtained as shown in (4.12). Therefore, assuming BPSK modulation, the transmitted signal for the $k^{t h}$ user can be written as:

$$
S_{(t)}^{(k)}=\sqrt{\left(2 \varepsilon_{c}\right)} \sum_{j} b_{j}{ }^{(k)} \sum_{m=1}^{M} v_{j, m}^{(k)} C_{j, m}^{F(k)} \cos \left(\omega_{m} t\right) \sum_{n=1}^{N} C_{j, m, n}^{T(k)} p\left(t-(j N+n) T_{c}\right)
$$

The binary data stream for user $k, b_{j}(k)= \pm 1$ and $\mathbf{d}_{j}^{(k)}$ is the sequence of complex modulated symbols after BPSK modulation. Since BPSK modulation is utilized, the sequence $\mathbf{d}_{j}^{(k)}=$ 


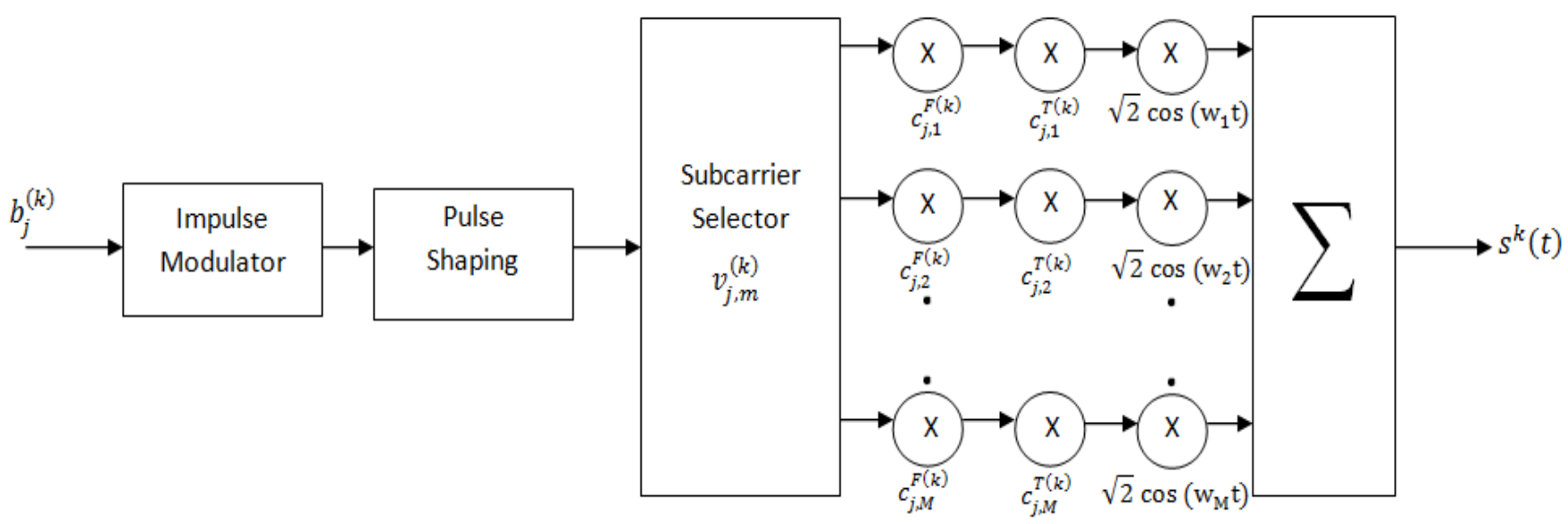

Figure 4.3: Transmitter of OFCDM System

$\pm 1, j \in \aleph$. Also, $\omega_{m}$ is the frequency of the $m^{t h}$ subcarrier, $C_{j, m, n}^{T(k)}$ is the $n^{t h}$ chip in the time domain spreading code on the $m^{t h}$ subcarrier and $C_{j, m}^{F(k)}$ is the $m^{\text {th }}$ chip in the frequency domain. $\varepsilon_{c}$ is the chip energy, and the parameter $v_{j, m}^{(k)}$ is defined as:

$$
v_{j, m}^{(k)}= \begin{cases}1, & \text { if } m \epsilon G_{y} \\ 0, & \text { otherwise }\end{cases}
$$

More specifically, $v_{j, m}^{(k)}=1$ if the $m^{t h}$ subcarrier is assigned to femto user $k$. If the subcarrier is not allocated to user $k$ during the $j^{t h}$ signal element, $v_{j, m}^{(k)}=0$.

\subsubsection{Indoor Channel Model}

In this analysis, the channel is assumed to be slowly varying with respect to the OFCDM symbol duration, $T_{b}$. We also assume that the delay spread is smaller than the time domain $P N$ chip time, $T_{c}$. Therefore, each subcarrier experiences independent, frequency nonselective fading and the channel is modeled as Rayleigh fading channel. The impulse response of the channel for the $k^{\text {th }}$ user is given as:

$$
H_{(j, m)^{(k)}}=\alpha_{j, m}^{(k)} e^{i \phi(j, m)^{(k)}}
$$


where $\alpha_{j, m}^{(k)}$ is the Rayleigh fading gain for the $k^{\text {th }}$ user, on the $m^{\text {th }}$ subcarrier during the $j^{\text {th }}$ transmitted bit. This value is independent for all $K$ users and $M$ subcarriers. The phase, $\phi_{j, m}^{(k)}$, is a uniformly distributed random variable over $[0,2 \pi)$, which is independent for each bit, subcarrier and user.

\subsubsection{VSF-OFCDM Receiver}

Since we are considering the uplink, the transmitter is located at femto mobile terminal. Consequently, the signal received at the femto base station consists of a summation of signals from $K$ femto users transmitting simultaneously. Figure 4.4 shows the block diagram of an VSF-OFCDM receiver. The total received signal is written as:

$r(t)=\sqrt{\left(2 \varepsilon_{c}\right)} \sum_{k=1}^{K} \Sigma_{j} b_{j}^{(k)} \sum_{m=1}^{M} v_{j, m}^{(k)} \alpha_{j, m}^{(k)} C_{j, m}^{F(k)} \cos \left(\omega_{m}(t)+\psi_{j, m}^{(k)}\right) \sum_{n=1}^{N} C_{j, m, n}^{T(k)} p\left(t-(j N+n) T_{c}\right)+\eta(t)$,

where $\eta(t)$ is the AWGN noise signal with a double-sided power spectral density (PSD) of $N_{o} / 2 . K_{y}$ is the total number of users in a group $y, b_{j}^{(k)}$ is the BPSK signal for the $k^{t h}$ user during the $j^{\text {th }}$ transmitted bit. In order to recover the data for user $k$, convert the received signal from $\mathrm{P} / \mathrm{S}$ and multiply the subcarrier allocation coefficients for user $k, v_{j, m}^{(k)}$. The signal on the $m^{\text {th }}$ subcarrier is first correlated with the synchronized time domain spreading sequence $C_{j, m, n}^{T(k)}$ for user $k$. The output of each time domain correlator is then multiplied by its frequency domain spreading code $C_{j, m, n}^{F(k)}$ to remove the frequency domain spreading. The despread signal is then multiplied by the fading gain, $\gamma_{j, m}^{(k)}$ according to the maximal ratio combining (MRC) algorithm [26]. Finally, the subcarriers are summed, integrated over the bit period, and sampled to yield the decision variable for user $k$.

$Z_{j}^{(k)}=\frac{1}{T_{c}} \int_{t=j T_{b}}^{(j+1) T_{b}} \sum_{m=1}^{M} v_{j, m}^{(k)} \alpha_{j, m}^{(k)} c_{j, m}^{F(k)} \sum_{n=1}^{N} c_{j, m, n}^{T(k)} p\left(t-(j N+n) T_{c}\right) \operatorname{LPF}\left\{r(t) \sqrt{2} \cos \left(\omega_{m} t-\varphi_{j, m}^{(k)}\right)\right\} d t$ 


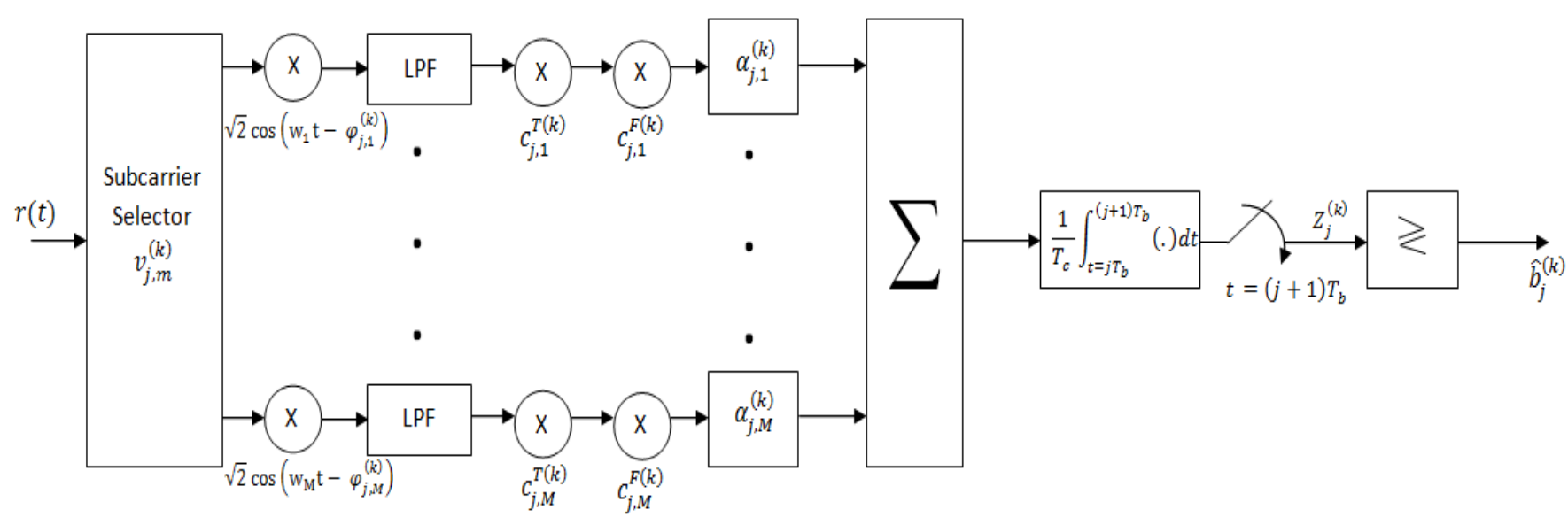

Figure 4.4: Receiver of OFCDM System

where LPF is the low pass filter to remove the image frequency caused by demodulation. The decision variable is comprised of desired signal of the $k^{\text {th }}$ user, interference signal and noise. We can express it in terms of the desired signal, $D_{j}^{(k)}$, interference term, $I_{j}^{(k)}$, and the noise term, $\eta_{j}^{(k)}$ as follows:

$$
Z_{j}^{(k)}=D_{j}^{(k)}+I_{j}^{(k)}+\eta_{j}^{(k)}
$$

The required desired signal at the output of the correlator is given as:

$D_{j}^{(k)}=\frac{1}{T_{c}} \int_{t=j T_{b}}^{(j+1) T_{b}} \sqrt{\varepsilon_{c}} b_{j}^{(k)} \sum_{m=1}^{M} v_{j, m}^{(k)}\left(\alpha_{j, m}^{(k)}\right)^{2}\left\{C_{j, m}^{F(k)} C_{j, m}^{F(k)}\right\} \sum_{n=1}^{N}\left\{C_{j, m, n}^{T(k)} C_{j, m, n}^{T(k)}\right\} p\left(t-(j N+n) T_{c}\right) d t$

The quantity $p\left(t-(j N+n) T_{c}\right)$ has a constant amplitude of unity over $T_{c},\left\{c_{j, m}^{F(k)} c_{j, m}^{F(k)}\right\}=1$ and $\left\{c_{j, m, n}^{T(k)} c_{j, m, n}^{T(k)}\right\}=1$, thus the decision variable is simplified to:

$$
D_{j}^{(k)}=N \sqrt{\varepsilon_{c}} b_{j}^{(k)} \sum_{m \in G_{y}}\left(\alpha_{j, m}^{(k)}\right)^{2}
$$

where $m \epsilon G_{y}$ represents the set of subcarriers that are in group $y$.

Due to the loss of orthogonality between the spreading sequences of different users we have an interference power. $I_{j}^{(k)}$ is calculated by considering the corelation between the $k^{t h}$ user 
and other users using the same group of subcarriers.

$$
\begin{aligned}
I_{j}^{(k)}= & \sqrt{\varepsilon_{c}} \sum_{k \epsilon G_{y}} b_{j}^{(k)} \sum_{m \epsilon G_{y}} \alpha_{j, m}^{\left(k_{y}\right)} \alpha_{j, m}^{(k)} C_{j, m}^{F(k)} C_{j, m}^{F\left(k_{y}\right)}\left[\cos \left(\varphi_{j, m}^{\left(k_{y}\right)}-\varphi_{j, m}^{(k)}\right)\right] \\
& \cdot \frac{1}{T_{c}} \int_{t=j T_{b}}^{(j+1) T_{b}} \sum_{n=1}^{N} C_{j, m, n}^{T(k)} C_{j, m, n}^{T\left(k_{y}\right)} p\left(t-(j N+n) T_{c}\right) d t
\end{aligned}
$$

where $k_{y} \epsilon G_{y}$ is the set of femto users that utilize group $y$ simultaneously. Similarly, noise power at the output of the correlator is given as:

$$
\begin{aligned}
\eta_{j}^{(k)}= & \sum_{m=1}^{M} \alpha_{j, m}^{(k)} C_{j, m}^{F(k)} \frac{1}{T_{c}} \int_{t=j T_{b}}^{(j+1) T_{b}} \sum_{n=1}^{N} C_{j, m, n}^{T(k)} p\left(t-(j N+n) T_{c}\right) \\
& \operatorname{LPF}\left\{n(t) \sqrt{2}\left[\cos \left(\omega_{m} t-\phi_{j, m}^{(k)}\right)\right] .\right.
\end{aligned}
$$

In the next chapter, we present simulation scenario for the hybrid macro/femto network and BER analysis of uplink interference among both types of users. Afterwards, SINR and BER expressions for femto and macro users are derived. 


\section{Chapter 5}

\section{BER Performance Analysis}

We analyze the BER performance of co-channel deployment of a two tier femto/macro network, under different spreading factors, channel loads and wall penetration losses. Also detail analysis of uplink interference is done and expressions for SINR and BER of femto and macro users are derived. These expressions will be used in Monte Carlo analysis in the next chapter.

\subsection{Hybrid Network Scenario}

A hybrid femto/macro network scenario ${ }^{1}$ under investigation is shown in Figure 5.1. The letter $M$ in the middle of a big circle is the MBS and squares on the circle are macro UEs. The letter $F$ in the middle of small circles are FAPs, while four circles inside are closed access femto UEs for the respective femto cell. The squares around femto circles shows the walls between the FAPs. This can be considered as a typical femto/macro hybrid configuration in an urban area, where several FAPs are deployed in the coverage area of a MBS. We have considered home FAPs, which can support three to five simultaneous users [2]. There is a very low probability that all the subscribers are simultaneously using the femtocell, which is why home femtocells supporting more than four simultaneous users is not realistic. Also,

\footnotetext{
${ }^{1}$ For simplicity, we assumed this simple network model in our analysis.
} 
we have considered closed access FAPs because residential users prefer femtocell in a private access mode that is only utilized by them.

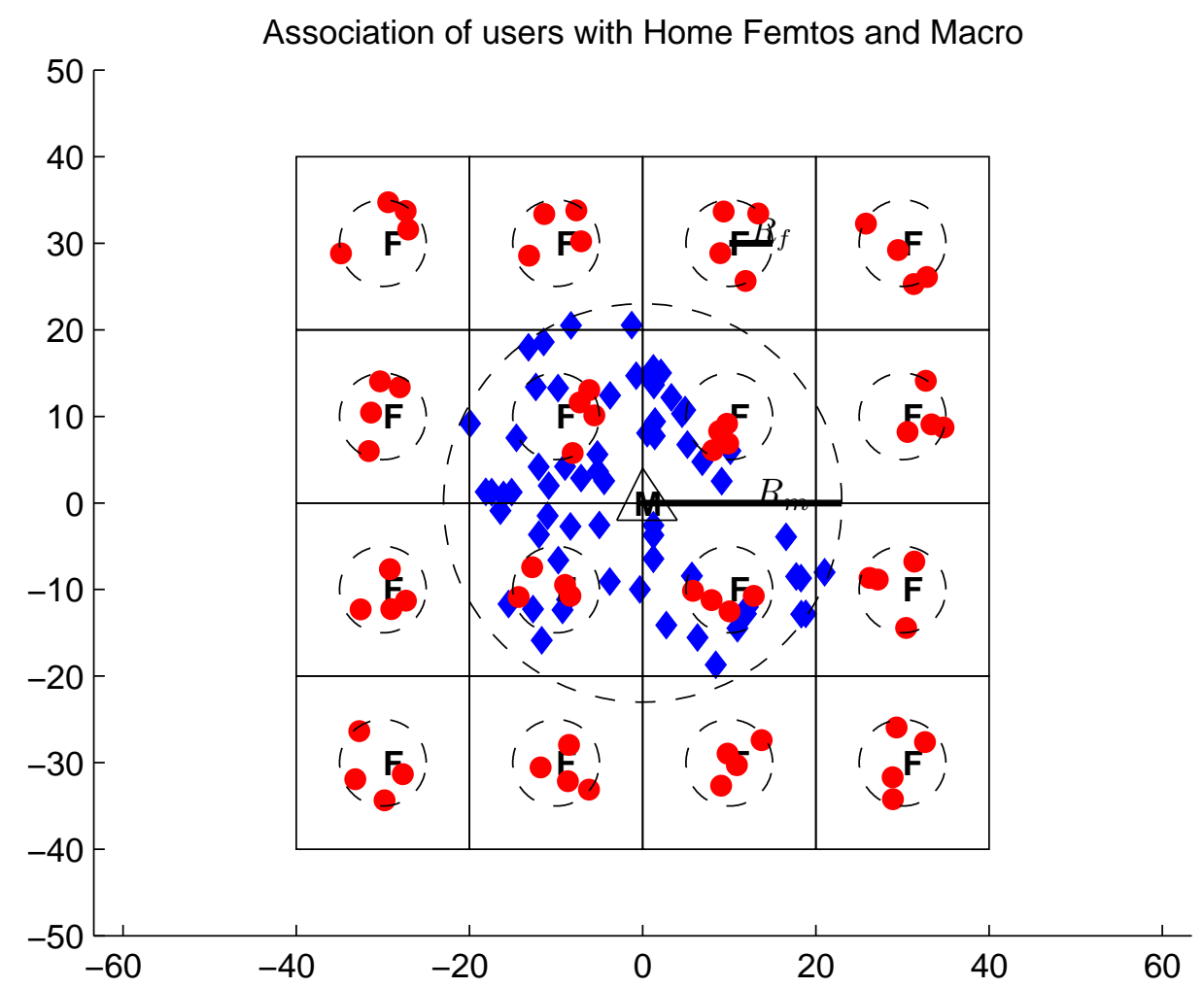

Figure 5.1: Femto/Macro Network Scenario

\section{Macrocell}

A single macrocell is considered with the base station (BS) located in the middle of the macrocell area and 64 macro users are uniformly distributed within its coverage area ${ }^{2}$. We also assume that the coverage area of MBS is a fixed circular area with radius $R_{m}$.

\footnotetext{
${ }^{2}$ Note that for simplicity of analysis, we assume this simple model, however, this analysis, approach and conclusion can be straightforwardly extended to any user configuration.
} 


\section{Femtocells}

There are 16 FAPs uniformly deployed over the entire area of a macrocell. Each FAP can serve up to four users making 64 femto users in total in the network. We assume that the value of femtocell coverage radius $R_{f}$ is fixed, and also $R_{f} \ll R_{m}$ as shown in Figure 5.2.

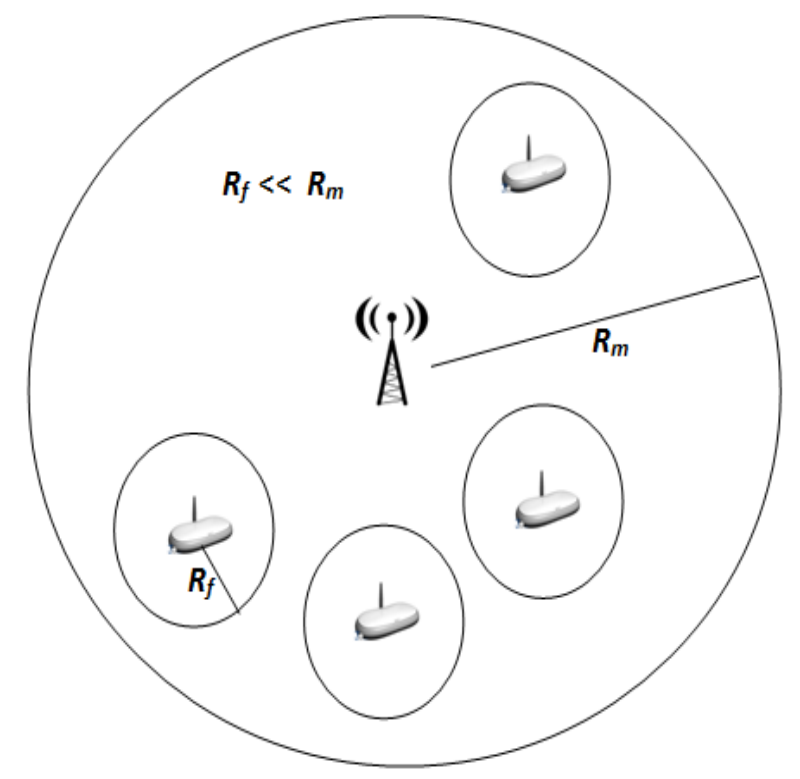

Figure 5.2: Coverage area of Macro and Femto Cells, $R_{f} \ll R_{m}$

\subsection{System Parameters}

Parameters for this two-tier system are the one typically used for $4 \mathrm{G}$ wireless system. The carrier frequency of the system used is $5 \mathrm{GHz}$ and uplink channel bandwidth is $20 \mathrm{MHz}$ that is sufficient to support the high rate applications. In total 64, 128 and 256 subcarriers are used in the allocated bandwidth and the resulting subcarrier bandwidth is $1 / T c=312.5 \mathrm{kHz}$, $156.25 \mathrm{kHz}, 78.1 \mathrm{kHz}$ which correspond to the chip duration of $3.2 \mu \mathrm{s}, 6.4 \mu \mathrm{s}$ and $12.8 \mu \mathrm{s}$ respectively. 


\section{Macrocell}

Macrocell is using OFDM for orthogonal channel assignment for its users as OFDM is the dominant cellular platform for the foreseeable future. Subcarriers are allocated using best gain criteria thus increasing the SINR efficiency of macro users.

\section{Femtocells}

Femtocells are using VSF-OFCDM for centralized channel assignment for their users. OFCDM has already proved itself to be the efficient multicarrier technique used in broadband channel [51]. Subcarriers are arranged into non-contiguous groups as mentioned in section 4.2.1. These are allocated to the users on the basis of best available channel gain criteria. Total spreading factor used is 32 and it is constant; while the time and frequency spreading factors are variable. The effect of different time and frequency spreading factors for different channel loads is analyzed. These different settings are used to provide different levels of frequency diversity or to minimize MAI in the event of high channel utilization.

\subsection{Channel Models}

In our analysis, we assume each subcarrier experiences frequency non-selective fading and that fading is uncorrelated between adjacent subcarriers. Consequently, evaluations are performed with an RMS delay spread of $6.4 \mu$ s which is common for densly populated urban area [26]. In this case, delay spread is identical to the chip duration, $T_{c}$, indicating that there is no ISI produced by the channel. This allows us to utilize independent, identically distributed Rayleigh variables to represent the gain of each subcarrier. Uplink channel from UE to the FAP/BS is composed of a possible deterministic wall-penetration loss component, a deterministic distance-dependent path-loss component (with a fixed path-loss exponent), and a random distance-independent component, which includes Rayleigh fading only. 


\section{Outdoor Model for Macrocell}

We assume that macro uplink transmission power $\left(P_{t}^{M}\right)$ is fixed. Therefore, MBS receiving power from one of its users is denoted by $P_{r}{ }^{M}=P_{t}{ }^{M} G^{M}$, where $G^{M}$ is the channel gain and is given as, $G^{M}=H_{l m} d_{l m}{ }^{-\alpha_{M}}$; path loss is denoted by $d_{l m}{ }^{-\alpha_{M}}$ and $H_{l m}$ is the channel gain between $l$ MUE and MBS. Also, MBS receives signal power $\left(P_{t}^{F_{k}}\right)$ from $k^{\text {th }} \mathrm{FUE}$, $P_{r}{ }^{M}(f)=P_{t}{ }^{F_{k}} G_{f}^{M}$, where $G_{f}^{M}$ is the channel gain and is given as, $G_{f}^{M}=H_{k m} d_{k m}{ }^{-\alpha_{F M}}$, where path loss is denoted by $d_{k m}{ }^{-\alpha_{F M}}$ and $H_{k m}$ is the channel gain between the $k$ FUE and MBS. We assume that wall penetration loss effect is for femto users only and both of them are using single input single output, omni-directional antennas with unity gain for transmission and reception.

\section{Indoor Model for Femtocells}

We assume that femto transmission power $\left(P_{t}{ }^{F}\right)$ is fixed. Therefore, femtocell $i$ receiving power from one of its users is denoted by $P_{r}{ }^{F_{i}}=P_{t}^{F} G^{F}$, where $G^{F}$ is the channel gain from user $k$ to FAP and given as, $G^{F}=H_{k f} d_{k f}{ }^{-\alpha_{F}} W_{f}$; path loss is denoted by $d_{k f}{ }^{-\alpha_{F}}$ (indoor), $H_{k f}$ is the Rayleigh random variable and $W_{f}$ is the wall penetration loss. In indoor environment, all users are relatively static or move slowly, therefore, we do not consider shadow fading or fast fading in our analysis. Therefore, FAP $i$ is receiving signal power $\left(P_{t}{ }^{M_{l}}\right)$ from $l^{\text {th }}$ MUE and it is denoted as $P_{r}{ }^{F_{i}}{ }_{(m)}=P_{t}{ }^{M_{l}} G_{m}^{F}$, where $G_{m}^{F}$ is the channel gain and is given as, $G_{m}^{F}=H_{l f} d_{l f}^{-\alpha_{M F}} W_{f}$; path loss is denoted by $d_{l f}{ }^{-\alpha_{M F}}, H_{l f}$ is the channel gain between the $l$ MUE and FAP.

\subsection{Uplink Interference Analysis}

In our work, we assume decentralized interference control and in this section we will analyze uplink interference of femtocell and macrocell UEs. 


\section{Macrocell Users}

Orthogonal subcarrier assignment is done for the macro users so that there is no MAI among them. Macrocell users are experiencing uplink interference only from the femtocell users using the same set of subcarriers. For simplicity, co-channel interference from neighboring macrocells transmissions is ignored with a single macrocell in our system model. Uplink interference of macrocell users is denoted as $I_{\text {macro }}=I_{F . M}+I_{M . M}$. Let $I_{F . M}$ be the interference from all femtocell users to MBS and $I_{M . M}$ be the interference from other macrocell users. Due to the fact that there is only one MBS in this cell and orthogonal channels are assigned to macro users, there is no intra-macro uplink interference, i.e $I_{M . M}=0$, therefore, $I_{\text {macro }}=$ $I_{F . M}$

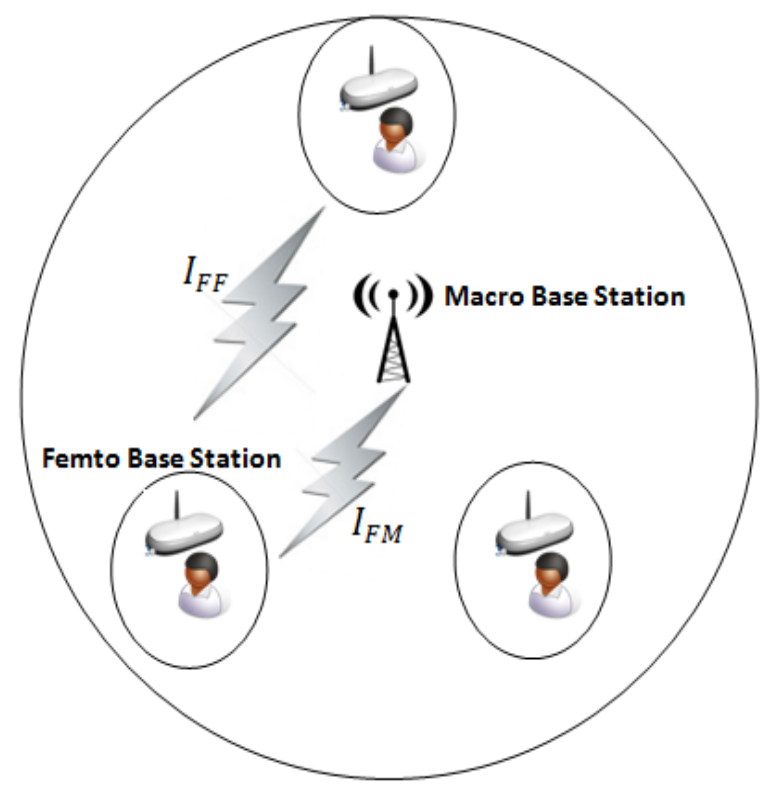

Figure 5.3: Interferences among Femto and Macro Users.

\section{Femtocells Users}

Closed access method is employed by the femto users for the channel access. All the femto users within a femtocell are assigned orthogonal subcarrier groups, thus neglecting the intracell interference within the femto cell. Only inter-cell interference among femtocell users and 
interference from neighboring macro users is affecting the femto SINR. Hence, in terms of femtocell $i$, uplink interference is only from users of the rest of the femtocells and the macro users adopting the same set of subcarriers. Uplink interference of femtocells is denoted as $I_{\text {femto }}=I_{M . F}+I_{F . F}$. Let $I_{M . F}$ be the interference at a FAP from macro users and $I_{F . F}$ be the interference from other femto users.

\subsection{BER Calculation}

In order to determine the BER performance of macro and femto users, the average SINR is calculated for each transmitted bit. The SINR is defined as the signal power divided by the interference and noise power. We derive the expression for SINR of femto as well macro users separately.

\subsubsection{SINR of Femto Users}

SINR of femto user during the $j^{\text {th }}$ signal element is defined as:

$$
\gamma_{j}^{F}=\frac{P_{j}^{F}}{I_{j}^{F}+N_{j}^{F}},
$$

where,

$N_{j}^{F}=$ noise power present at the femto (F) user during $j^{\text {th }}$ signal element,

$P_{j}^{F}=$ power of the desired signal received by femto $(\mathrm{F})$ user during $j^{\text {th }}$ signal element, $I_{j}^{F}=$ total interference power received by the femto user during the $j^{\text {th }}$ signal element $=$ $I_{j}^{F . F}+I_{j}^{F . M}$

$I_{j}^{F . F}=$ interference power received by femto $(\mathrm{F})$ user from other femtocell $(\mathrm{F})$ users sharing the same subcarrier group $y$,

$I_{j}^{F . M}=$ interference power received by femto $(\mathrm{F})$ user from macro $(\mathrm{M})$ users sharing the subcarrier present in the group $y$.

$P_{j}^{F}$ is obtained by taking the variance of the desired signal $D_{j}^{(k)}$, defined by (4.16). Since 
the desired signal has the mean of $0 \mathrm{~dB}$ that is normalized to 1 , the resulting signal power is given as [26]:

$$
P_{j}^{F}=\operatorname{Var}\left[D_{j}^{(k)}\right]=N^{2} \varepsilon_{c}\left[\sum_{m \in G_{y}}\left(\alpha_{j, m}^{(k)}\right)^{2}\right]^{2}
$$

Interference power $I_{j}^{F . F}$ from other femto users can be approximated as a Gaussian random variable as explained in [20], [52] for an OFCDM system and by using (4.18), it is given as,

$$
I_{j}^{F . F}=N \varepsilon_{c}\left(K_{y}-1\right) E\left[\left(\alpha_{y}\right)^{2}\right] \sum_{m \in G_{y}}\left(\alpha_{j, m}^{(k)}\right)^{2}
$$

where $K_{y}$ is the number of users occupying group $y$ simultaneously, and $E\left[\left(\alpha_{y}\right)^{2}\right]$ is the average fading gain for the $K_{y}$ users in group $y$.

Interference power $I_{j}^{F . M}$ from $L$ macro users utilizing OFDM for their spectrum sharing is obtained from (4.7) and (4.8). The interference signal power is given as $\varepsilon_{b}\left(\alpha_{j, m}^{(l)}\right)^{2}$. Finally, the noise power is calculated by finding the variance of the noise signal, $\eta_{j}^{(k)}$ from (4.19). The resulting noise power is given as:

$$
N_{j}^{F}=\operatorname{Var}\left[\eta_{j}^{(k)}\right]=N N_{o} \sum_{m \in G_{y}}\left(\alpha_{j, m}^{(k)}\right)^{2}
$$

where $N$ is the spreading in time domain. The received signal and interference power are affected by uplink channel gain, comprised of a possible deterministic wall-penetration loss component $W_{f}$, a deterministic distance-dependent path-loss component (with a fixed path-loss exponent) $d_{k f}^{-\alpha_{F}}$, and a random distance-independent component, which includes Rayleigh fading only. Including these parameters as described in section 5.3, SINR for femto user $k$ on group $y$ for $j^{\text {th }}$ bit is given as:

$$
\gamma_{j, G_{y}}^{(k)}=\frac{d_{k f}{ }^{-\alpha_{F}} W_{f} N^{2} \varepsilon_{c}\left[\sum_{m \epsilon G_{y}}\left(\alpha_{j, m}^{(k)}\right)^{2}\right]^{2}}{I_{j}^{F . F}+I_{j}^{F . M}+N_{j}^{F}}
$$


where,

$$
\begin{gathered}
I_{j}^{F . F}=\sum_{i=1}^{K_{y}} d_{i f}^{-\alpha_{F F}} W_{f} N \varepsilon_{c}\left(K_{y}-1\right) E\left[\left(\alpha_{y}\right)^{2}\right] \sum_{m \in G_{y}}\left(\alpha_{j, m}^{(k)}\right)^{2} \\
N_{j}^{F}=N N_{o} \sum_{m \in G_{y}}\left(\alpha_{j, m}^{(k)}\right)^{2} \\
I_{j}^{F . M}=\sum_{z=1}^{L} d_{z f}^{-\alpha_{M F}} \varepsilon_{b} L \Sigma_{m \in G_{y}}\left(\alpha_{j, m}^{(\ell)}\right)^{2}
\end{gathered}
$$

Also, $\alpha_{F F}$ is the path loss exponent between two interfering femto users and $\alpha_{M F}$ is the pathloss exponent between the interfering macro users and femto user. Dividing by $N \Sigma_{m \epsilon G_{y}}\left(\alpha_{j, m}^{(k)}\right)^{2}$ we get,

$$
\gamma_{j, G_{y}}^{(k)}=\frac{\frac{d_{k f}^{-\alpha} F W_{f} N^{2} \varepsilon_{c}\left[\sum_{m \epsilon G_{y}}\left(\alpha_{j, m}^{(k)}\right)^{2}\right]^{2}}{N \Sigma_{m \epsilon G_{y}}\left(\alpha_{j, m}^{(k)}\right)^{2}}}{\frac{I_{j}^{F \cdot F}}{N \Sigma_{m \epsilon G_{y}}\left(\alpha_{j, m}^{(k)}\right)^{2}}+\frac{I_{j}^{F \cdot M}}{N \Sigma_{m \epsilon G y}\left(\alpha_{j, m}^{(k)}\right)^{2}}+\frac{N_{j}^{F}}{N \Sigma_{m \epsilon G_{y}}\left(\alpha_{j, m}^{(k)}\right)^{2}}},
$$

By simplifying we get,

$$
\gamma_{j, G_{y}}^{(k)}=\frac{d_{k f}^{-\alpha_{F}} W_{f} N \varepsilon_{c} \Sigma_{m_{\epsilon} G_{y}}\left(\alpha_{j, m}^{(k)}\right)^{2}}{\sum_{i=1}^{K_{y}} d_{i f}^{-\alpha_{F F}} W_{f} \varepsilon_{c}\left(K_{y}-1\right) E\left[\left(\alpha_{y}\right)^{2}\right]+N_{o}+\left(\frac{\sum_{z=1}^{L} d_{z f}^{-\alpha_{M F} \varepsilon_{b} L \Sigma_{m \epsilon G_{y}}\left(\alpha_{j, m}^{(\ell)}\right)^{2}}}{N \Sigma_{m \in G_{y}}\left(\alpha_{j, m}^{(k)}\right)^{2}}\right)}
$$

where $m \epsilon G_{y}$ represents the set of subcarriers in group $y . L \epsilon G_{y, m}$ is the set of macro users utilizing $m^{\text {th }}$ subcarrier present in group $y$. As BPSK modulation is used by femto users, the probability of bit error can be determined for the $j^{\text {th }}$ bit as follows:

$$
P_{b j}^{(k)}=Q\left(\sqrt{2 \gamma_{j, G_{y}}^{(k)}}\right)
$$

It is evident from the above expression that the SINR is dependent on the fading gains for user $k$, the interference power on group $y$ and the wall penetration factor $W_{f}$. 


\subsubsection{SINR of Macro Users}

Macro users are assigned the orthogonal channels sharing the same spectrum while the femto users use OFCDM for their channel assignment. Therefore, by utilizing the equations (4.2), (4.6) and (4.7), we can write the expression for average SINR of a macro user. We also include the channel gain, comprised of path loss component $d_{l m}^{-\alpha_{M}}$. SINR of macro user during the $j^{\text {th }}$ signal element is defined as:

$$
\gamma_{j}^{M}=\frac{P_{j}^{M}}{I_{j}^{M}+N_{j}^{M}}
$$

where,

$N_{j}^{M}=$ noise power present at the macro (M) user during the $j^{\text {th }}$ signal element, $P_{j}^{M}=$ received power of the desired signal during the $j^{\text {th }}$ signal element,

$I_{j}^{M}=$ is the total interference power receieved by the macro $(\mathrm{M})$ user during the $j^{\text {th }}$ signal element $=I_{j}^{M . F}+I_{j}^{M . M}$,

$I_{j}^{M . F}$ is interference from femto $(\mathrm{F})$ users sharing the same subcarrier as the macro (M) user, $I_{j}^{M . M}$ is the interference from other macro $(\mathrm{M})$ users. As macro users are assigned with orthogonal channels, $I_{j}^{M . M}=0$. Therefore, $I_{j}^{M}=I_{j}^{M . F}$.

Noise power $N_{j}^{M}$ is calculated as $N o$ and signal power $P_{j}^{M}$ as $\varepsilon_{b}\left(\alpha_{j, m}\right)^{2}$ is obtained by finding the variance of (4.16). Interference power $I_{j}^{M}$ is taken from (5.2).

SINR of an $\ell^{t h}$ macro user using the $m^{\text {th }}$ subcarrier from the group $y$ is given as:

$$
\gamma_{j, m}^{(\ell)}=\frac{\varepsilon_{b}\left(\alpha_{j, m}^{(\ell)}\right)^{2} d_{l m}^{-\alpha_{M}}}{\sum_{i=1}^{K_{y}} d_{i m}^{-\alpha_{F M}} W_{f} K_{y} N \varepsilon_{c} E\left[\left(\alpha_{y}\right)^{2}\right]+N_{o}}
$$

As BPSK modulation is used by the macro users, the probability of bit error for the $j^{\text {th }}$ bit is given as,

$$
P_{b j}^{(\ell)}=Q\left(\sqrt{2 \gamma_{j, m}^{(\ell)}}\right)
$$


From the above expression, we can see that the SINR is dependent on the fading gain for macro user $\ell$, the interference power on subcarrier $m$ and femto wall penetration loss $W_{f}$. This interference is from all the femto users sharing the same subcarrier present in the group $y$. 


\section{Chapter 6}

\section{Results and Discussion}

A Monte Carlo analysis is done to evaluate the BER performance of OFCDM femtocells operating within a OFDM macrocell by making use of SINR expressions derived in section 5.5. Firstly, the simulation setup and system parameters are presented. BER performance of the femto and macro users is evaluated under various channel loads and spreading factors. Afterwards, best spreading factors for the optimum BER performance of macrocell users, subject to different femtocell $\mathrm{Eb} / \mathrm{No}$, are noted and reported in the form of a table that can be used as a deployment guideline.

\subsection{Simulation Setup and Parameters}

For OFCDM femto users, total spreading factor used is 32 and it is constant; while the time $\left(N_{t}\right)$ and frequency $\left(N_{f}\right)$ spreading factors are variable. The effect (due to different number of subcarriers) of different time and frequency spreading factors for different channel loads is analyzed. We present different cases starting from $32 \times 1$ which means $N_{t}=32$ and it corresponds to OFDM alone in femtocells and no time spreading. The maximum time domain factor utilized in OFCDM is 16, with a processing gain of 2 in the frequency domain. This spreading factor is denoted as $16 \times 2$. Simulations are also performed with spreading factors of $8 \times 4,4 \times 8$ and $2 \times 8$. Finally, spreading factor of $1 \times 32$ is presented to show purely 


\begin{tabular}{|c|c|c|}
\hline Parameters & Macro Value & Femto Value \\
\hline \hline No. of Base Stations $(\mathrm{BS})$ & 1 & 16 \\
\hline No. of Users $(\mathrm{L}, \mathrm{K})$ & 16,64 & $16,32,64$ \\
\hline Coverage Area $\left(R_{m}, R_{f}\right)$ & $50 \mathrm{~m}$ & $5 \mathrm{~m}$ \\
\hline Wall Penetration Loss $\left(W_{m}, W_{f}\right)$ & 1 & $10^{-1}, 10^{-2}$ \\
\hline Path Loss Exponent $\left(\alpha^{M}, \alpha^{F}\right)$ & -4 & -2 \\
\hline Channel Bandwidth $(\mathrm{BW})$ & $20 \mathrm{MHz}$ & $20 \mathrm{MHz}$ \\
\hline Subcarriers $(\mathrm{M})$ & $64,128,256$ & $64,128,256$ \\
\hline Carrier Frequency $\left(f_{c}\right)$ & $5 \mathrm{GHz}$ & $5 \mathrm{GHz}$ \\
\hline Chip rate & NA & $3.2 \mu \mathrm{s}, 6.4 \mu \mathrm{s}, 12.8 \mu \mathrm{s}$ \\
\hline
\end{tabular}

Table 6.1: Simulation Parameters

the CDMA case in femtocells. Each of these configurations utilizes a total spreading factor of 32 to provide a suitable performance comparison. Femto wall penetration factor, $W_{f}$ is also taken, as 0.1 (or as $-10 \mathrm{~dB}$ ) to include the effect of indoor signal penetration loss. BER and SINR expressions derived in the last chapter are utilized to obtain the results. We analyze the system performance by using 64, 128 and 256 subcarriers. Simulation parameters are given in the Table 6.1. The hybrid network in Figure 5.1 is used in the simulation and users are distributed as shown there.

\subsection{Femto BER Femto Eb/No}

These graphs are obtained by utilizing 128 subcarriers, and femto and macro users are denoted with the letter 'F' and 'M', respectively. In Figures 6.1 and 6.2, average femto user $\mathrm{BER}$ is plotted as a function of its average $\mathrm{Eb} / \mathrm{No}$ under various spreading factors. BER was obtained using equation 5.11 through Monte Carlo simulation.

\subsubsection{Interference-Limited Case}

Interference-limited case (with $64 \mathrm{~F}$ and $64 \mathrm{M}$ ) is shown in Figure 6.1, where the number of both types of users is maximum (of 64 users) and channel noise has less pronounced effect on performance. We can easily see that spreading factor $16 \times 2$ produces the best results, 


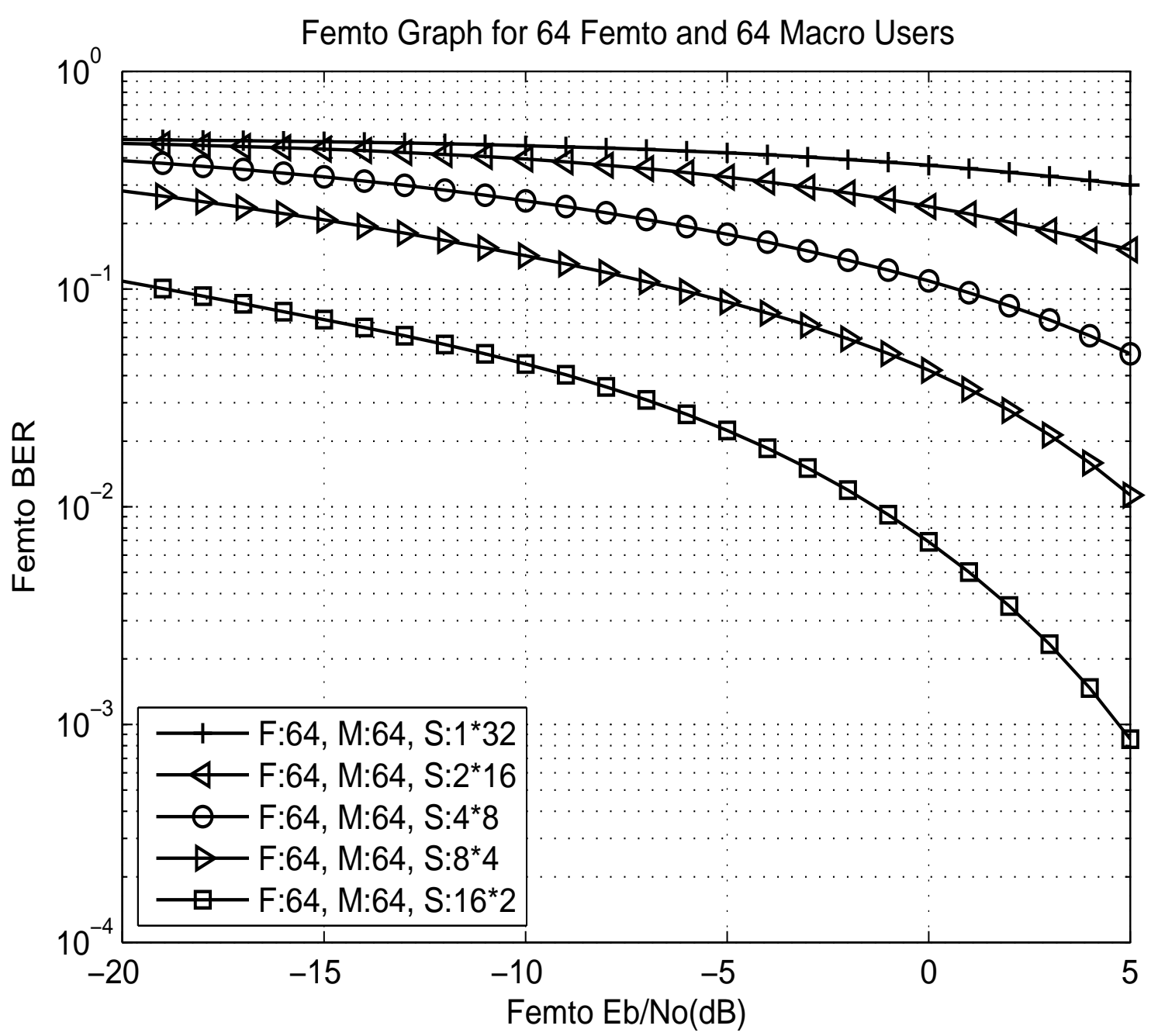

Figure 6.1: Femto BER in Interference Limited Case (64F-64M)

when time domain spreading is prioritized (i.e, $N_{t}=16$ ).

As for higher $N_{t}$, there are more available subcarrier groups which provide a better chance of locating subcarriers with the best fading characteristics. This results in minimizing MAI and maximizing the average BER. As the frequency domain is prioritized, performance degradation is observed, even though there is an increase in the frequency diversity, it is compromised by the increase in MAI. For example, we observe approximately $6 \mathrm{~dB}$ loss when going from $N_{f}=16$ to $N_{f}=8$ from the figure. 


\subsubsection{Noise-Limited Case}

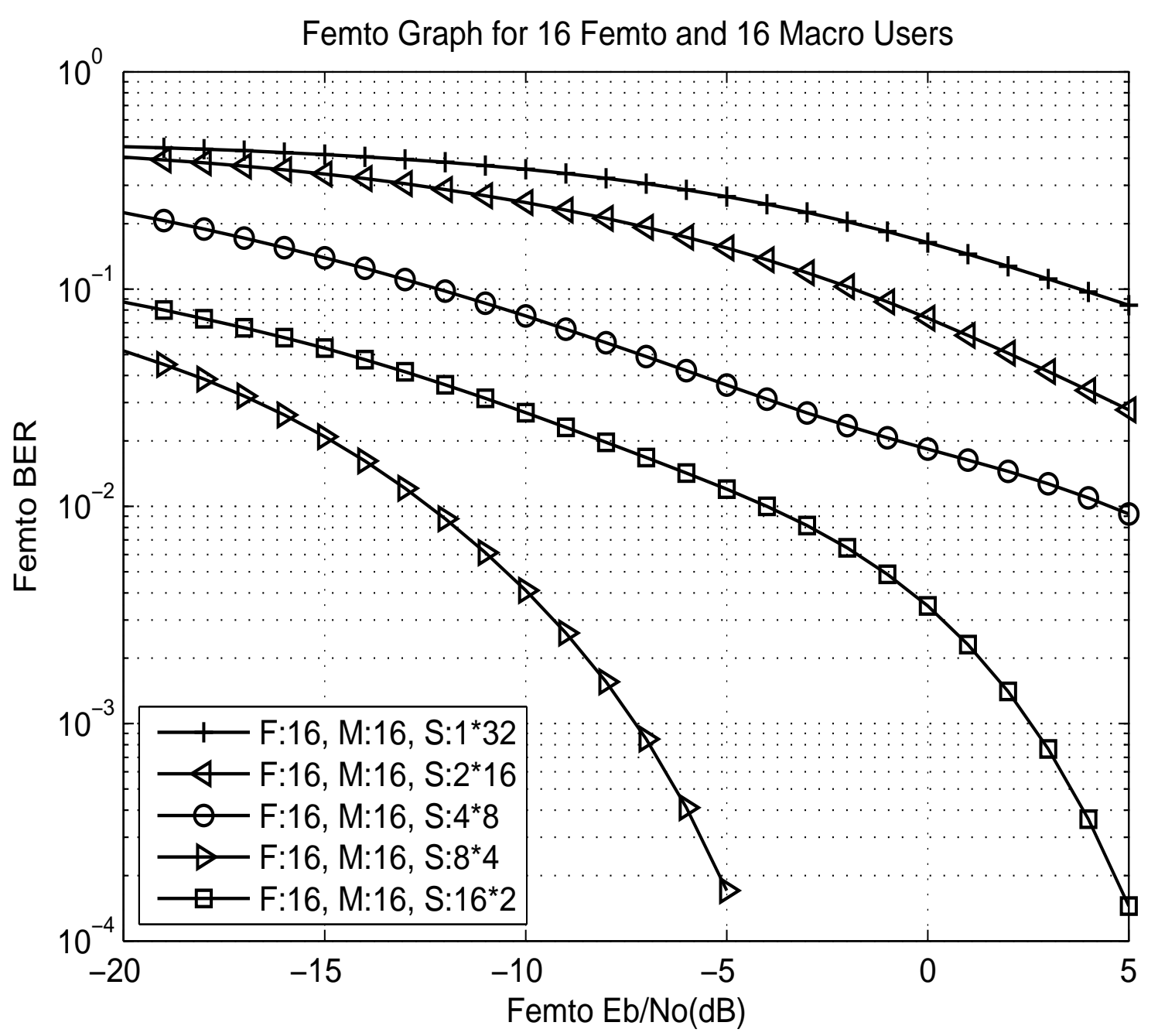

Figure 6.2: Femto BER in Noise Limited Case (16F-16M)

The results for noise-limited case (16F and $16 \mathrm{M})$ are reported in Figure 6.2, when number of femto as well macro users are very less and the ratio of noise power to the interference power is high. As the number of users is less so is the MAI. That is why better results are obtained when frequency spreading is prioritized. It is obvious from the graph that $8 \times 4$ spreading produces better result than $16 \times 2$ spreading. It provides BER of $10^{-2.5}$ compared to the spreading factor of $16 \times 2$ that provides BER of $10^{-1.6}$ at the same femto Eb/No of $-10 \mathrm{~dB}$. Its performance is further improved at higher values of Eb/No. 


\subsection{Macro BER Femto Eb/No}

We should analyze the macro BER as well to get the net effect of variable spreading in femtocells. In Figures 6.3 and 6.4, average macro user BER is plotted as an increasing function of average femto $\mathrm{Eb} / \mathrm{No}$ under various spreading factors.

\subsubsection{Interference-Limited Case}

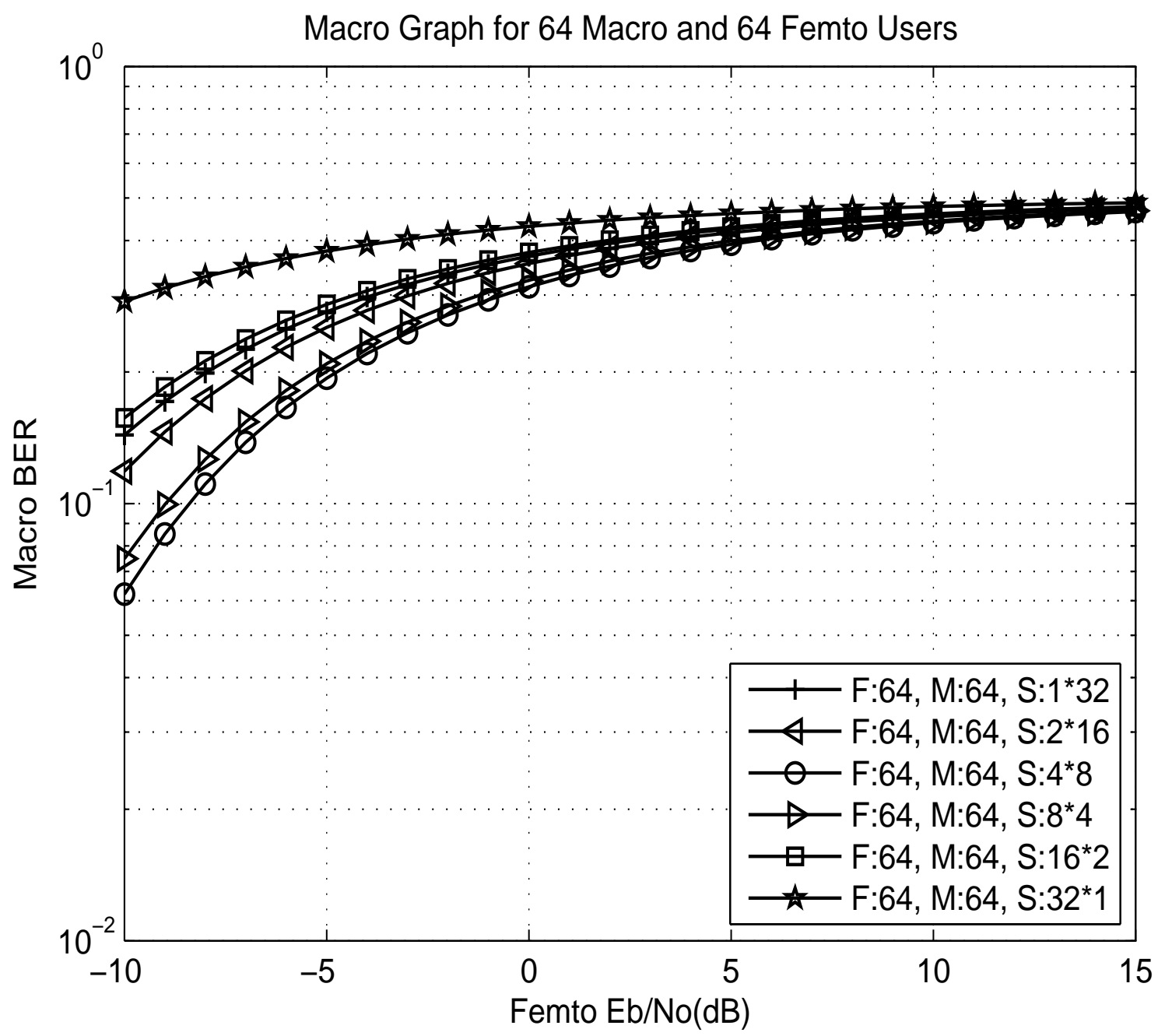

Figure 6.3: Macro BER vs. Femto Eb/No (64M-64F)

Figure 6.3 shows interference-limited case under various spreading for macro user BER. 
It is obvious from the figure that $4 \times 8$ spreading produces the best BER performance, when the channel load is maximum. As the channel is interference-limited and channel load is maximum, better BER results are obtained at high time domain spreading. These results are further supported by the plots in Figures 6.7 and 6.8. Above results are observed at low values of femto Eb/No while, at high Eb/No macro BER curves seem to be invariant. It explains that as the interference becomes stronger, different spreading factors tend to lose their influence on the macro BER.

\subsubsection{Noise-Limited Case}

It is evident from Figure 6.4 that macro users get better results when frequency domain spreading is prioritized. As the femto $\mathrm{Eb} / \mathrm{No}$ is increased, it increases the potential interference to the macro users thus decreasing the macro user BER. As the channel is noise-limited and channel load is minimum, better BER results are obtained at high frequency domain spreading. $2 \times 16$ proves to be better, for example as it provides BER of $10^{-2.9}$ compared to the spreading factor of $4 \times 8$ that provides $\mathrm{BER}$ of $10^{-1.8}$ at the same femto Eb/No of $-10 \mathrm{~dB}$.

\subsection{Mcaro BER Macro Eb/No}

A 3D plot is drawn to show the relationship between macro BER and macro Eb/No at various femto Eb/No, under different spreading factors in Figures 6.5 and 6.6 for interference-limited and noise-limited cases respectively. We are utilizing 3D plots as we have two parameters varied with respect to one fixed. Macro and femto BER are varied with respect to fixed desired femto Eb/No. Macro users are not using OFCDM directly, but the effect of spreading is reflected on it by the interference caused by the femto users (using OFCDM). Therefore, these curves are simply reflecting different levels of interference at different spreading factors. That exactly compliments the femto BER curves shown in Figures 6.1 and 6.2. 


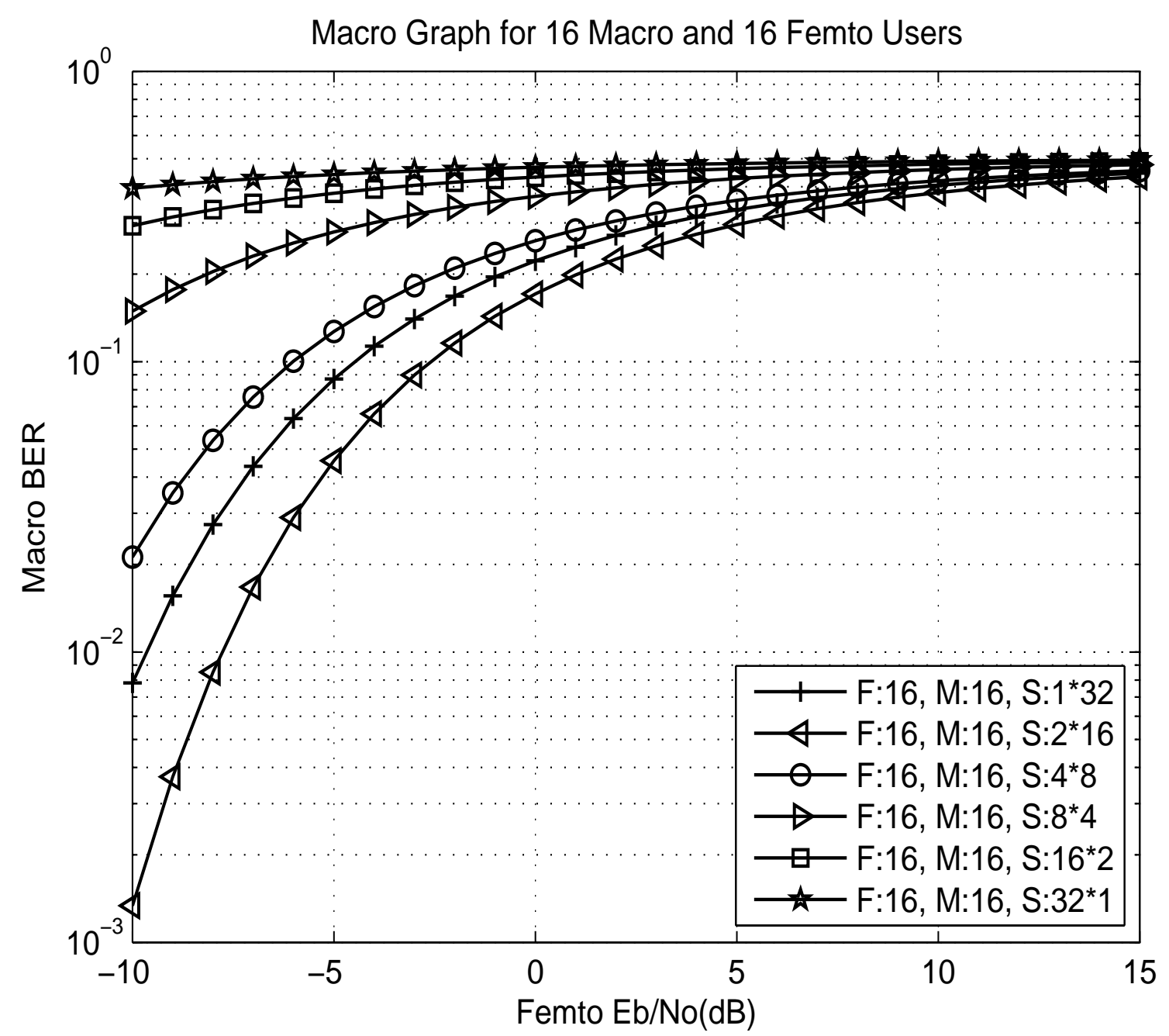

Figure 6.4: Macro BER vs. Femto Eb/No (16M-16F)

\subsubsection{Interference-Limited Case}

In Figure 6.5 interference-limited case is given, where number of users is maximum. As the number of users is maximum so is the MAI, thus high time spreading factor is required to produce better BER performance. $1 \times 32$ spreading curve shows worst BER performance due to excessive interference caused by both types of users. Therefore, when time spreading is prioritized, better results are observed as shown in $4 \times 8$ spreading curve. 


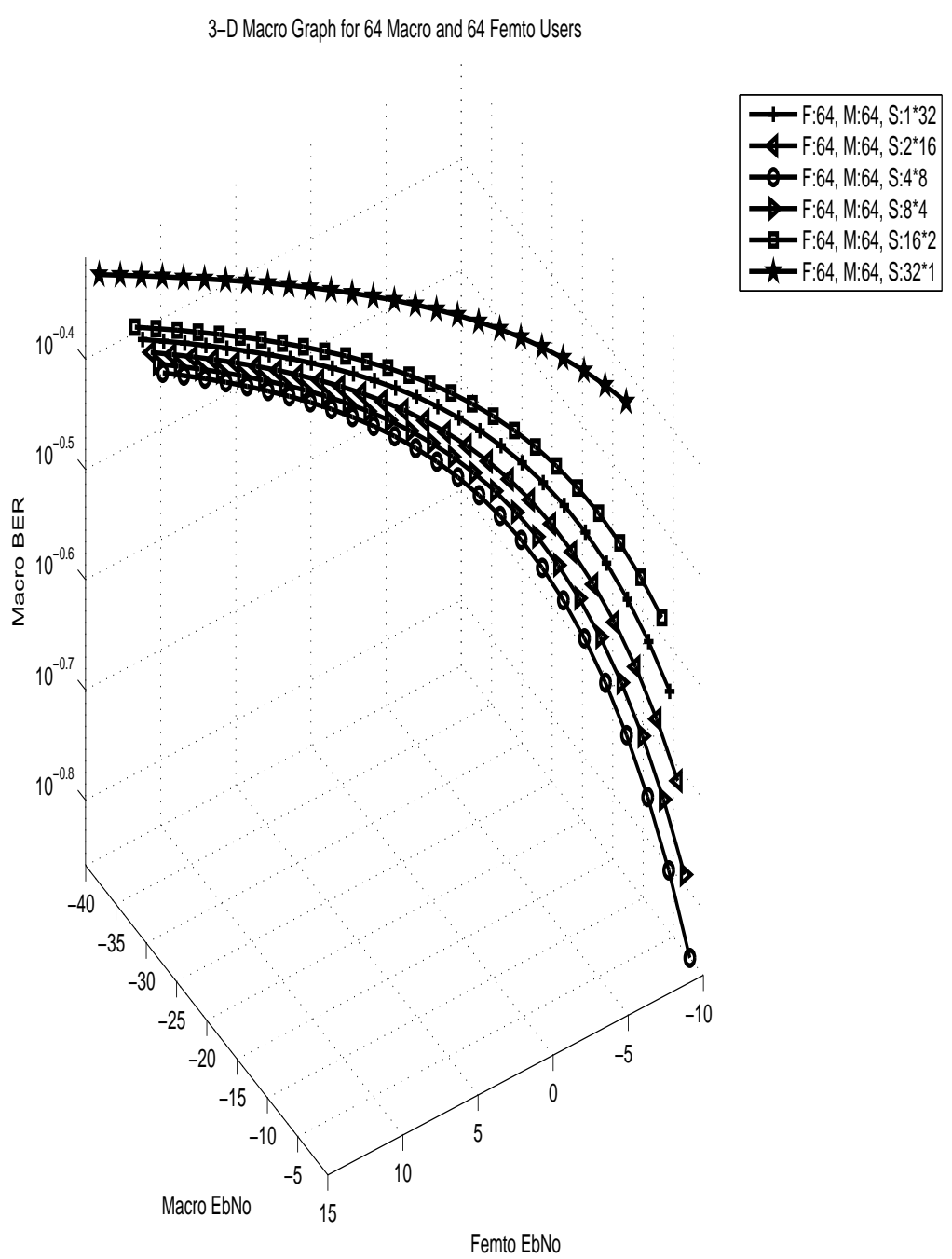

Figure 6.5: Macro BER in Interference Limited Case (64F-64M)

\subsubsection{Noise-Limited Case}

In Figure 6.6, the results for noise-limited case is observed, where number of users is minimum and channel noise takes the precedence. $1 \times 16$ and $2 \times 16$ spreading curves produce better results, when frequency domain spreading is prioritized.

It also shows that at lower femto Eb/No, same spreading factor can produce better BER characteristics than at higher values of Eb/No. That is explained as, stronger femto signal tends to interfere severely with the macro users thus decreasing its potential BER. There is no very significant change in BER floor as frequency diversity increases the signal power, 


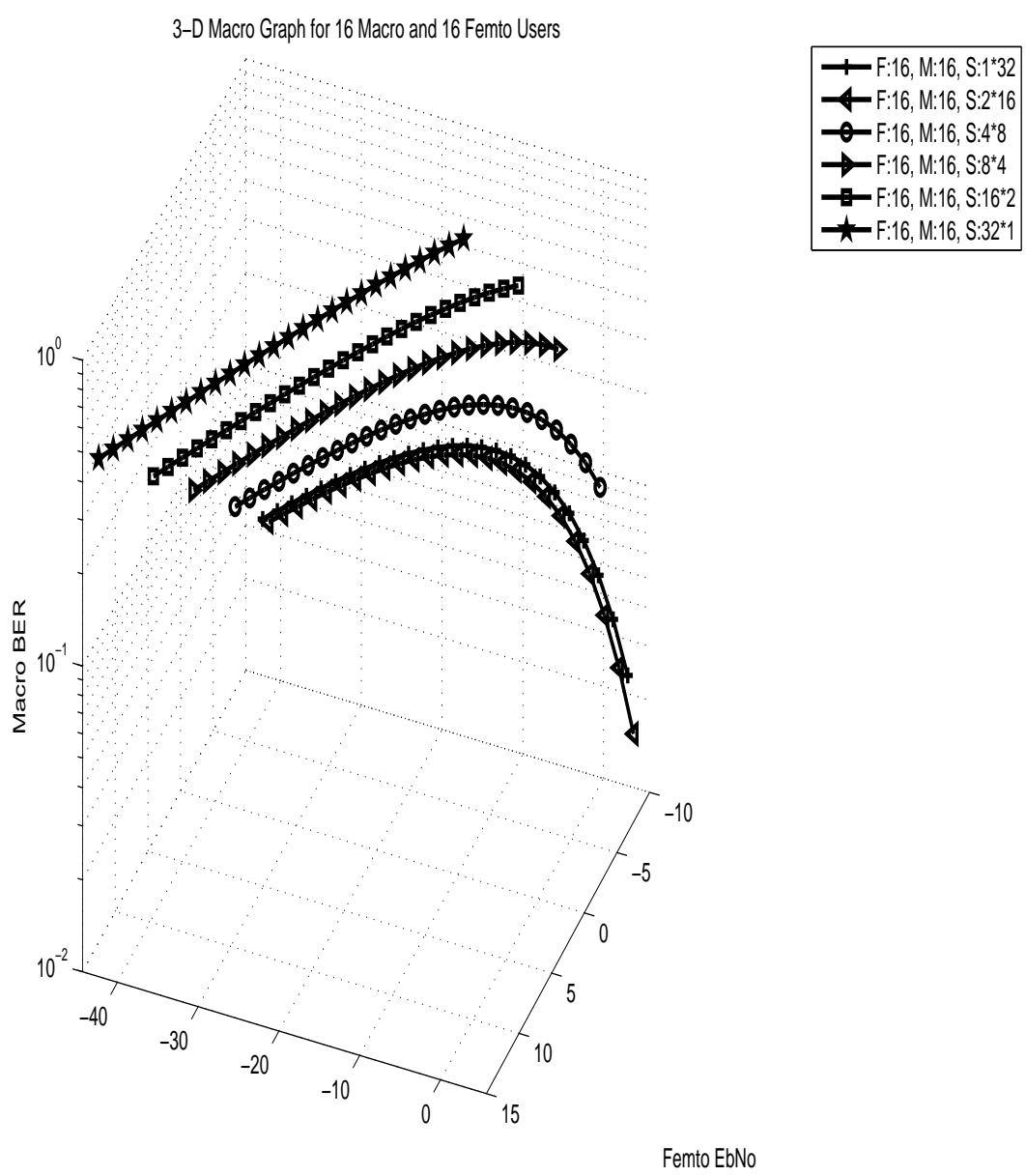

Macro EbNo

Figure 6.6: Macro BER in Noise Limited Case (16F-16M)

but the choice of subcarrier groups and subcarriers increases the interference power as well proportional to the signal power. Collectively speaking, these curves show the trend on how femto 2D spreading affects macro user Eb/No and in-turn its BER. 


\subsection{Optimal Spreading Factor}

The relationship between macro BER and femto spreading effect can be further investigated and elaborated as shown in Figures 6.7- 6.11 for interference-limited and noise-limited cases. Three curves are obtained at three femto Eb/No values of $-5 \mathrm{~dB},-7 \mathrm{~dB}$ and $-10 \mathrm{~dB}$. By plotting these curves, best spreading factor utilized by femto users, from macro performance perspective, is observed. This information will be useful in the efficient co-channel

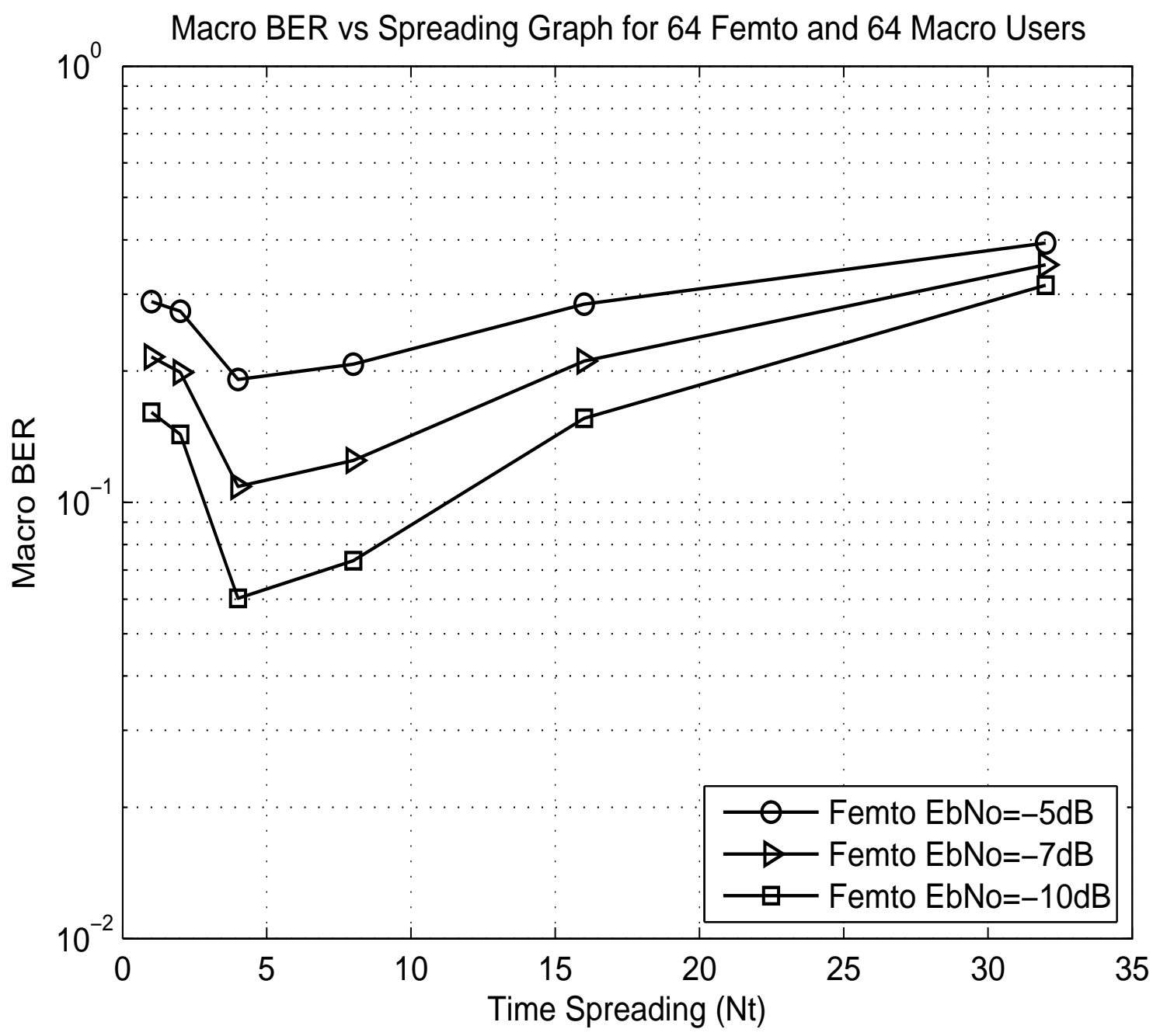

Figure 6.7: Macro BER vs. Femto Spreading in Interference Limited Case (64F-64M)

deployment of hybrid femto/macro users. As macro users are assigned with the orthogo- 
nal channels, the only source of interference is from co-channel femto users. Note that we fixed the total spreadings to 32 and hence, as time spreading increases, frequency spreading decreases.

\subsubsection{Interference-Limited Case}

Interference limited case is shown in Figure 6.7 and it shows the macro BER behavior when the number of femto users is set at maximum of 64 . By increasing the time domain spreading factor from 2 to 4 of femto users, improvement in the BER of macro users is observed. Further increase in time spreading deteriorates the macro user BER as shown in Figure 6.7.

By increasing the time domain spreading factor for femto users, MAI is reduced thus improving the BER of femto users, but at the same time there is a decrease in the frequency diversity which has a positive effect on the macro BER. As a result, the interference caused to the conflicting macro users is decreased, which improves the BER performance of macro users. This continues till $N_{t}=4$. At this point, macro user has the best BER achieved at the specific femto Eb/No $(-5 \mathrm{~dB},-7 \mathrm{~dB},-10 \mathrm{~dB})$ of femto users. Afterwards as the $N_{t}$ for femto user is further increased (to 8, 16, 32), we observe the degradation in macro BER. As femto user $N_{t}$ further increased, MAI is reduced for them thus improving their performance. But macro users are assigned orthogonal co-channels thus increasing their probability of interference with the conflicting femto users. Hence, the optimum choice of femto spreading factor, from macro perspective is $4 \times 8$ spreading in interference-limited scenarios. There is not much difference in terms of BER value between $-5 \mathrm{~dB}$ and $-10 \mathrm{~dB}$ curves as it is an interference-limited scenario and interference effect is dominant.

\subsubsection{Noise-Limited Case}

In Figure 6.8, the number of femto users is smaller and the system is more influenced by the channel noise than the cross or co-layer interference. Macro BER has best value when frequency spreading is prioritized. Therefore, the optimum choice of femto spreading factor, 


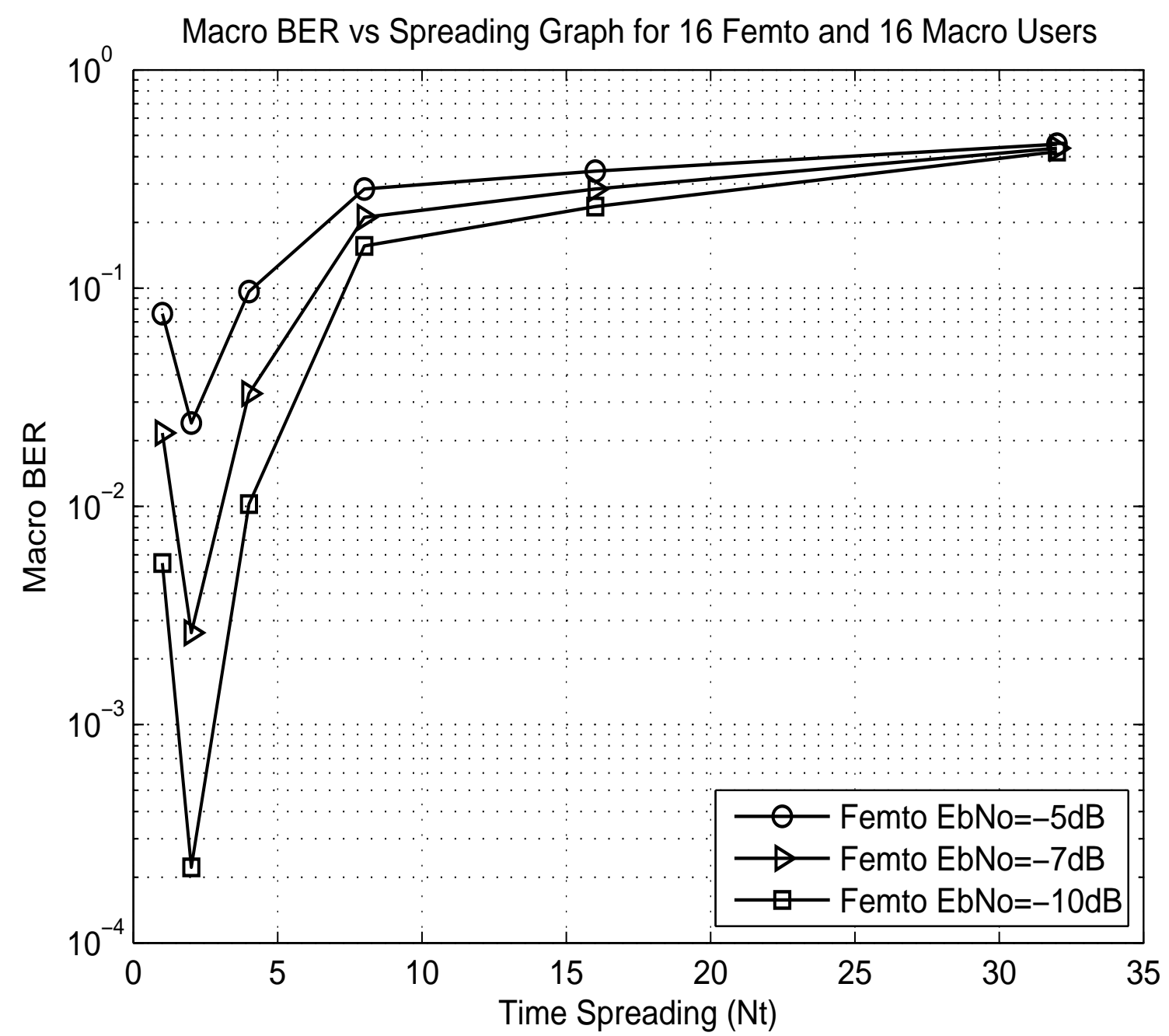

Figure 6.8: Macro BER vs. Femto Spreading in Noise Limited Case (16F-16M)

from macro perspective is $2 \times 16$ spreading in an noise-limited scenario. As the number of users is smaller and with increase in $N_{t}$, it will further reduce the MAI for femto user, thus improving their BER. But there is an adverse effect on the macro BER. There is a noticeable difference between the femto $\mathrm{Eb} / \mathrm{No}=-5 \mathrm{~dB}$ and $\mathrm{Eb} / \mathrm{No}=-10 \mathrm{~dB}$ curves. There is an improvement in the macro BER curve for Eb/No of $-5 \mathrm{~dB}$. Therefore, in the noise-limited region the strength of the interfering femto users has a dominant effect. There is little change in the macro BER from $N_{t}=16$ and $N_{t}=32$ of femto user, as decrease in MAI and corresponding decrease in diversity equalizes each other and produces the same 
effect on the interfering macro users and in turn in their BER. It is observed that switching the system from interference-limited to noise-limited, that is when the number of femto users increases from 16 to 64, actual change of macro BER characteristic takes place. This means that if macro user is showing good performance at high time domain spreading than at certain femto spreading, it changes its behavior and starts showing poor performance at high time spreading. The change of macro user from 16 to 64 does not affect the macro BER curve but has an effect on femto BER. That means by increasing the load (cross layer) in the channel, $4 \times 8$ spreading proves to be the best for macro system under investigation.

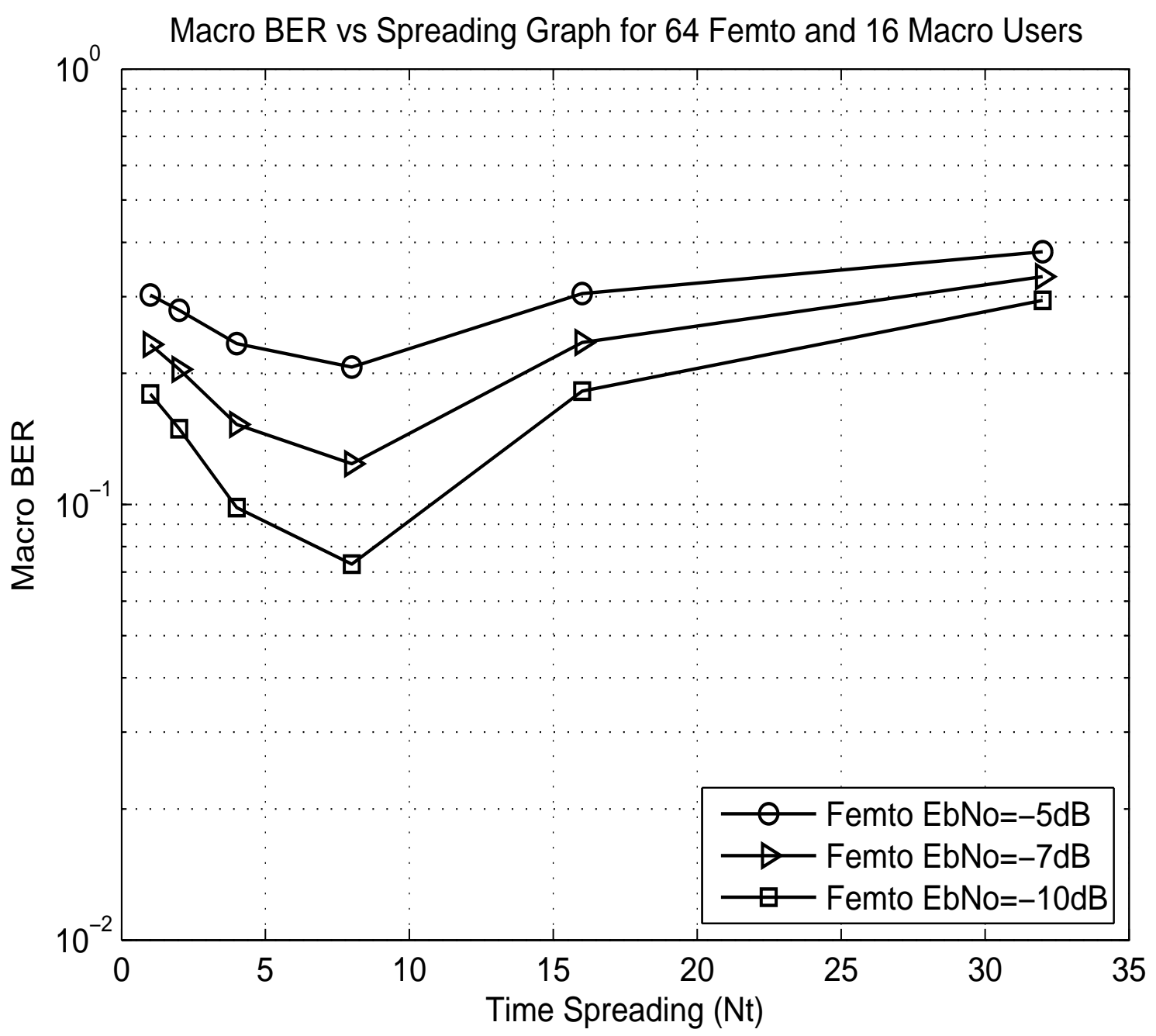

Figure 6.9: Macro BER vs. Femto Spreading (64F-16M) 


\subsubsection{Moderate Users Case}

We have so far studied the extreme operating cases in the interference and noise limited region for this hybrid macro/femto network. If we have maximum femto load but minimum macro load, we note that when time domain spreading is prioritized for macro users, it shows the best BER performance. In Figure 6.9 , spreading $8 \times 4$ shows to be the best spreading for 64 femto and 16 macro users case.

We also analyze the situation with moderate number of femto users for minimum and maximum macro channel utilization. In Figures 6.10 and 6.11, number of femto users is

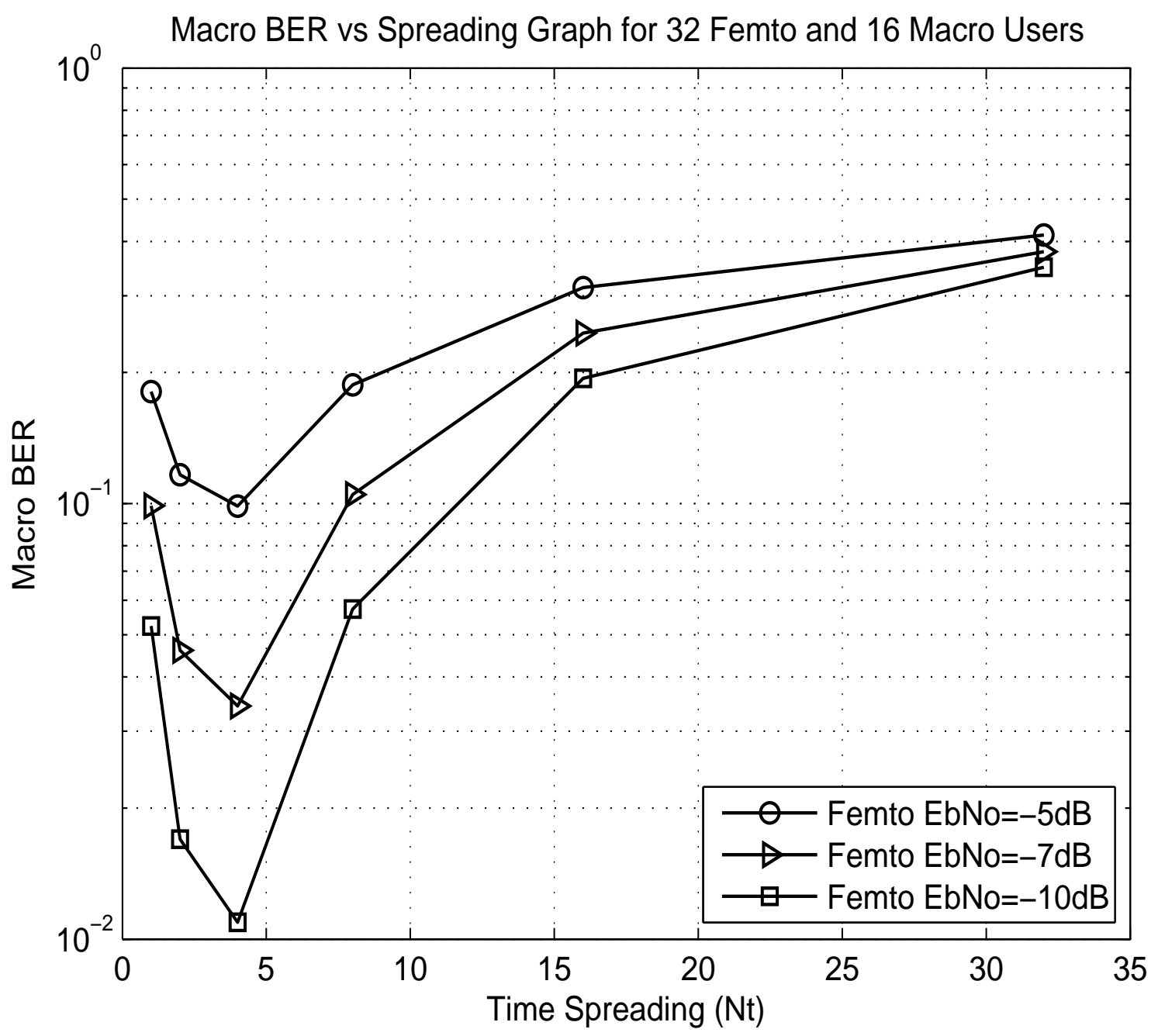

Figure 6.10: Macro BER vs. Femto Spreading (32F-16M) 


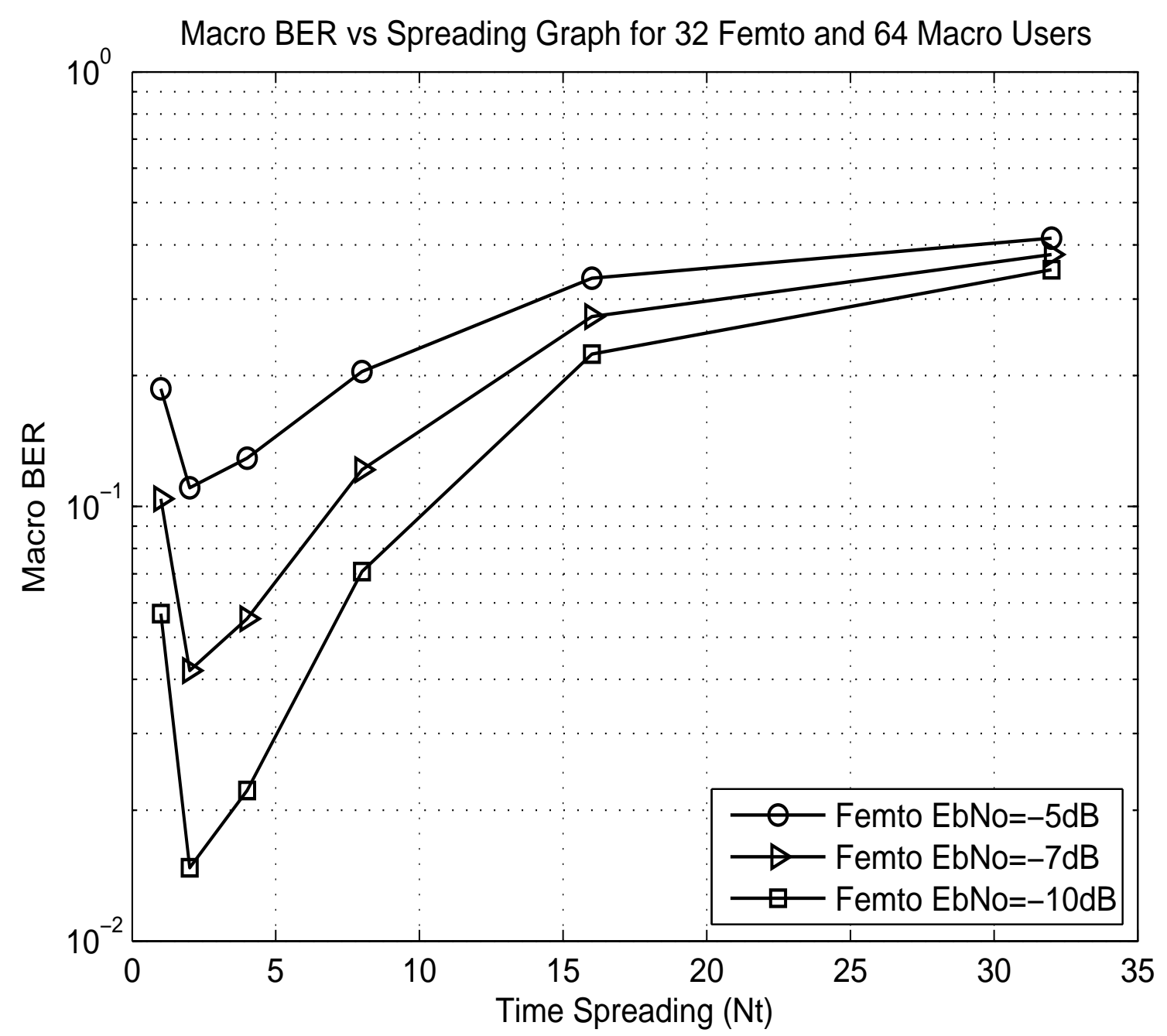

Figure 6.11: Macro BER vs. Femto Spreading (32F-64M)

32 that is a moderate load, while the numbers of macro users are 16 and 64 respectively. We will see that the optimum spreading factor from macro perspective is $4 \times 8$ for 16 and $2 \times 16$ for 64 macro users. This means that macro users show better BER performance when frequency domain spreading is prioritized as already observed. The change in the optimum spreading point with the change in the femto user is due to the different group assignment to all the femto users. As the number of subcarriers are fixed for each case and number of femto users is varying, the number of subcarrier groups assigned to each user will also change. This change in allocation of subcarriers has not only a direct impact on BER of femto users but 
also an indirect impact on macro users due to variation in cross-layer interference.

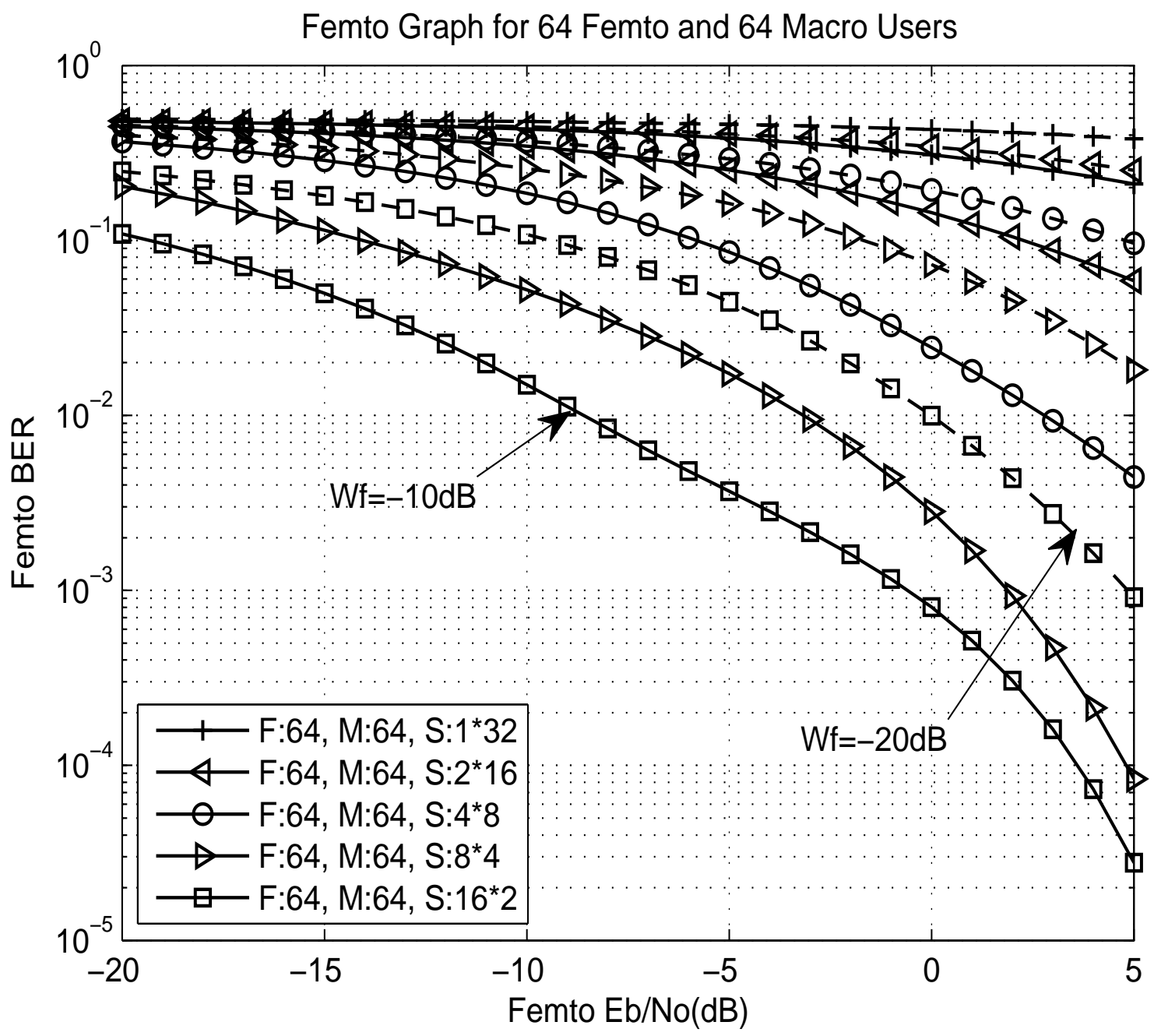

Figure 6.12: Femto BER in Interference Limited Case (64F-64M) for Various $W_{f}$

\subsection{Femto Wall Penetration}

We will analyze the effect of femto wall penetration, on the femto BER and macro optimal spreading as explained with the plots obtained in the section 6.2 and 6.5 respectively. We have used two values of femto wall penetration factor, i.e, $W_{f}=(-10 \mathrm{~dB}$ and $-20 \mathrm{~dB})$. 


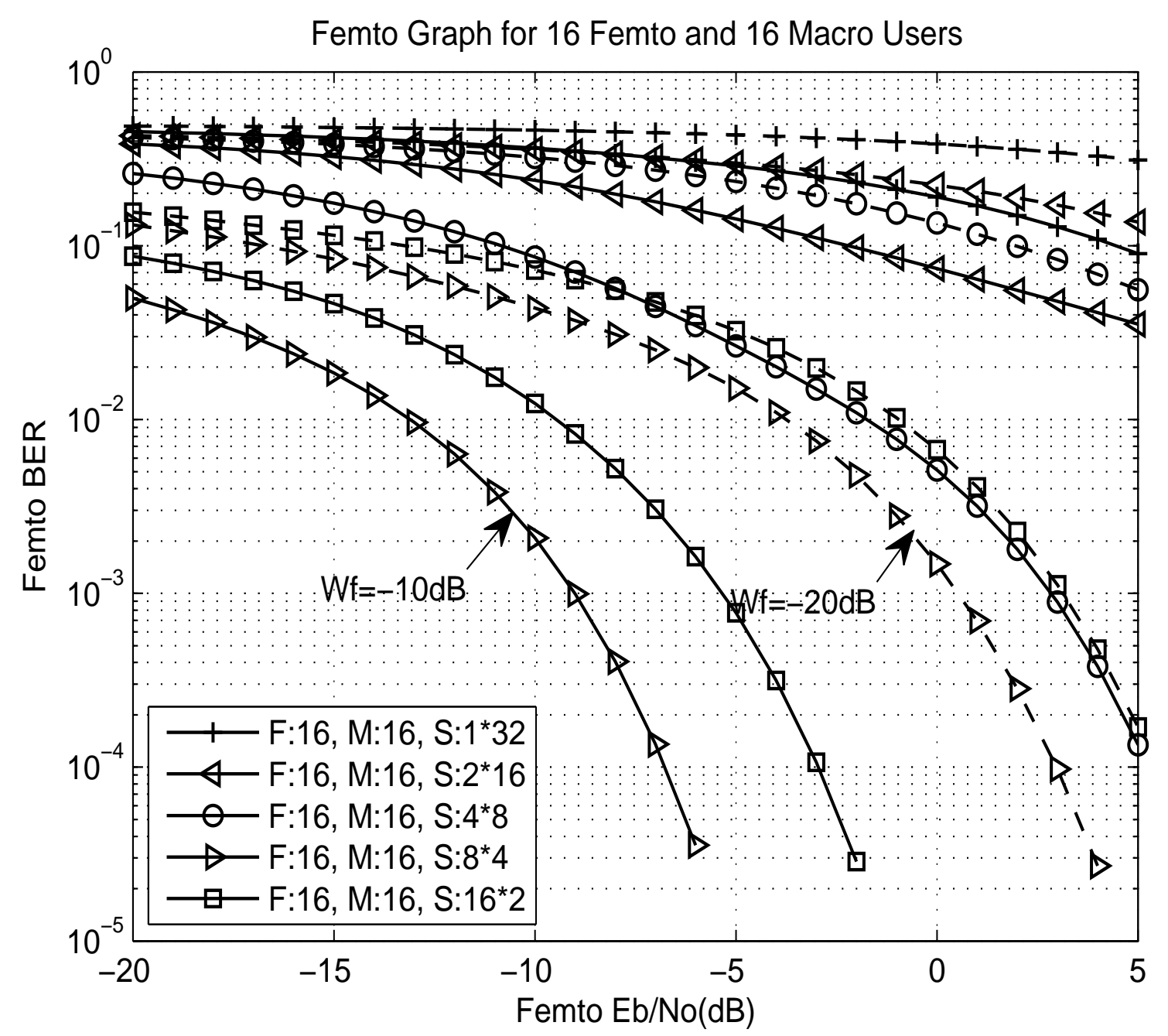

Figure 6.13: Femto BER in Noise Limited Case (16F-16M) for Various $W_{f}$

\subsubsection{Femto BER}

Interference-limited case is analyzed in Figure 6.12, in which spreading curves with $W_{f}=-$ $10 \mathrm{~dB}$ shows better results than with $W_{f}=-20 \mathrm{~dB}$. That can be explained as the received signal is less stronger with the high wall penetration factor and hence femto user shows poor BER performance at $W_{f}=-20 \mathrm{~dB}$. The same response continues for the noise-limited scenario, as shown in Figure 6.13. Increase in the $W_{f}$ will reduce the signal strength, thus degradation in BER is observed for femto users.

With the increase in $W_{f}$, cross-layer interference from macro to femto users is decreased 
and thus improvement in femto BER is expected. We do not see this effect, as degradation in the femto signal is much stronger with the increase in $W_{f}$. Hence, the effect of decrease in the cross-layer interference is suppressed by the effect of femto signal deterioration.

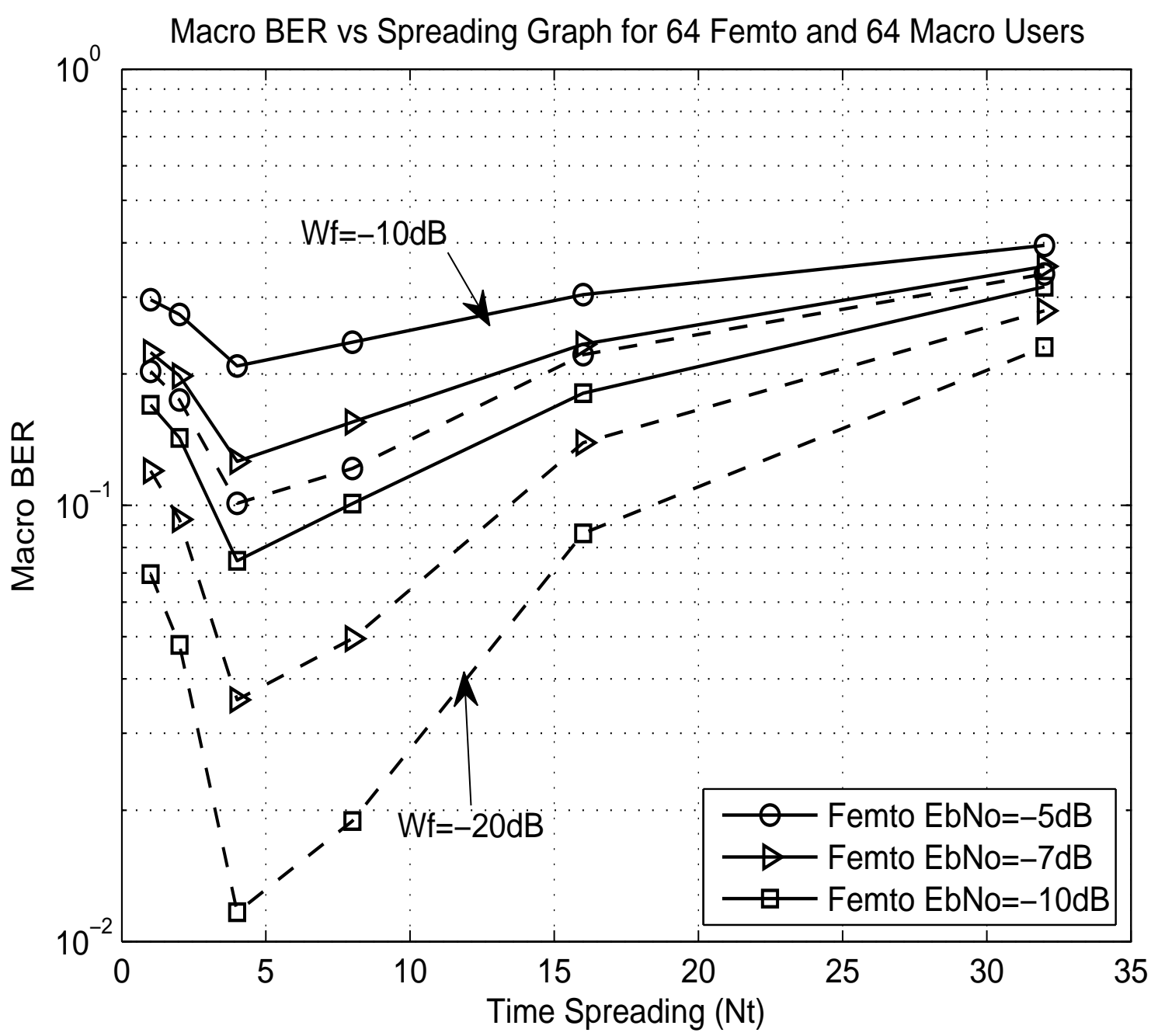

Figure 6.14: Macro BER vs. Femto Spreading in Interference Limited Case (64F-64M) for Various $W_{f}$

\subsubsection{Macro BER}

Macro optimal spreading for interference-limited case is shown in Figure 6.14. It is obvious that curves with $W_{f}=-10 \mathrm{~dB}$ show better performance than with $W_{f}=-20 \mathrm{~dB}$. As the increase 


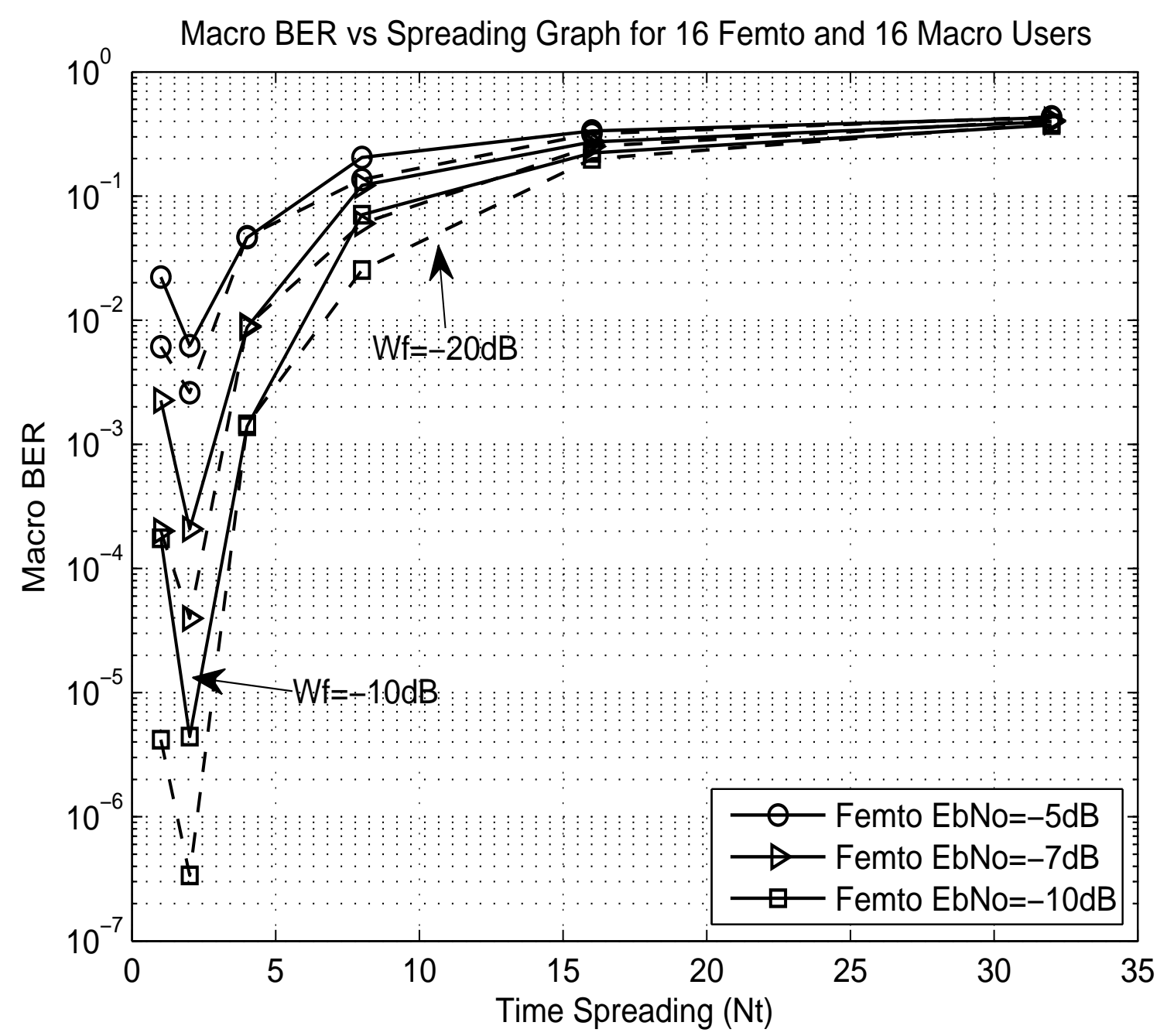

Figure 6.15: Macro BER vs. Femto Spreading in Noise Limited Case (16F-16M) for Various $W_{f}$

in the wall penetration factor for femto users, there is the decrease in the potential interference faced by the macro users (who experience co-channel interference from femto users only) and as a result there is the improvement in their BER. There is a noticeable difference between the curves for two $W_{f}^{\prime} s$. As number of users are maximum and decrease in the strength of their signals will reduce the interference caused to macro user considerably, thus improving their BER. Secondly, we can see that the optimal point does not change with the change in femto $W_{f}$ and remains at $4 \times 8$ in this case. 


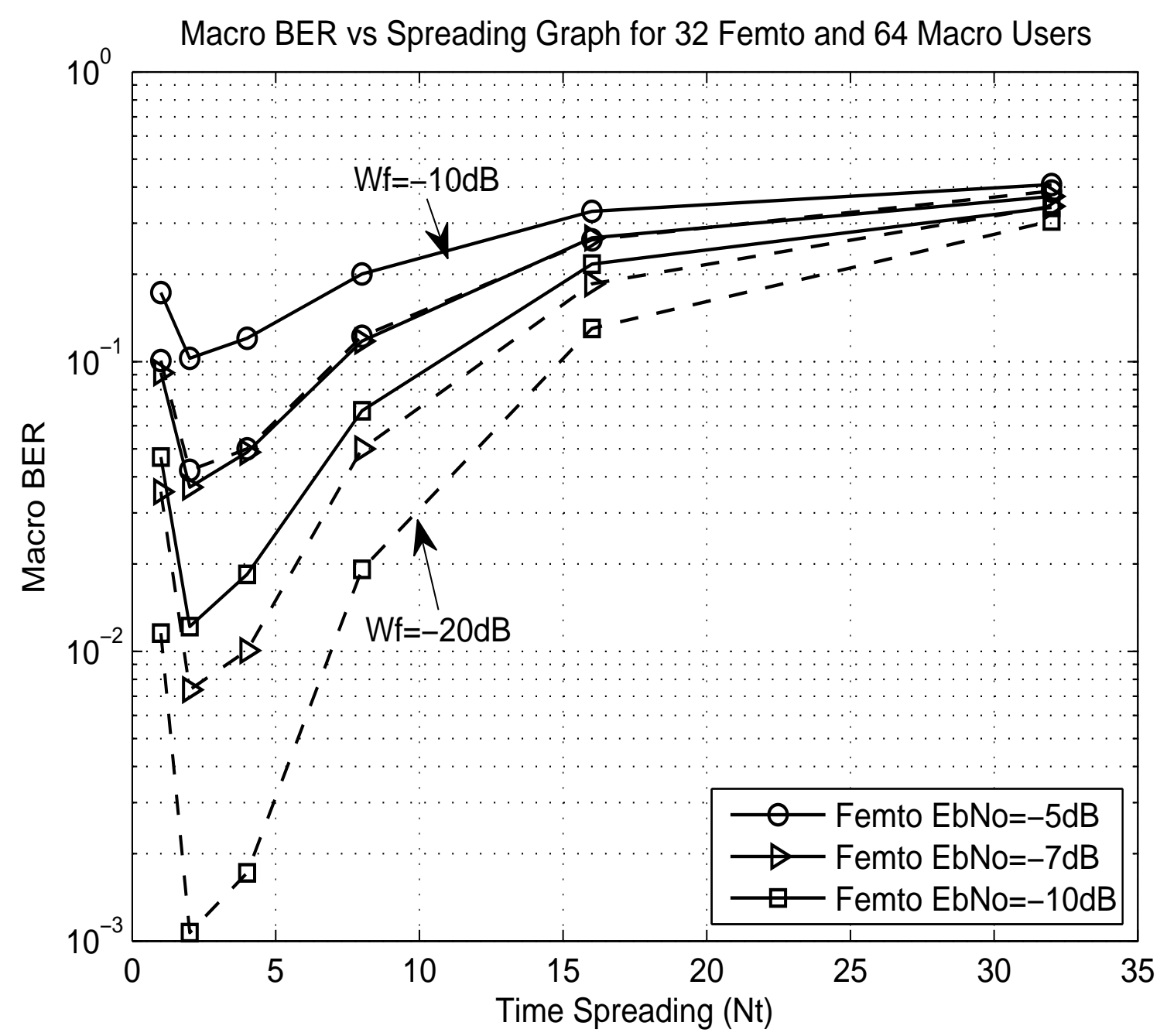

Figure 6.16: Macro BER vs. Femto Spreading (32F-64M) for Various $W_{f}$

Figure 6.15 shows the effect of $W_{f}$ on the macro optimal spreading in noise-limited case. Curves for $W_{f}=-20 \mathrm{~dB}$ produces better results than with $W_{f}=-10 \mathrm{~dB}$, as number of users is less and so is the cross layer interference. High femto wall penetration will further reduce the interference thus improving the BER performance for macro users.

In Figures 6.16 and 6.17, effect of $W_{f}$ is further investigated for different combinations of femto and macro users. Better curves are obtained for $W_{f}=-20 \mathrm{~dB}$ than for $W_{f}=-10 \mathrm{~dB}$ due to obvious reasons, as already explained. There is no noticeable difference between different $W_{f}$ for $(32 \mathrm{~F}-16 \mathrm{M})$ case compared to the prominent improvement for $(64 \mathrm{~F}-16 \mathrm{M})$ scenario, as 
in the latter case number of femto users is maximum and increase in femto $W_{f}$ will improve the macro user BER considerably.

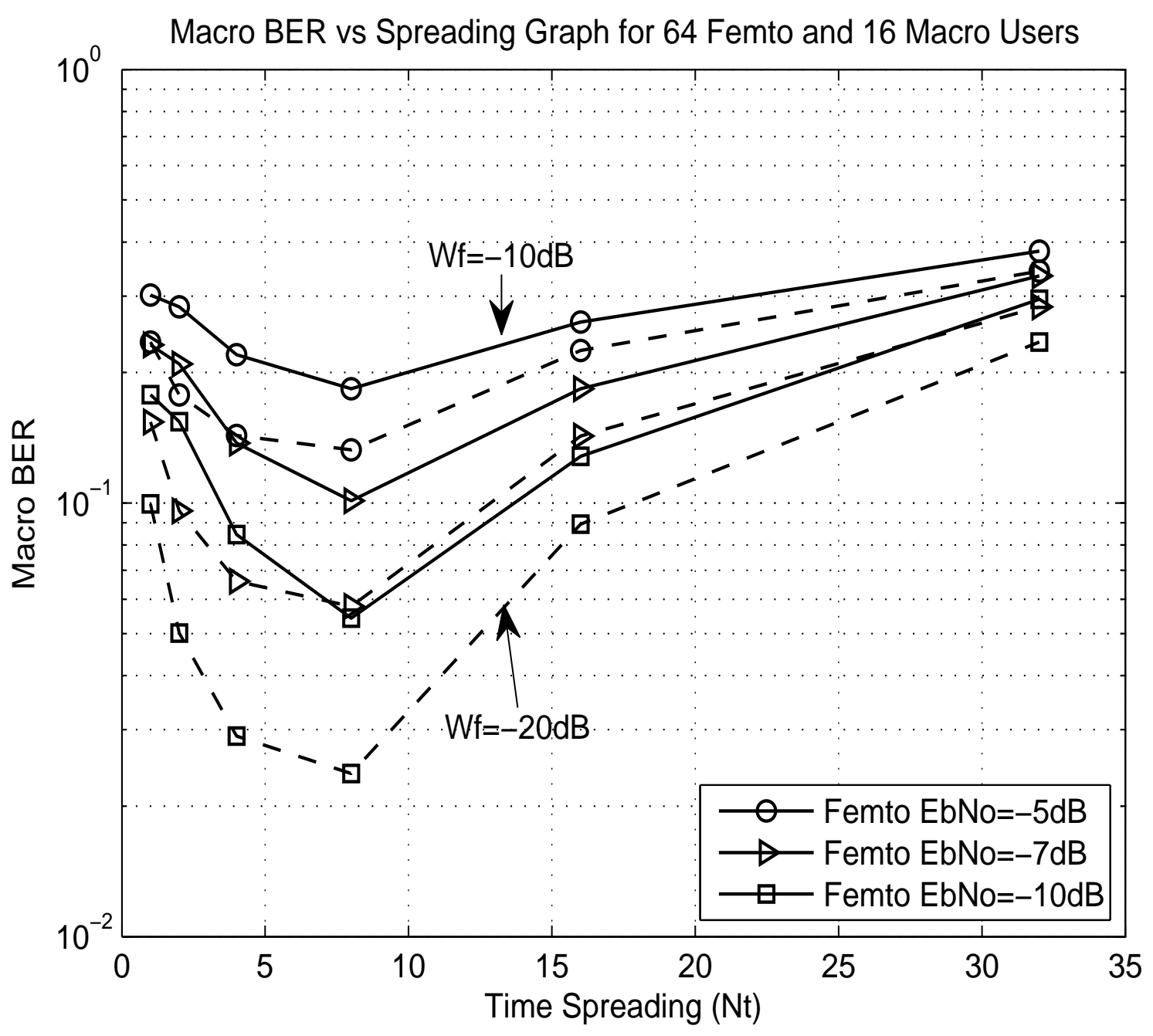

Figure 6.17: Macro BER vs. Femto Spreading (64F-16M) for Various $W_{f}$ 


\subsection{Optimal Spreading at Various Channel Loads}

In Table 6.2, 6.3 and 6.4, previously obtained results are summarized for 64, 128 and 256 subcarriers respectively for $W_{f}=-10 \mathrm{~dB}$. Note that femto wall penetration factor does not change the optimal spreading factor, as noted earlier. It gives an insight about the best spreading in frequency $\left(N_{f}\right)$ and time $\left(N_{t}\right)$ domain for specific channel loads.

\begin{tabular}{|l|l|l|l|}
\hline Scenario & $\begin{array}{l}\text { Femto } \\
\text { Users }\end{array}$ & $\begin{array}{l}\text { Macro } \\
\text { Users }\end{array}$ & $\begin{array}{l}\text { Optimum } \\
\text { Spreading } \\
\left(N_{t} \times N_{f}\right)\end{array}$ \\
\hline \hline Noise-limited & 16 & 16 & $2 \times 16$ \\
\hline Moderate Femto, Low Macro & 32 & 16 & $4 \times 8$ \\
\hline High Femto, Low Macro & 64 & 16 & $8 \times 4$ \\
\hline \hline Low Femto, High Macro & 16 & 64 & $2 \times 16$ \\
\hline Moderate Femto, High Macro & 32 & 64 & $4 \times 8$ \\
\hline Interfernce-limited & 64 & 64 & $8 \times 4$ \\
\hline
\end{tabular}

Table 6.2: Optimum Spreading Factor for Various Channel Loads for 64 Subcarriers

\begin{tabular}{|l|l|l|l|}
\hline Scenario & $\begin{array}{l}\text { Femto } \\
\text { Users }\end{array}$ & $\begin{array}{l}\text { Macro } \\
\text { Users }\end{array}$ & $\begin{array}{l}\text { Optimum } \\
\text { Spreading } \\
\left(N_{t} \times N_{f}\right)\end{array}$ \\
\hline \hline Noise-limited & 16 & 16 & $2 \times 16$ \\
\hline Moderate Femto, Low Macro & 32 & 16 & $4 \times 8$ \\
\hline High Femto, Low Macro & 64 & 16 & $8 \times 4$ \\
\hline \hline Low Femto, High Macro & 16 & 64 & $1 \times 32$ \\
\hline Moderate Femto, High Macro & 32 & 64 & $2 \times 16$ \\
\hline Interference-limited & 64 & 64 & $4 \times 8$ \\
\hline
\end{tabular}

Table 6.3: Optimum Spreading Factor for Various Channel Loads for 128 Subcarriers

From Tables 6.2 and 6.4, it is evident that when number of subcarriers is low or high, the increase in the macro users does not effect the choice of optimum spreading. As the number of femto users change from 16 to 64 , a optimum spreading changes from $2 \times 16$ to $8 \times 4$ for 64 subcarriers as shown in Table 6.2. Increase in the number of macro users from 16 to 64 has a negligible affect. The same kind of behavior is observed in Table 6.4 for 256 


\begin{tabular}{|l|l|l|l|}
\hline Scenario & $\begin{array}{l}\text { Femto } \\
\text { Users }\end{array}$ & $\begin{array}{l}\text { Macro } \\
\text { Users }\end{array}$ & $\begin{array}{l}\text { Optimum } \\
\text { Spreading } \\
\left(N_{t} \times N_{f}\right)\end{array}$ \\
\hline \hline Noise-limited & 16 & 16 & $1 \times 32$ \\
\hline Moderate Femto, Low Macro & 32 & 16 & $2 \times 16$ \\
\hline High Femto, Low Macro & 64 & 16 & $2 \times 16$ \\
\hline \hline Low Femto, High Macro & 16 & 64 & $1 \times 32$ \\
\hline Moderate Femto, High Macro & 32 & 64 & $2 \times 16$ \\
\hline Interfernce-limited & 64 & 64 & $2 \times 16$ \\
\hline
\end{tabular}

Table 6.4: Optimum Spreading Factor for Various Channel Loads for 256 Subcarriers

subcarriers. It is also noted that for moderate femto users, the optimum spreading is $4 \times 8$ regardless of macro load. In Table 6.3, optimum spreading has been shown for moderate number of subcarriers of 128. It is obvious that change in the number of macro users has an impact on the choice of optimum spreading factor. As the number of macro users increases from 16 to 64 , optimum spreading factor changes from $1 \times 32$ to $4 \times 8$.

It is also observed that smaller number of subcarriers require high time spreading factor and larger number require high frequency spreading factor. This can be explained as, when the number of subcarriers is high, larger number of groups are available for the users to choose from; but if the number is low, lower number of groups is present thus increasing the MAI. Therefore, high time spreading should be employed to maximize the absolute performance by reducing MAI, and utilizing larger number of groups having lower number of subcarrier in it.

Key findings from the above research are summarized below:

- 2D spreading should be optimized according to the number of subcarriers, noise-limited and interference-limited systems and femto/macro loads.

- Interference-limited system favors increased time spreading especially when number of subcarriers is limited and noise-limited system favors increased frequency domain spreading. 
- When large number of subcarriers are available, optimum spreading favors increased frequency domain spreading regardless of the femto-macro loads, or whether operating envirement is noise or interference limited.

- Once the optimum spreading factor is determined, increase or decrease in the femto $\mathrm{Eb} /$ No doesn't matter. In all the plots in section 6.5, optimum spreading point remains the same for femto Eb/No of $5 \mathrm{~dB}, 7 \mathrm{~dB}$ and $10 \mathrm{~dB}$, that is independent of femto Eb/No.

- Femto wall penetration factor effects the femto BER directly. With its increase, femto signal strength is decreased thus reducing the potential interference faced by macro UEs. As a result macro BER is improved, but the choice of optimal spreading factor for macro UEs remain unaffected with the variation in femto wall penetration loss. 


\section{Chapter 7}

\section{Conclusions and Future Work}

\subsection{Summary}

Now-a-days, very important trend concerning cellular networks operating in licensed spectrum have emerged. The cellular operators are considering femtocells deployed in indoor environments such as homes and enterprise buildings as a complement to macrocells for the purposes of enhancing capacity and coverage indoors. Femtocells, both enhance and interfere, with the macro cellular network in ways that are not yet well fully understood. As broadband wireless access gains adoption and media-rich end user devices such as iPhones proliferate, the next frontier will be ultra-broadband wireless access which inevitably needs more spectrum. As end users often spend more time indoors, femtocells may be the first user equipment to offer such ultra-broadband access. Since these media-rich broadband applications require large bandwidth, OFCDM is a viable option to support the demanding requirements of these services. This scheme accommodates larger bandwidth by employing several narrowband subcarriers that are inherently resistant to ISI.

If we employ OFCDM in femtocells, we can provide a flexible cellular solution for the present day demand of media-rich applications. This system can flexibly mould itself, according to the varying channel conditions, in terms of time or frequency domain spreading. 
4G systems are already utilizing OFDMA as their underlaying technology and hence our assumed macro system does not require any switching of technology. When we smoothly integrate OFCDM femtocells into it, we can benefit from both. The time and frequency domain spreading employed by this system allows it to achieve higher performance than systems that employ one dimensional spreading alone. The frequency domain spreading factor can be increased to maximize gains from frequency diversity, while multipath diversity and minimal MAI can be provided with time domain spreading. The utility and benefits of OFCDM had already been shown in the literature. Therefore, keeping this in mind, we analyzed the effect of OFCDM technology over existing OFDMA macro cellular network.

We provided various graphs showing the impact of femto user spreading factor on the macro user BER. We noted that under maximum load conditions, $4 \times 8$ spreading (time $\times$ freq.) proves to be the best for macro users; and for the noise limited case, when there are limited number of users and channel noise is dominant, $2 \times 32$ spreading is the optimum choice for the considered hybrid cellular network configuration. These results are also supported by others in [26], [27], [48] and [51] in which it has been proved for traditional cellular system using OFCDM, that a high frequency domain spreading factor is superior under low channel loads because frequency diversity is achieved, while MAI remains small as few users access the channel simultaneously. Also if there is a large number of users, time domain spreading should be prioritized to minimize MAI. Our research and findings extended the existing knowledge to femtocells.

Our results proves the usefulness of OFCDM in femtocells as well. In fact, we extended the previous work in femto/macro hybrid network with the addition of optimum two dimensional spreading factor analytical derivation and validation through numerical simulations for different channel loads.

In addition to above, we have taken into account femto wall penetration loss, that is the important parameter to evaluate the femto performance. We evaluated the effect of femto wall penetration on macro BER for various spreading factors. It directly effects the femto 
and indirectly the macro BER. But the choice of optimal spreading factor for macro UEs remains unaffected with the variation in femto wall penetration loss.

\subsection{Future Research}

The important results obtained in this report bring several new ideas that could further resolve the issues in efficient deployment of femtocells. Some future directions on this topic are:

- By having a knowledge of best spreading combination as per macro user BER performance, a spreading factor can be changed based on the channel load conditions that exist in the cell without significantly changing the femtocell performance. This flexibility of spreading factor is the characteristic of OFCDM system. Therefore, with the change in the macro load, the femtocells and in turn femto users can change their $N_{t}$ and $N_{f}$ spreading factors keeping the total spreading to be constant.

- OFCDM femtocells can be converted to OFDM or CDMA alone femtocells according to the channel conditions such as delay spread, background noise, Doppler effect and different forms of interferences, by utilizing the natural flexibility in OFCDM technology. This adaptability of femto users according to the situations proves them to be a viable option for the future high speed broadband services.

- From femto BER performance angle, an adaptive algorithm can be developed to assign subcarrier groups, to femto users, having best SINR characteristics, thus improving the performance of femto users for given channel conditions.

- An interference management algorithm can be developed to avoid the potential crosslayer interference among femto and macro users. Femto users are assigned the available subcarrier groups and afterwards, they avoid using those groups that had been already 
utilized by macro users. This can improve the performance of both the femto as well macro systems significantly.

- We can utilize the OFCDM for macro and OFDM for femto users, thus investigating another paradigm in this regard. The effect of macro spreading on femto BER can also be investigated and reported. 


\section{Bibliography}

[1] Femto Forum, http://www.femtoforum.org/.

[2] J. Zhang and G. de la Roche, Femtocells: Technologies and Deployment. John Wiley and Sons, 2010.

[3] K. R. Santhi, V. K. Srivastava, G. SenthilKumaran, and A. Butare., "Goals Of True Broad bands Wireless Next Wave (4G-5G)," in IEEE Vehicular Technology Conference, vol. 4, pp. 2317-2321, October 2003.

[4] S.Hui and K.Yeung, "Challenges in the Migration to 4G Mobile Systems," IEEE Communications Magazine, vol. 41, pp. 54-59, December 2003.

[5] T. Miki, T. Ohya, H. Yoshino, and N. Umeda, "The Overview of the 4th Generation Mobile Communication System," in International Conference on Information, Communications and Signal Processing, pp. 1551-1555, December 2005.

[6] K. Tachikawa, "A Perspective on the Evolution of Mobile Communications," IEEE Communications Magazine, vol. 41, pp. 66-73, October 2003.

[7] V. DaSilva and E. S. Sousa, "Performance of Orthogonal CDMA Codes for QuasiSynchronous Communication Systems," in IEEE International Conference on Universal Personal Communications, vol. 2, pp. 995-999, October 1993. 
[8] L. Xiao and Q. Liang, "A Novel MC-2D-CDMA Communication System and Its Detection Methods," in IEEE International Conference on Communications, vol. 3, pp. 12231227, June 2000.

[9] H. Atarashi, S. Abeta, and M. Sawahashi, "Variable Spreading Factor Orthogonal Frequency and Code Division Multiplexing (VSF-OFCDM) for Broadband Packet Wireless Access," IEICE Transactions on Communications, vol. E86-B, pp. 291-299, January 2003.

[10] V. Chandrasekhar and J. G. Andrews, "Spectrum Allocation in Tiered Cellular Networks," IEEE Transaction on Communication, vol. 57, pp. 3059 - 3068, October 2009.

[11] D. Lopez and J. Zhang, "OFDMA Femtocells: A Self-Organizing Approach for Frequency Assignment," IEEE International Symposium on Personal, Indoor and Mobile Radio Communications, pp. 2202 - 2207, April 2010.

[12] J. Wang and W. Ching, "The Analysis of System Performance of An MC-CDMA System over Two-Tier Femtocell Networks," IEEE International Multiconference of Engineers and Computer Scientists, vol. 2, pp. 864-867, March 2010.

[13] C. Chang, "An Interference-Avoidance Code Assignment Strategy for the Hierarchical Two-Dimensional-Spread MC-DS-CDMA System: A Prototype of Cognitive Radio Femtocell System ," IEEE Vehicular Technology Conference, vol. 61, pp. 166 - 184, Janauary 2012.

[14] W. Cheng and Y. Li, "Energy Efficient Spectrum Allocation for Green Radio in Two-tier Cellular Networks," Global Telecommunication Conference, pp. 1-5, December 2010.

[15] F. Richter and G. Fettweis, "Energy Efficiency Aspects of Base Station Deployment Strategies for Cellular Networks," IEEE Vehicular Technology Conference, pp. 1-5, Janauary 2010. 
[16] R. Kim and K. Etemad, "WiMAX Femtocell: Requirements, Challenges and Solutions," IEEE Communication Magazine, vol. 47, pp. 84-91, September 2009.

[17] L. Wang and Y. Zhang, "Mobility Management Schemes at Radio Network Layer for LTE Femtocells," IEEE Vehicular Technology Conference, pp. 1-5, April 2009.

[18] H. Schulze and C. Luders, Theory and Applications of OFDM and CDMA. John Wiley and Sons, 2005.

[19] L. Hanzo, M. Munster, B. J. Choi, and T. Keller, OFDM and MC-CDMA for Broadband Multi-User Communications, WLANs and Broadcasting. John Wiley and Sons Ltd., West Sussex, England, 2003.

[20] Q.Chen and E.Sousa, "Multicarrier CDMA with Adaptive Frequency Hopping for Mobile Radio Systems," IEEE Vehicular Technology Conference, vol. 14, pp. 1852 - 1858, December 1996.

[21] L. Khalid and A. Anpalagan, "Performance Analysis of a Threshold-Based GroupAdaptive Modulation Scheme with Adaptive Subcarrier Allocation in OFCDM Systems," IEEE Transaction on Wireless Communication, vol. 7, pp. 2463 - 2467, July 2008.

[22] R. Prasad and S. Hara, "Overview of Multicarrier CDMA," IEEE Communications Magazine, pp. 126-133, December 1977.

[23] A. Persson and E. Strom, "Time-Frequency Localized CDMA for Downlink Multi-carrier Systems," in IEEE International Symposium on Spread Spectrum Techniques and Applications, vol. 1, pp. 118-122, September 2002.

[24] N. Maeda, Y. Kishiyama, H. Atarashi, and M. Sawashashi, "Variable Spreading FactorOFCDM with Two Dimensional Spreading that Prioritizes Time Domain Spreading for 
Forward Link Broadband Wireless Access," in IEEE Vehicular Technology Conference, vol. 1, pp. 127-132, April 2003.

[25] A. Matsumoto, K. Miyoshi, M. Uesugi, and O. Kato, "A Study on Time Domain Spreading for OFCDM," in IEEE International Symposium on Wireless Personal Multimedia Communications, vol. 2, pp. 725-728, October 2002.

[26] R. Caldwell and A. Anpalagan, "Adaptive Subcarrier Allocation in Synchronous Reverse Links of a Multicarrier CDMA System with Time and Frequency Spreading ," IEEE Transaction on Vehicular Technology, vol. 57, pp. 1494 - 1501, May 2008.

[27] K. Kitagawa and O. Kato, "An OFDM-CDMA System using Combination of Time and Frequency Domain Spreading," Tech. Rep. RCS2000-3, Institute of Electronics, Information and Communication Engineers Technical Report, 2000.

[28] C. You and D. Hong, "Multicarrier CDMA Systems Using Time-Domain and FrequencyDomain Spreading Codes," IEEE Transactions on Communications, vol. 51, pp. 17-21, January 2003.

[29] D. Lopez and G. Roche, "Access Methods to WiMAX FemtoCells: A Downlink System-Level Case Study," IEEE International Conference on Communication Systems, pp. 1657 - 1662, November 2008.

[30] J. Chih-Lin and R.Gitlin, "A Microcell/Macrocell Cellular Architecture for Low- and High-Mobility Wireless Users," IEEE Journal on Selected Areas in Communication, vol. 11, pp. 885-891, August 2002.

[31] F.Dooley and Y. Ji, "Dynamic Fractional Frequency Reuse based Hybrid Resource Management for Femtocell Networks," IEEE Wireless Communications and Mobile Computing Conference, pp. 272 - 277, July 2011. 
[32] M. C. Necker, "Integrated Scheduling and Interference Coordination in Cellular OFDMA Networks," IEEE Conference on Broadband Communications, Networks and Systems, pp. 559-566, September 2007.

[33] F. Pantisano and M. Bennis, "Interference Avoidance via Resource Scheduling in TDD Underlay Femtocells," IEEE International Symposium on Personal, Indoor and Mobile Radio Communications, pp. 175-179, December 2010.

[34] D. Lopez and J. Zhang, "Limited Access to OFDMA Femtocells," IEEE International Symposium on Personal, Indoor and Mobile Radio Communications, pp. 1-5, September 2009.

[35] V. Chandrasekhar and J. G. Andrews, "Open vs. Closed Access Femtocells in the Uplink," IEEE Transaction on Wireless Communication, vol. 9, pp. 2463-2467, December 2010.

[36] M. EI-Gendy and E. Sourour, "A Study of Access Methods Effect on the Performance of Two-Tier LTE Femtocell Networks," IEEE National Radio Science Conference, pp. 1-8, April 2011.

[37] S.Weber and J. Andrews, "Transmission Capacity of Wireless Ad Hoc Networks with Successive Interference Cancellation," IEEE Transaction on Information Theory, vol. 51, pp. 4091-4102, August 2005.

[38] H. Claussen, "Femtocell Coverage Optimization using Switched Multielement Antennas," IEEE International Conference on Communication, pp. 1-6, June 2009.

[39] D. Lopez and A. Valcarce, "OFDMA Femtocells: A Roadmap on Interference Avoidance," IEEE Communication Magazine, pp. 41-48, September 2009. 
[40] J.Haitham and M.Salleh, "Multi-carrier Transmission Techniques for Wireless Communication Systems: A Survey," World Scientific and Engineering Academy and Society Transaction on Communications, vol. 8, May 2009.

[41] Y. Lee and Y. Chung, "Interference Analysis for Femtocell Deployment in OFDMA Systems Based on Fractional Frequency Reuse," IEEE International Conference on Communication Circuits and Systems, vol. 15, pp. 425-427, April 2011.

[42] G. Cao and X. Zhang, "An Adaptive Sub-band Allocation Scheme for Dense Femtocell Environment," IEEE Wireless Communications and Networking Conference, pp. 102107, August 2011.

[43] C. Crespo and R. Agust, "Self-optimization of Spectrum Assignment and Transmission Power in OFDMA Femtocells," IEEE Transaction on Communication, pp. 404 - 409, September 2010.

[44] S. Das and P. Chandhar, "Issues in Femtocell Deployment in Broadband OFDMA Networks:3GPPLTE a Case Study," IEEE Vehicular Technology Conference, pp. 1-5, December 2011.

[45] V. Chandrasekhar and J. Andrews, "Uplink Capacity and Interference Avoidance for Two-Tier Cellular Networks," IEEE Global Telecommunications Conference, pp. 3322 - 3326, November 2007.

[46] L. Bao and D. Kim, "Distributed Interference Management in Femtocell Networks," IEEE Vehicular Technology Conference, pp. 1-5, September 2011.

[47] G. Joshi and C. Patel, "Performance Analysis of Active Handoff in CDMA2000 Femtocells," National Communication Conference, pp. 1-5, Janauary 2010.

[48] K. Fazel and S. Kaiser, Multi-carrier Spread Spectrum and Related Topics. Kluwer Academic Publishers, Boston, USA, 1999. 
[49] I. Guvenc and U. Kozat, "Impact of Spreading on the Capacity of Neighboring Femtocells ," IEEE International Symposium on Personal, Indoor and Mobile Radio Communications, pp. 1814 - 1818, September 2009.

[50] S. M. Zafi and A. A. Memon, "Performance Comparison of OFDM, MC-CDMA and OFCDM for 4G Wireless Broadband Access and Beyond," Progress in Electromagnetics Research Symposium Proceedings, March 2011.

[51] A. Atarashi and Sawahashi, "Variable Spreading Factor Orthogonal Frequency and Code Division Multiplexing (VSF-OFCDM) for Broadband Packet Wireless Access," IEEE Vehicular Technology Conference, vol. 1, pp. 127-132, 2003.

[52] K. Zheng and W. Wang, "Performance Analysis for OFDM-CDMA with Joint Frequency-Time Spreading," IEEE Transaction on Broadcasting, vol. 51, pp. 144-148, March 2005. 